\title{
Synergistic Copper-Catalyzed Reductive Aminocarbonylation of Alkyl Iodides with Nitroarenes
}

\author{
Siling Zhao and Neal P. Mankad* \\ Department of Chemistry, University of Illinois at Chicago, 845 W. Taylor Street, Chicago, Illinois \\ *npm@uic.edu
}

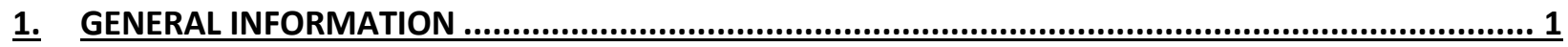

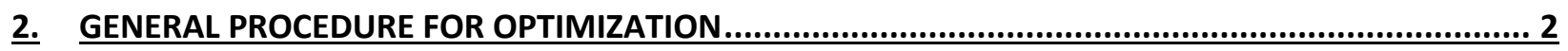

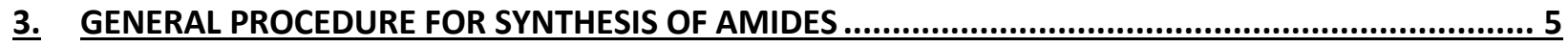

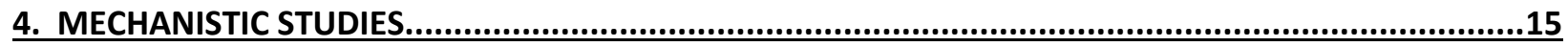

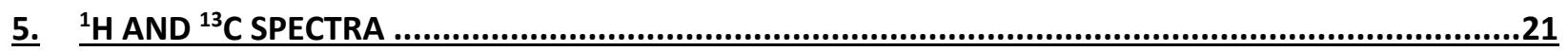

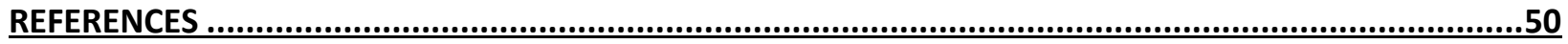




\section{General Information}

General Procedures. Reactions requiring anhydrous conditions were conducted in a $\mathrm{N}_{2}$-filled glovebox or using standard Schlenk line techniques. Reactions at greater than atmospheric pressure were conducted in a Parr 4621 General Purpose Pressure Reactor. A fitted, aluminum insert was custom-made for the Parr reactor that could hold up to nine 22-mL scintillation vials for running reactions in parallel. Thin layer chromatography (TLC) was conducted with E. Merck silica gel 60 F254 pre-coated plates $(0.25 \mathrm{~mm})$ and visualized by UV light $(254 \mathrm{~nm})$ or $\mathrm{KMnO}_{4}$ stain. Purification of compounds was achieved by column chromatography using Merck Flash Silica Gel 60 (230-400 mesh). Organic solutions were concentrated under reduced pressure using a rotary evaporator.

Materials. Deuterated solvents were purchased from Cambridge Isotope Laboratories, Inc. Common commercial reagents were purchased from Sigma-Aldrich, Fisher Scientific or VWR International Co. and used without further purification unless otherwise noted. Solvents were dried using a Glass Contour Solvent System built by Pure Process Technology, LLC. CO gas was purchased from Praxair at a purity of $99.99 \%$ (4.0RS research grade) and used directly from the cylinder. $\mathrm{IPrCuCl}^{1}, \mathrm{SIPrCuCl}^{2}{ }^{2} \mathrm{IMesCuCl},{ }^{1} \mathrm{SIMesCuCl}^{2},{ }^{\mathrm{Me}} \mathrm{IPrCuCl}^{3},{ }^{\mathrm{Br}} \mathrm{IMesCuCl},{ }^{3}$ ${ }^{\mathrm{Cl}} \mathrm{IPrCuCl},{ }^{3}$ and ${ }^{\mathrm{Cl}}{ }_{\mathrm{OMeIMes}} \mathrm{IMCl}^{3}$ were prepared according to literature procedures. Alkyl iodides were prepared from corresponding alcohols according to literature. ${ }^{4}$

Instrumentation. Nuclear Magnetic Resonance (NMR) spectra were recorded on BRUKER AV (400 $\mathrm{MHz})$ or BRUKER AV $(500 \mathrm{MHz})$ spectrometers at $298 \mathrm{~K}$. Chemical shifts $(\delta)$ are reported in ppm with the residual solvent signal as internal standard (chloroform at 7.26 and $77.16 \mathrm{ppm}$ for ${ }^{1} \mathrm{H}$ and ${ }^{13} \mathrm{C}$ NMR spectroscopy, respectively). Abbreviations for signal coupling are as follows: s, singlet; d, doublet; t, triplet; q, quartet; quin., quintet; $\mathrm{m}$, multiplet; br., broad. Coupling constants were taken from the spectra directly and are uncorrected. ${ }^{1} \mathrm{H}$ and ${ }^{13} \mathrm{C}$ NMR data provided are taken directly using material for which the yield is quoted, without further purification, and are representative of purity. FT-IR spectra were recorded on a Bruker Platinum ATR spectrometer. Absorptions are given in wavenumbers $\left(\mathrm{cm}^{-1}\right)$. HRMS (ESI) were measured with a Waters Q-TOF Ultima ESI Mass Spectrometer. 


\section{General Procedure for optimization}

In a glovebox, base, copper catalyst, and. 1,4-dioxane $(2.0 \mathrm{~mL})$ were added to a $20-\mathrm{mL}$ vial charged with a 1.5 $\mathrm{cm}$ stir bar. Then nitrobenzene $(0.1 \mathrm{mmol}, 1.0 \mathrm{eq}), 3$-iodo-1-phenylpropane $(0.2 \mathrm{mmol}, 2.0 \mathrm{eq})$ and hydrosilane were added to the mixture. The vial was then placed in an aluminum rack within a Parr pressure reactor. Next, the reactor was closed and taken out of the glovebox. A CO gas cylinder with a pressure regulator was connected to the reactor. The reactor was pressurized with $\mathrm{CO}$ gas and released after $5 \mathrm{~min}$. This procedure was repeated three times, after which the reaction was heated on heating mantle and stirred vigorously. After desired reaction time, the reaction was cooled to room temperature and the $\mathrm{CO}$ gas was released. The solvent was removed under reduced pressure. The residue was diluted with $\mathrm{Et}_{2} \mathrm{O}$ and then filtered through a pad of silica gel (a pipette with about $5 \mathrm{~cm}$ silica gel). The filtrate was concentrated under reduced pressure. The residue was dissolved in $\mathrm{CDCl}_{3}$, and $\mathrm{CHCl}_{2} \mathrm{CHCl}_{2}(0.1 \mathrm{mmol})$ was added as internal standard for ${ }^{1} \mathrm{H}$ NMR analysis.

\section{Table S1. Base Investigation}

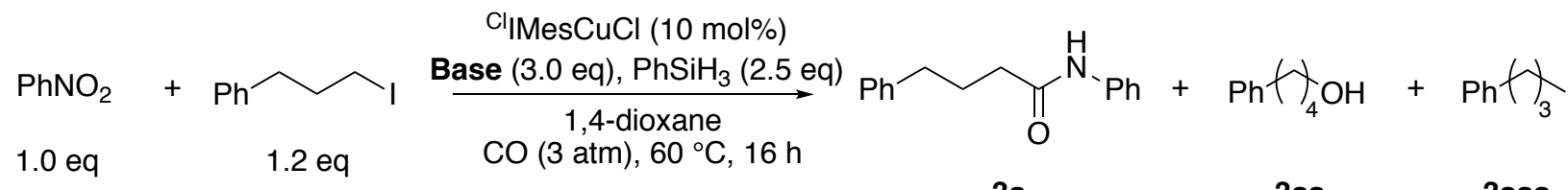

\begin{tabular}{cccc}
\hline Base & Yield of 3a (\%) & Yield of 3aa (\%) & Yield of 3aaa (\%) \\
\hline $\mathrm{KF}$ & 0 & 0 & 0 \\
$\mathrm{KOH}$ & 0 & 20 & 0 \\
$\mathrm{NaOH}$ & 28 & 30 & 0 \\
$\mathrm{LiOMe}$ & 24 & 22 & 11 \\
$\mathrm{KOMe}$ & 0 & 19 & 0 \\
$\mathrm{CsF}$ & 16 & 20 & 0 \\
$\mathrm{CsOAc}$ & 21 & 22 & 0 \\
\hline
\end{tabular}

Table S2. Silane Investigation

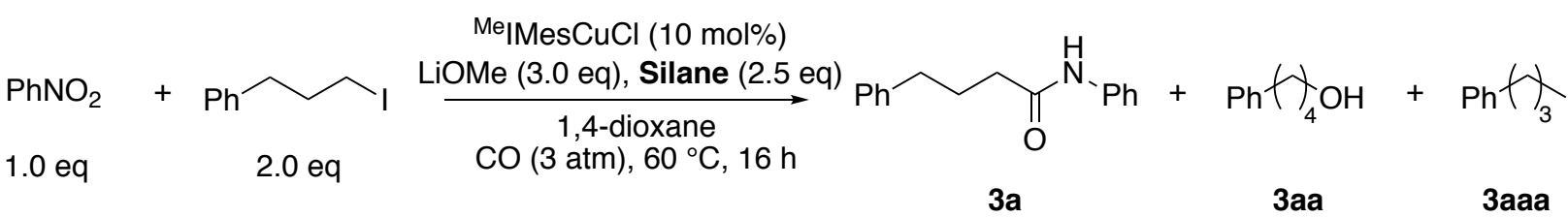

\begin{tabular}{cccc}
\hline Silane & Yield of 3a (\%) & Yield of 3aa (\%) & Yield of 3aaa (\%) \\
\hline $\mathrm{PhSiH}_{3}$ & 10 & 0 & 0 \\
$\mathrm{Et}_{3} \mathrm{SiH}$ & 0 & 0 & 0 \\
$(\mathrm{EtO})_{3} \mathrm{SiH}$ & 0 & 0 & 0 \\
$\mathrm{PMHS}$ & 0 & 0 & 0 \\
\hline
\end{tabular}

PMHS = (Poly)methylhydrosiloxane.

\section{Table S3. Catalyst Investigation}




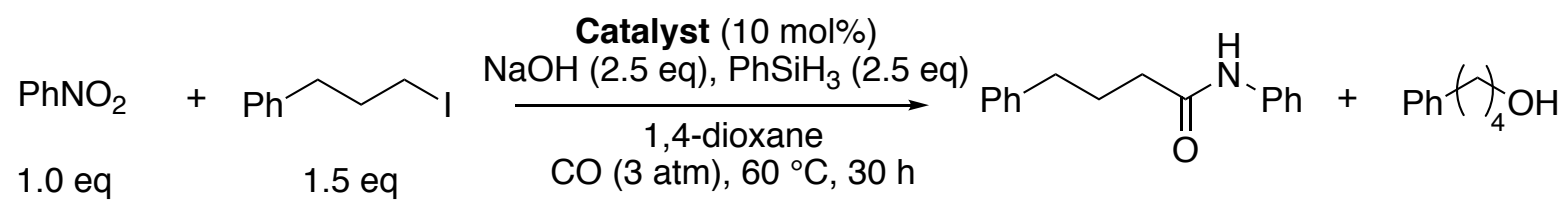

3a 3aa

\begin{tabular}{ccc}
\hline Catalyst & Yield of 3a (\%) & Yield of 3aa (\%) \\
\hline${ }^{\mathrm{Cl}} \mathrm{IMesCuCl}$ & 31 & 0 \\
$\mathrm{IMesCuCl}$ & 32 & 20 \\
$\mathrm{SIMesCuCl}$ & 20 & 0 \\
${ }^{\mathrm{Me}} \mathrm{IMesCuCl}$ & 22 & 14 \\
${ }^{\mathrm{Cl}} \mathrm{IPrCuCl}$ & 10 & 26 \\
$\mathrm{IPrCuCl}$ & 5 & 22 \\
$\mathrm{SIPrCuCl}$ & 13 & 26 \\
${ }_{\mathrm{Me}} \mathrm{IPrCuCl}$ & 8 & 36 \\
\hline
\end{tabular}<smiles>[R]N1CN([R])C(C)=C1C</smiles>

MejPr

MeIMes

$$
R=2,6 \text {-(diisopropyl)phenyl }
$$

$R=$ 2,4,6-trimethylphenyl $\begin{array}{ll}\text { SIPr } & R=2,6 \text {-(diisopropyl)phenyl } \\ \text { SIMes } & R=2,4,6 \text {-trimethylphenyl }\end{array}$

Table S4. Amount of Base Investigation

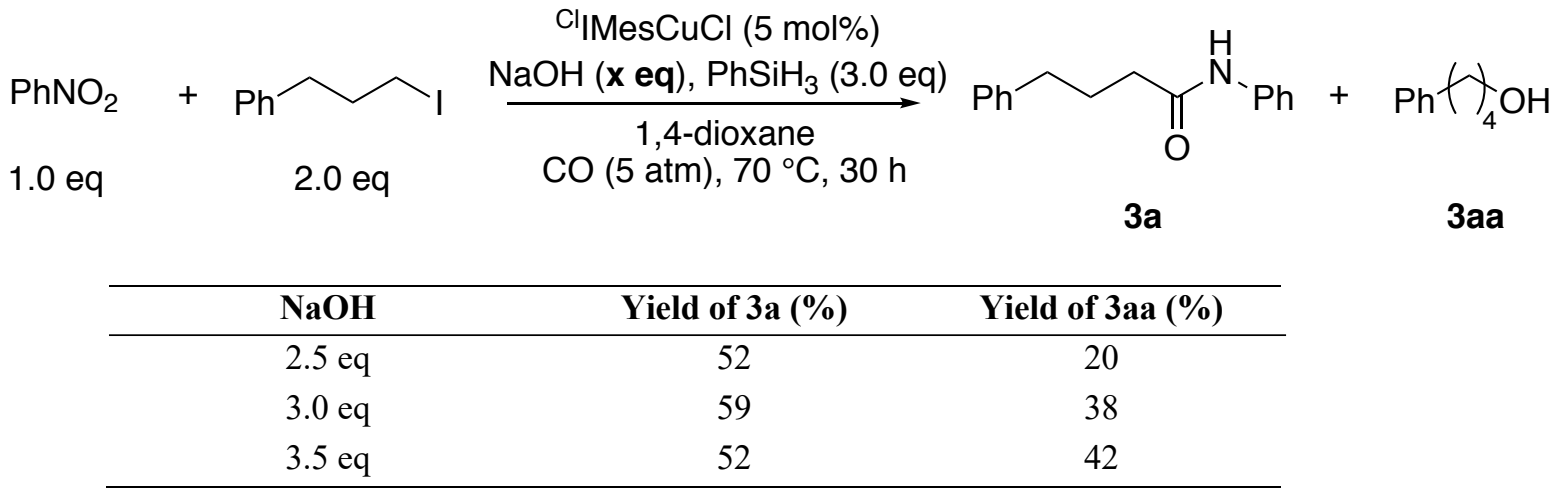

Table S5. Amount of Silane Investigation

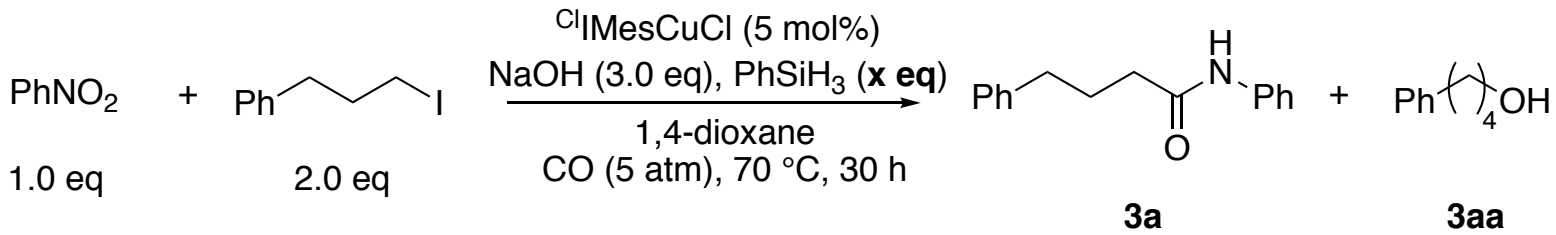




\begin{tabular}{ccc}
\hline $\mathbf{P h S i H}_{\mathbf{3}}$ & Yield of 3a (\%) & Yield of 3aa (\%) \\
\hline $2.5 \mathrm{eq}$ & 54 & 30 \\
$3.0 \mathrm{eq}$ & 59 & 38 \\
$3.5 \mathrm{eq}$ & 53 & 45 \\
\hline
\end{tabular}

Table S6. Amount of Alkyl Iodides Investigation

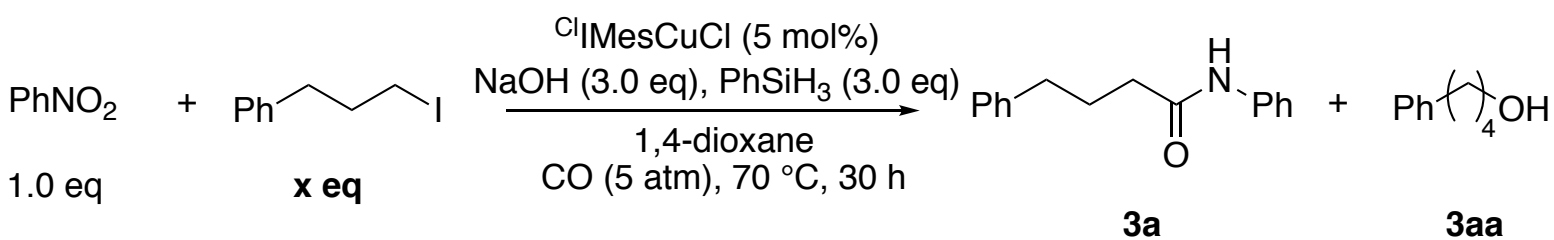

\begin{tabular}{ccc}
\hline 3-Iodo-1-phenylpropane & Yield of 3a (\%) & Yield of 3aa (\%) \\
\hline 0.67 eq & 0 & 0 \\
$1.2 \mathrm{eq}$ & 28 & 30 \\
$1.5 \mathrm{eq}$ & 49 & 41 \\
$2.0 \mathrm{eq}$ & 59 & 32 \\
\hline
\end{tabular}

Table S7. Pressure Investigation

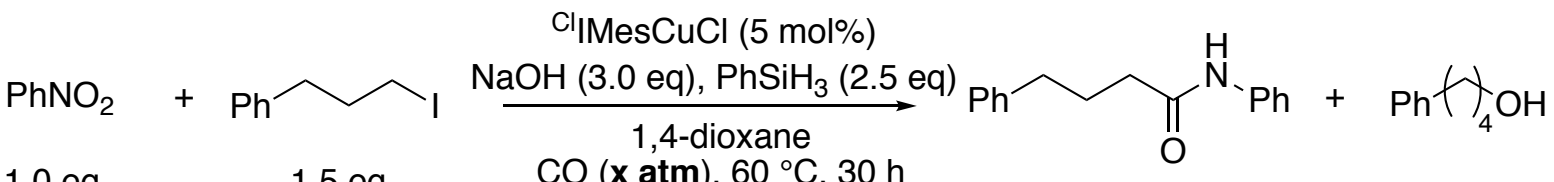

$1.0 \mathrm{eq} \quad 1.5 \mathrm{eq} \quad \mathrm{CO}(\mathbf{x ~ a t m}), 60^{\circ} \mathrm{C}, 30 \mathrm{~h}$

$3 a$

3aa

\begin{tabular}{ccc}
\hline CO & Yield of 3a (\%) & Yield of 3aa (\%) \\
\hline 3 atm & 30 & 18 \\
5 atm & 50 & 44 \\
6 atm & 42 & 40
\end{tabular}

Table S8. Temperature Investigation

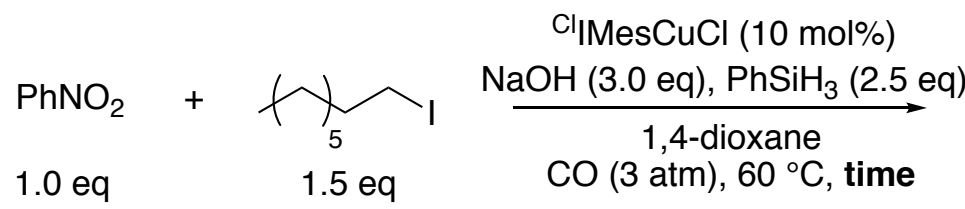<smiles>CCC(C)(C)CC(=O)Nc1ccccc1</smiles>

30

\begin{tabular}{cc}
\hline T $\left({ }^{\circ} \mathbf{C}\right)$ & Yield of 3o (\%) \\
\hline 50 & 0 \\
60 & 48
\end{tabular}




\begin{tabular}{ll}
\hline 70 & 62 \\
80 & 54 \\
\hline
\end{tabular}

Table S9. Catalyst Loading Investigation

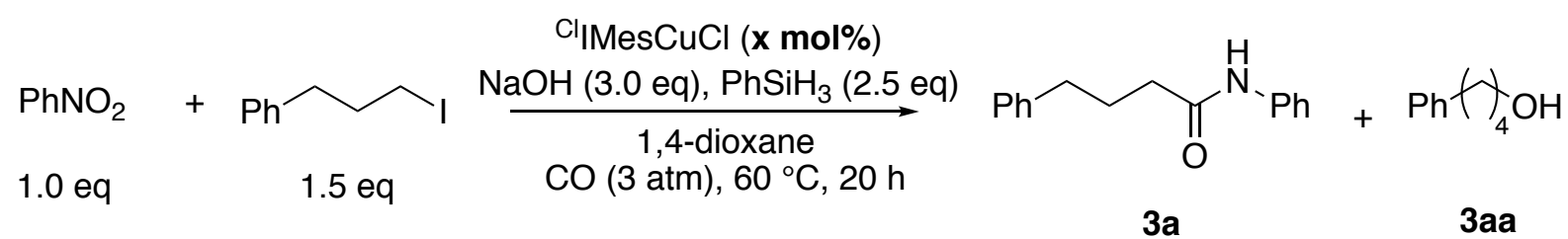

\begin{tabular}{ccc}
\hline Catalyst Loading (mol\%) & Yield of 3a (\%) & Yield of 3aa (\%) \\
\hline 5 & 47 & 26 \\
8 & 26 & 20 \\
10 & 23 & 20 \\
12 & 15 & 18 \\
\hline
\end{tabular}

Table S10. Reaction Time Investigation

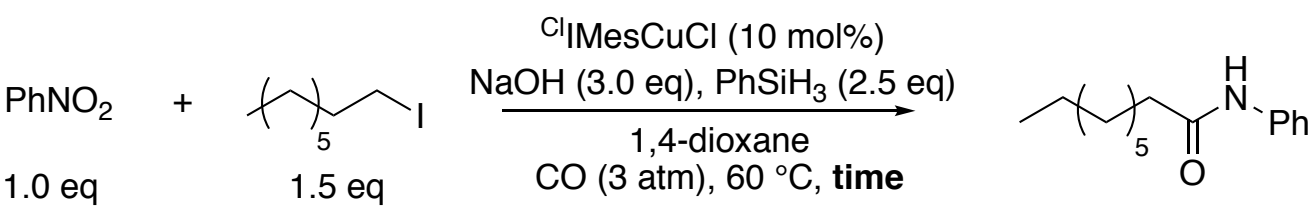

30

\begin{tabular}{cc}
\hline Time (h) & Yield of 3o (\%) \\
16 & 26 \\
20 & 35 \\
24 & 48 \\
30 & 52 \\
\hline
\end{tabular}

\section{General Procedure for Synthesis of Amides}

In a glovebox, $\mathrm{NaOH}(0.012 \mathrm{~g}, 3.0 \mathrm{mmol}, 3.0 \mathrm{eq})$, catalyst $(0.05 \mathrm{mmol}, 5 \mathrm{~mol} \%)$, and 1,4-dioxane $(2.0 \mathrm{~mL})$ were added to a $20-\mathrm{mL}$ vial charged with a $1.5-\mathrm{cm}$ stir bar. Then nitroarene $(0.1 \mathrm{mmol}, 1.0 \mathrm{eq})$, alkyl iodide $(0.2$ mmol, 2.0 eq) and phenylsilane ( $37 \mu \mathrm{L}, 3.0 \mathrm{mmol}, 3.0 \mathrm{eq})$ were added to the mixture. Then the vial was placed in an aluminum rack within a Parr pressure reactor. Next, the reactor was closed and taken out of the glovebox. A CO gas cylinder with a pressure regulator was connected to the reactor. The reactor was pressurized with CO gas and released after $5 \mathrm{~min}$. This procedure was repeated three times, after which the reaction was heated to $70{ }^{\circ} \mathrm{C}$ and stirred vigorously. After $30 \mathrm{~h}$, the reaction was cooled to room temperature and the $\mathrm{CO}$ gas was released. The solvent was removed under reduced pressure. The residue was dissolved with $\mathrm{Et}_{2} \mathrm{O}$, then filtered through a pad of silica gel (a pipette with about $5 \mathrm{~cm}$ silica gel). The filtrate was concentrated under reduced pressure for silica column chromatography. 
<smiles>O=C(CCCc1ccccc1)Nc1ccccc1</smiles>

$N$,4-diphenylbutanamide (3a) Prepared according to general procedure using $1 \mathrm{a}(10 \mu \mathrm{L}, 0.1 \mathrm{mmol})$ and $\mathbf{2 a}$ ( $32 \mu \mathrm{L}, 0.2 \mathrm{mmol})$. The crude materials were combined from two parallel experiments and were purified by silica gel chromatography (hexane/ethyl acetate $=90: 10, \mathrm{Rf}=0.18)$ to afford the title compound as a white solid $(0.037 \mathrm{~g}, 78 \%)$. Characterization has been reported by previous literature. ${ }^{5} 1 \mathrm{mmol}$ scale reaction was done according to the above general procedure and separated using the same elute on silica gel chromatography. $0.26 \mathrm{~g}$ white solid was obtained from two parallel experiments in the yield of $54 \%$.

${ }^{1} \mathbf{H}$ NMR (500 MHz, $\left.\mathbf{C D C l}_{3}\right) \delta 7.49(\mathrm{~d}, J=7.9 \mathrm{~Hz}, 2 \mathrm{H}), 7.33-7.28(4 \mathrm{H}), 7.21-7.20(\mathrm{~m}, 3 \mathrm{H}), 7.12-7.08(\mathrm{~m}$, $1 \mathrm{H}), 7.10(\mathrm{~s}, 1 \mathrm{H}), 2.73(\mathrm{t}, J=7.3 \mathrm{~Hz}, 2 \mathrm{H}), 2.35(\mathrm{t}, J=7.5 \mathrm{~Hz}, 2 \mathrm{H}), 2.11-2.05(\mathrm{~m}, 2 \mathrm{H})$.

${ }^{13} \mathrm{C}$ NMR (126 MHz, $\left.\mathbf{C D C l}_{3}\right)$ 171.0, 129.2, 128.7, 128.6, 126.2, 124.4, 119.9, 36.9, 35.2, 27.0

HRMS (ESI) Calcd. for $\mathrm{C}_{15} \mathrm{H}_{23} \mathrm{NO}\left([\mathrm{M}+\mathrm{H}]^{+}\right)$: 234.1858; Found: 234.1864 .

IR (neat) 3267, 3024, 2922, 1663, 1598, 1520, 1443, 1376, 1331, 1250, 1193, 731, 686, $497 \mathrm{~cm}^{-1}$.

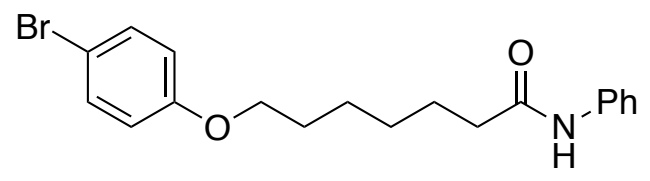

7-(4-bromophenoxy)-N-phenylheptanamide (3b) Prepared according to general procedure using 1a (10 $\mu \mathrm{L}, 0.1 \mathrm{mmol})$ and $\mathbf{2 b}(0.076 \mathrm{~g}, 0.2 \mathrm{mmol})$. The crude materials were combined from two parallel experiments and were purified by silica gel chromatography (hexane/ethyl acetate $=80: 20, \mathrm{Rf}=0.22$ ) to afford the title compound as a white solid $(0.054 \mathrm{~g}, 72 \%)$.

${ }^{1} \mathbf{H}$ NMR (500 MHz, CDCl $) \delta 7.51(\mathrm{~d}, J=7.9 \mathrm{~Hz}, 2 \mathrm{H}), 7.36-7.34(\mathrm{~m}, 2 \mathrm{H}), 7.33-7.30(\mathrm{~m}, 2 \mathrm{H}), 7.14-7.09$ $(\mathrm{m}, 2 \mathrm{H}), 6.76(\mathrm{~d}, J=9.0 \mathrm{~Hz}, 2 \mathrm{H}), 3.91(\mathrm{t}, J=6.4 \mathrm{~Hz}, 2 \mathrm{H}), 2.37(\mathrm{t}, J=7.4 \mathrm{~Hz}, 2 \mathrm{H}), 1.81-1.74(\mathrm{~m}, 4 \mathrm{H}), 1.53-1.42(\mathrm{~m}$, $4 \mathrm{H})$.

${ }^{13}$ C NMR (126 MHz, $\left.\mathbf{C D C l}_{3}\right) \delta 171.2,158.3,138.0,132.3,129.2,124.4,119.9,116.4,112.8,68.2,37.8$, 29.13, 29.07, 26.0, 25.6.

HRMS (ESI) Calcd. for $\mathrm{C}_{19} \mathrm{H}_{23} \mathrm{BrNO}_{2}\left([\mathrm{M}+\mathrm{H}]^{+}\right)$: 376.0912; Found: 376.0908 .

IR (neat) 3300, 2940, 2881, 1660, 1597, 1531, 1488, 1442, 1214, 818, 691, $500 \mathrm{~cm}^{-1}$.<smiles>O=C(CCCCCCOc1ccc(I)cc1)Nc1ccccc1</smiles>

7-(4-iodophenoxy)- $N$-phenylheptanamide (3c) Prepared according to general procedure using $1 \mathrm{a}(10 \mu \mathrm{L}$, $0.1 \mathrm{mmol})$ and $2 \mathrm{c}(0.086 \mathrm{~g}, 0.2 \mathrm{mmol})$. The crude materials were combined from two parallel experiments and were purified by silica gel chromatography (hexane/ethyl acetate $=80: 20, \mathrm{Rf}=0.20$ ) to afford the title compound as a white solid $(0.049 \mathrm{~g}, 58 \%)$.

${ }^{1}$ H NMR (500 MHz, CDCl $) \delta$ 7.56-7.48 (m, 4H), $7.32(\mathrm{t}, J=7.3 \mathrm{~Hz}, 2 \mathrm{H}), 7.13-7.07$ (m, 2H), 6.66 (d, $J=$ $8.9 \mathrm{~Hz}, 2 \mathrm{H}), 3.91(\mathrm{t}, J=6.4 \mathrm{~Hz}, 2 \mathrm{H}), 2.37(\mathrm{t}, J=7.4 \mathrm{~Hz}, 2 \mathrm{H}), 1.81-1.74(\mathrm{~m}, 4 \mathrm{H}), 1.52-1.42(\mathrm{~m}, 4 \mathrm{H})$.

${ }^{13}$ C NMR (126 MHz, $\left.\mathbf{C D C l}_{3}\right) \delta 171.2,159.0,138.3,129.2,119.9,117.1,82.6,68.0,37.8,29.1,29.1,26.0$, 25.6.

HRMS (ESI) Calcd. for $\mathrm{C}_{19} \mathrm{H}_{23} \mathrm{INO}_{2}\left([\mathrm{M}+\mathrm{H}]^{+}\right)$: 424.0774; Found: 424.0768.

IR (neat) 3307, 2939, 2854, 1651, 1589, 1531, 1486, 1435, 1283, 1247, 1176, 753, $500 \mathrm{~cm}^{-1}$. 
<smiles>O=C(CCCCCCOc1ccc(Cl)cc1)Nc1ccccc1</smiles>

7-(4-chlorophenoxy)- $N$-phenylheptanamide (3d) Prepared according to general procedure using $1 a(10 \mu \mathrm{L}$, $0.1 \mathrm{mmol})$ and $2 \mathrm{~d}(0.067 \mathrm{~g}, 0.2 \mathrm{mmol})$. The crude materials were combined from two parallel experiments and were purified by silica gel chromatography (hexane/ethyl acetate $=80: 20, \mathrm{Rf}=0.21$ ) to afford the title compound as a white solid $(0.046 \mathrm{~g}, 70 \%)$.

${ }^{1}$ H NMR (500 MHz, $\left.\mathbf{C D C l}_{3}\right) \delta 7.51(\mathrm{~d}, J=7.9 \mathrm{~Hz}, 2 \mathrm{H}), 7.32(\mathrm{t}, J=7.6 \mathrm{~Hz}, 2 \mathrm{H}), 7.22-7.20(\mathrm{~m}, 2 \mathrm{H}), 7.17(\mathrm{~s}$, $1 \mathrm{H}), 7.11-7.08(\mathrm{t}, J=7.5 \mathrm{~Hz}, 1 \mathrm{H}),, 6.82-6.79(\mathrm{~d}, J=9.0 \mathrm{~Hz}, 2 \mathrm{H}), 3.91(\mathrm{t}, J=6.4 \mathrm{~Hz}, 2 \mathrm{H}), 2.37(\mathrm{t}, J=7.5 \mathrm{~Hz}, 2 \mathrm{H})$, 1.77 (sextet, $J=7.2 \mathrm{~Hz}, 4 \mathrm{H}), 1.52-1.43(\mathrm{~m}, 4 \mathrm{H})$.

${ }^{13} \mathbf{C}$ NMR (126 MHz, $\left.\mathbf{C D C l}_{3}\right) \delta 171.3,157.8,138.0,129.4,129.2,125.5,124.4,119.9,115.9,77.2,68.2$, $37.8,29.2,29.1,26.0,25.6$.

HRMS (ESI) Calcd. for $\mathrm{C}_{19} \mathrm{H}_{23} \mathrm{NO}_{2} \mathrm{Cl}\left([\mathrm{M}+\mathrm{H}]^{+}\right)$: 332.1417; Found: 332.1420 .

$\mathrm{cm}^{-1}$.

IR (neat) 3292.66, 2935.09, 2855.30, 1591.02, 1490.30, 1471.96, 1200.63, 1173.93, 1073.14, 996.69, 829.83<smiles>O=C(CCCCCCOCCCc1ccccc1)Nc1ccccc1</smiles>

$N$-phenyl-7-(3-phenylpropoxy)heptanamide (3e) Prepared according to general procedure using $1 a(10 \mu \mathrm{L}$, $0.1 \mathrm{mmol})$ and $2 \mathrm{e}(0.069 \mathrm{~g}, 0.2 \mathrm{mmol})$. The crude materials were combined from two parallel experiments and were purified by silica gel chromatography (hexane/ethyl acetate $=80: 20, \mathrm{Rf}=0.18$ ) to afford the title compound as a colorless liquid $(0.051 \mathrm{~g}, 75 \%)$.

${ }^{1}$ H NMR (500 MHz, CDCl $) \delta 7.51(\mathrm{~d}, J=7.9 \mathrm{~Hz}, 2 \mathrm{H}), 7.30(\mathrm{~m}, 5 \mathrm{H}), 7.19(\mathrm{~d}, J=7.5 \mathrm{~Hz}, 3 \mathrm{H}), 7.10(\mathrm{t}, J=$ $9.5 \mathrm{~Hz}, 1 \mathrm{H}), 3.41(\mathrm{td}, J=6.5,3.8 \mathrm{~Hz}, 4 \mathrm{H}), 2.69(\mathrm{t}, J=7.7 \mathrm{~Hz}, 2 \mathrm{H}), 2.35(\mathrm{t}, J=7.5 \mathrm{~Hz}, 2 \mathrm{H}), 1.92-1.88(\mathrm{~m}, 2 \mathrm{H}), 1.76-$ $1.72(\mathrm{~m}, 2 \mathrm{H}), 1.60(\mathrm{t}, J=6.5 \mathrm{~Hz}, 2 \mathrm{H}), 1.42(\mathrm{~d}, J=6.8 \mathrm{~Hz}, 4 \mathrm{H})$.

${ }^{13} \mathbf{C}$ NMR (126 MHz, $\left.\mathbf{C D C l}_{3}\right) \delta 171.4,142.2,138.1,129.1,128.6,128.4,125.9,124.3,119.9,70.9,70.1$, $37.9,32.5,31.5,29.7,29.2,26.1,25.7$.

HRMS (ESI) Calcd. for $\mathrm{C}_{22} \mathrm{H}_{30} \mathrm{NO}_{2}\left([\mathrm{M}+\mathrm{H}]^{+}\right)$: 340.2277; Found: 340.2283 .

IR (neat) 2919, 2850, 1585, 1487, 1316, 1199, 1152, 1066, 841, $492 \mathrm{~cm}^{-1}$.<smiles>O=C(CCCCCCOCc1ccccc1)Nc1ccccc1</smiles>

7-(benzyloxy)-N-phenylheptanamide (3f) Prepared according to general procedure using 1a (10 $\mu \mathrm{L}, 0.1$ $\mathrm{mmol})$ and $\mathbf{2 f}(0.064 \mathrm{~g}, 0.2 \mathrm{mmol})$. The crude materials were combined from two parallel experiments and were purified by silica gel chromatography (hexane/ethyl acetate $=80: 20, \mathrm{Rf}=0.20$ ) to afford the title compound as a light yellow solid $(0.046 \mathrm{~g}, 74 \%)$.

${ }^{1} \mathbf{H}$ NMR (500 MHz, CDCl $) \delta 7.51(\mathrm{~s}, 2 \mathrm{H}), 7.34-7.26(\mathrm{~m}, 6 \mathrm{H}), 7.18(\mathrm{~s}, 1 \mathrm{H}), 7.11-7.08(\mathrm{t}, J=7.5 \mathrm{~Hz}, 1 \mathrm{H})$, $4.50(\mathrm{~s}, 2 \mathrm{H}), 3.47$ (t, $J=6.2 \mathrm{~Hz}, 2 \mathrm{H}), 2.34(\mathrm{t}, J=7.5 \mathrm{~Hz}, 2 \mathrm{H}), 1.73(\mathrm{q},, J=7.0 \mathrm{~Hz}, 2 \mathrm{H}), 1.62(\mathrm{q}, J=10.0 \mathrm{~Hz}, 2 \mathrm{H})$, $1.41(\mathrm{~m}, 4 \mathrm{H})$.

${ }^{13} \mathbf{C}$ NMR (126 MHz, $\left.\mathbf{C D C l}_{3}\right) \delta 171.4,138.8,138.1,129.1,128.5,127.8,127.6,124.3,120.0,73.0,70.4$, $37.8,29.7,29.2,26.1,25.7$.

HRMS (ESI) Calcd. for $\mathrm{C}_{20} \mathrm{H}_{26} \mathrm{NO}_{2}\left([\mathrm{M}+\mathrm{H}]^{+}\right): 312.1964$; Found: 312.1958 .

IR (neat) 3305, 3037, 2935, 2852, 1657, 1596, 1530, 1497, 1442, 1362, 1296, 1099, 737, 695, $499 \mathrm{~cm}^{-1}$. 
<smiles>N#Cc1ccc(OCCCCCCC(=O)Nc2ccccc2)cc1</smiles>

7-(4-cyanophenoxy)- $N$-phenylheptanamide (3g) Prepared according to general procedure using $1 \mathbf{a}(10 \mu \mathrm{L}$, $0.1 \mathrm{mmol})$ and $2 \mathrm{~g}(0.065 \mathrm{~g}, 0.2 \mathrm{mmol})$. The crude materials were combined from two parallel experiments and were purified by silica gel chromatography (hexane/ethyl acetate $=80: 20, \mathrm{Rf}=0.07$ ) to afford the title compound as a white solid $(0.030 \mathrm{~g}, 47 \%)$.

${ }^{1}$ H NMR (500 MHz, CDCl $) \delta 7.56(\mathrm{~d}, J=8.9 \mathrm{~Hz}, 2 \mathrm{H}), 7.51(\mathrm{~d}, J=7.7 \mathrm{~Hz}, 2 \mathrm{H}), 7.32(\mathrm{t}, J=7.9 \mathrm{~Hz}, 2 \mathrm{H})$, $7.15(\mathrm{bs}, 1 \mathrm{H}), 7.10(\mathrm{t}, J=7.3 \mathrm{~Hz}, 1 \mathrm{H}), 6.92(\mathrm{~d}, J=8.8 \mathrm{~Hz}, 2 \mathrm{H}), 3.99(\mathrm{t}, J=6.4 \mathrm{~Hz}, 2 \mathrm{H}), 2.38(\mathrm{t}, J=7.4 \mathrm{~Hz}, 2 \mathrm{H}), 1.80$ (dquintet, $J=15.0,5.4 \mathrm{~Hz}, 4 \mathrm{H}), 1.53-1.44(\mathrm{~m}, 4 \mathrm{H})$.

${ }^{13}$ C NMR (126 MHz, CDCl 3$) \delta 171.2,169.1,162.5,138.0,134.1,129.2,124.4,119.8,119.5,115.3,68.3$, 37.7, 29.0, 28.9, 25.9, 25.5.

HRMS (ESI) Calcd. for $\mathrm{C}_{20} \mathrm{H}_{23} \mathrm{~N}_{2} \mathrm{O}_{2}\left([\mathrm{M}+\mathrm{H}]^{+}\right)$: 323.1760; Found: 323.1762 .

IR (neat) 3310, 2939, 2850, 2216, 1663, 1597, 1526, 1506, 1438, 1301, 1259, 1170, 838, $501 \mathrm{~cm}^{-1}$.<smiles>O=C(CCCCCCOc1ccc(C(F)(F)F)cc1)Nc1ccccc1</smiles>

$\mathrm{N}$-phenyl-7-(4-(trifluoromethyl)phenoxy)heptanamide (3h) Prepared according to general procedure using $1 \mathrm{a}(10 \mu \mathrm{L}, 0.1 \mathrm{mmol})$ and $\mathbf{2 h}(0.074 \mathrm{~g}, 0.2 \mathrm{mmol})$. The crude materials were combined from two parallel experiments and were purified by silica gel chromatography (hexane/ethyl acetate $=80: 20, R f=0.18$ ) to afford the title compound as a white solid $(0.037 \mathrm{~g}, 51 \%)$.

${ }^{1}$ H NMR (500 MHz, CDCl$) \delta 7.52(\mathrm{t}, J=7.7 \mathrm{~Hz}, 4 \mathrm{H}), 7.32(\mathrm{t}, J=7.9 \mathrm{~Hz}, 2 \mathrm{H}), 7.16(\mathrm{~s}, 1 \mathrm{H}), 7.10(\mathrm{t}, J=7.4$ $\mathrm{Hz}, 1 \mathrm{H}), 6.93$ (d, $J=8.6 \mathrm{~Hz}, 2 \mathrm{H}), 3.99$ (t, $J=6.4 \mathrm{~Hz}, 2 \mathrm{H}), 2.37$ (t, $J=7.4 \mathrm{~Hz}, 2 \mathrm{H}), 1.79$ (td, $J=13.6,6.5 \mathrm{~Hz}, 4 \mathrm{H})$, $1.52-1.46(\mathrm{~m}, 4 \mathrm{H})$.

${ }^{13}$ C NMR (126 MHz, CDCl $) \delta 171.2,161.7,138.0,129.2,127.8,127.0,126.9,125.7,124.4,123.6,123.2$, $122.9,122.7,122.4,121.6,119.9,114.5,68.1,37.8,29.1,26.0,25.6$. Detail in CF coupling see SI page 28.

HRMS (ESI) Calcd. for $\mathrm{C}_{20} \mathrm{H}_{23} \mathrm{NO}_{2} \mathrm{~F}_{3}\left([\mathrm{M}+\mathrm{H}]^{+}\right): 366.1681$; Found: 366.1685 .

IR (neat) 3319, 2940, 2880, 1658, 1518, 1441, 1225, 1177, 694, $637 \mathrm{~cm}^{-1}$.<smiles>COC(=O)c1ccc(OCCCCCCC(=O)Nc2ccccc2)cc1</smiles>

methyl 4-((7-oxo-7-(phenylamino)heptyl)oxy)benzoate (3i) Prepared according to general procedure using $1 \mathbf{a}(10 \mu \mathrm{L}, 0.1 \mathrm{mmol})$ and $2 \mathbf{i}(0.072 \mathrm{~g}, 0.2 \mathrm{mmol})$. The crude materials were combined from two parallel experiments and were purified by silica gel chromatography (hexane/ethyl acetate $=80: 20, \mathrm{Rf}=0.11$ ) to afford the title compound as a white solid $(0.037 \mathrm{~g}, 51 \%)$.

${ }^{1}$ H NMR (500 MHz, CDCl $) \delta$ 7.98-7.96 (d, $\left.J=8.9 \mathrm{~Hz}, 2 \mathrm{H}\right), 7.51(\mathrm{~d}, J=7.8 \mathrm{~Hz}, 2 \mathrm{H}), 7.31(\mathrm{t}, J=7.8 \mathrm{~Hz}$, 2H), 7.14-7.10 (m, 2H), $6.89(\mathrm{~d}, J=8.9 \mathrm{~Hz}, 2 \mathrm{H}), 4.00(\mathrm{t}, J=6.4 \mathrm{~Hz}, 2 \mathrm{H}), 3.88(\mathrm{~s}, 3 \mathrm{H}), 2.37(\mathrm{t}, J=7.4 \mathrm{~Hz}, 2 \mathrm{H}), 1.79$ (dquintet, $J=20.8,7.0 \mathrm{~Hz}, 4 \mathrm{H}), 1.49(\mathrm{dq}, J=24.5,7.7 \mathrm{~Hz}, 4 \mathrm{H})$.

${ }^{13} \mathbf{C}$ NMR (126 MHz, $\left.\mathbf{C D C l}_{3}\right) \delta 171.2,167.1,163.0,138.0,131.7,129.2,124.4,122.5,119.8,114.2,68.1$, $52.0,37.8,29.1,26.0,25.6$.

HRMS (ESI) Calcd. for $\mathrm{C}_{21} \mathrm{H}_{26} \mathrm{NO}_{4}\left([\mathrm{M}+\mathrm{H}]^{+}\right)$: 356.1862; Found: 356.1858 .

IR (neat) 3325, 2944, 2864, 1711, 1668, 1605, 1527, 1439, 1259, 1170, 1107, 693, $501 \mathrm{~cm}^{-1}$. 


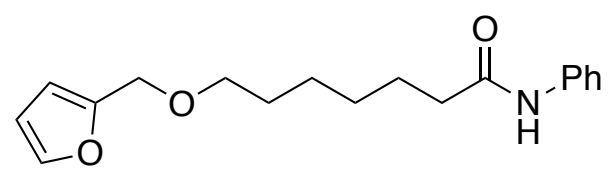

7-(furan-2-ylmethoxy)- $N$-phenylheptanamide (3j)Prepared according to general procedure using $\mathbf{1 a}$ (10 $\mu \mathrm{L}, 0.1 \mathrm{mmol})$ and $2 \mathbf{j}(0.052 \mathrm{~g}, 0.2 \mathrm{mmol})$. The crude materials were combined from two parallel experiments and were purified by silica gel chromatography (hexane/ethyl acetate $=80: 20, \mathrm{Rf}=0.10$ ) to afford the title compound as a light yellow oil $(0.033 \mathrm{~g}, 55 \%)$.

${ }^{1} \mathbf{H}$ NMR (500 MHz, CDCl $) \delta 7.51(\mathrm{~d}, J=7.8 \mathrm{~Hz}, 2 \mathrm{H}), 7.39-7.39(\mathrm{~m}, 2 \mathrm{H}), 7.32(\mathrm{t}, J=7.9 \mathrm{~Hz}, 2 \mathrm{H}), 7.10$ $(\mathrm{m}, 2 \mathrm{H}), 6.41(\mathrm{~m}, 1 \mathrm{H}), 4.36(\mathrm{~s}, 2 \mathrm{H}), 3.44(\mathrm{t}, J=6.5 \mathrm{~Hz}, 2 \mathrm{H}), 2.35(\mathrm{t}, J=7.5 \mathrm{~Hz}, 2 \mathrm{H}), 1.73$ (quintet, $J=7.2 \mathrm{~Hz}, 2 \mathrm{H})$, 1.60 (quintet, $J=6.6 \mathrm{~Hz}, 2 \mathrm{H}), 1.40(\mathrm{~m}, 4 \mathrm{H})$.

${ }^{13} \mathbf{C}$ NMR (126 MHz, $\left.\mathbf{C D C l}_{3}\right) \delta 171.3,143.4,140.7,138.1,129.2,124.3,122.7,119.9,110.5,70.2,64.2$, $37.9,29.7,29.2,26.1,25.7$.

HRMS (ESI) Calcd. for $\mathrm{C}_{18} \mathrm{H}_{24} \mathrm{NO}_{3}\left([\mathrm{M}+\mathrm{H}]^{+}\right)$: 302.1756; Found: 302.1751 .

IR (neat) 3304, 2928, 2856, 1664, 1597, 1535, 1442, 1094, 693, $498 \mathrm{~cm}^{-1}$.<smiles>O=C(CCCCCCn1ccc2ccccc21)Nc1ccccc1</smiles>

7-(1H-indol-1-yl)- $N$-phenylheptanamide (3k) Prepared according to general procedure using $1 \mathrm{a}(10 \mu \mathrm{L}$, $0.1 \mathrm{mmol})$ and $2 \mathbf{k}(0.065 \mathrm{~g}, 0.2 \mathrm{mmol})$. The crude materials were combined from two parallel experiments and were purified by silica gel chromatography (hexane/ethyl acetate $=80: 20, \mathrm{Rf}=0.16$ ) to afford the title compound as a white solid $(0.030 \mathrm{~g}, 47 \%)$.

${ }^{1} \mathbf{H}$ NMR $\left(500 \mathrm{MHz}, \mathbf{C D C l}_{3}\right) \delta 7.64(\mathrm{dd}, J=7.9,0.9 \mathrm{~Hz}, 1 \mathrm{H}), 7.49(\mathrm{~d}, J=7.9 \mathrm{~Hz}, 2 \mathrm{H}), 7.32(\mathrm{dt}, J=13.5$, $7.2 \mathrm{~Hz}, 3 \mathrm{H}), 7.21(\mathrm{ddd}, J=8.1,7.1,1.0 \mathrm{~Hz}, 1 \mathrm{H}), 7.14(\mathrm{~d}, J=8.8 \mathrm{~Hz}, 1 \mathrm{H}), 7.12-7.09(\mathrm{~m}, 3 \mathrm{H}), 6.49$ (dd, $J=3.1,0.8$ $\mathrm{Hz}, 1 \mathrm{H}$ ), 4.12 (t, $J=7.0 \mathrm{~Hz}, 2 \mathrm{H}$ ), $2.27(\mathrm{t}, J=7.5 \mathrm{~Hz}, 2 \mathrm{H}), 1.85$ (quintet, $J=7.2 \mathrm{~Hz}, 2 \mathrm{H}$ ), 1.69 (quintet, $J=7.4 \mathrm{~Hz}$, $2 \mathrm{H}), 1.39-1.33(\mathrm{~m}, 4 \mathrm{H})$.

${ }^{13}$ C NMR (126 MHz, CDCl $) \delta 171.2,138.0,136.1,129.1,128.7,128.0,124.3,121.5,121.1,119.9,119.3$, 109.5, 101.0, 46.4, 37.6, 30.1, 28.9, 26.8, 25.4.

HRMS (ESI) Calcd. for $\mathrm{C}_{21} \mathrm{H}_{25} \mathrm{~N}_{2} \mathrm{O}\left([\mathrm{M}+\mathrm{H}]^{+}\right)$: 321.1964; Found: 321.1967.

IR (neat) 3254, 2930, 2855, 1663, 1595, 1439, 1310, 1131, 747, $502 \mathrm{~cm}^{-1}$.<smiles>O=C(Nc1ccccc1)C1CCCCC1</smiles>

$N$-phenylcyclohexanecarboxamide (3l) Prepared according to general procedure using 1a $(10 \mu \mathrm{L}, 0.1 \mathrm{mmol})$ and $2 \mathrm{I}(129 \mu \mathrm{L}, 0.2 \mathrm{mmol})$. The crude materials were combined from two parallel experiments and were purified by silica gel chromatography (hexane/ethyl acetate $=80: 20, \mathrm{Rf}=0.26$ ) to afford the title compound as a white solid $(0.032 \mathrm{~g}, 80 \%)$.

${ }^{1}$ H NMR (500 MHz, CDCl $) \delta 7.53(\mathrm{~d}, J=7.8 \mathrm{~Hz}, 2 \mathrm{H}), 7.31(\mathrm{t}, J=8.0 \mathrm{~Hz}, 2 \mathrm{H}), 7.21(\mathrm{~s}, 1 \mathrm{H}), 7.09$ (t, $J=$ $7.4 \mathrm{~Hz}, 1 \mathrm{H}), 2.23(\mathrm{tt}, J=11.7,3.5 \mathrm{~Hz}, 1 \mathrm{H}), 1.97-1.94(\mathrm{~m}, 2 \mathrm{H}), 1.86-1.82(\mathrm{~m}, 2 \mathrm{H}), 1.70$ (ddt, $J=9.0,3.2,1.6 \mathrm{~Hz}, 1 \mathrm{H})$, $1.59-1.51(\mathrm{~m}, 2 \mathrm{H}), 1.35-1.23(\mathrm{~m}, 3 \mathrm{H})$.

${ }^{13}$ C NMR (126 MHz, CDCl $) \delta 174.5,138.2,129.1,124.2,119.9,46.7,29.8,25.8$.

HRMS (ESI) Calcd. for $\mathrm{C}_{13} \mathrm{H}_{18} \mathrm{NO}\left([\mathrm{M}+\mathrm{H}]^{+}\right)$: 204.1388; Found: 204.1389.

IR (neat) 3310, 2922, 2850, 1660, 1598, 1528, 1500, 1497, 1296, 1251, 1177, 1133, 731, 689, 585, 507, 439

$\mathrm{cm}^{-1}$. 


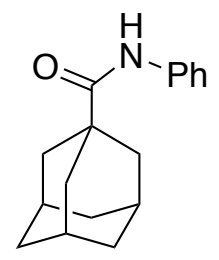

(3r,5r,7r)-N-phenyladamantane-1-carboxamide (3m) Prepared according to general procedure using $1 \mathrm{a}$ $(10 \mu \mathrm{L}, 0.1 \mathrm{mmol})$ and $\mathbf{2 m}(0.26 \mathrm{~g}, 0.2 \mathrm{mmol})$. The crude materials were combined from two parallel experiments and were purified by silica gel chromatography (hexane/ethyl acetate $=80: 20, \mathrm{Rf}=0.24$ ) to afford the title compound as a white solid $(0.044 \mathrm{~g}, 86 \%)$.

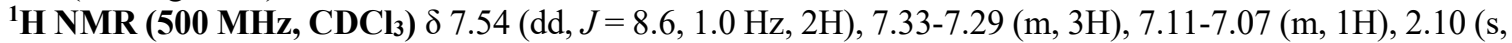
$3 \mathrm{H}), 1.97(\mathrm{~d}, J=2.6 \mathrm{~Hz}, 6 \mathrm{H}), 1.76(\mathrm{q}, J=11.1 \mathrm{~Hz}, 6 \mathrm{H})$.

${ }^{13}$ C NMR (126 MHz, $\left.\mathbf{C D C l}_{3}\right) \delta 176.2,138.2,129.1,124.2,120.0,41.6,39.4,36.6,28.3$.

HRMS (ESI) Calcd. for $\mathrm{C}_{17} \mathrm{H}_{22} \mathrm{NO}\left([\mathrm{M}+\mathrm{H}]^{+}\right): 256.1701$; Found: 256.1703 .

IR (neat) 3274, 2896, 2849, 1643, 1596, 1536, 1489, 1435, 1327, 1307, 1252, 754, 694, $512 \mathrm{~cm}^{-1}$.<smiles>CC1(C)O[C@H]2O[C@H](CC(=O)Nc3ccccc3)[C@H]3OC(C)(C)O[C@H]3[C@H]2O1</smiles>

$N$-phenyl-2-((3aR,5R,5aS,8a $S, 8 \mathrm{~b} R)-2,2,7,7$-tetramethyltetrahydro-5H-bis([1,3]dioxolo) [4,5- $b: 4 ', 5 '-$

d]pyran-5-yl)acetamide (3n) Prepared according to general procedure using $1 \mathbf{a}(10 \mu \mathrm{L}, 0.1 \mathrm{mmol})$ and $2 \mathrm{n}(0.074 \mathrm{~g}$, $0.2 \mathrm{mmol})$. The crude materials were combined from two parallel experiments and were purified by silica gel chromatography (hexane/ethyl acetate $=80: 20, \mathrm{Rf}=0.19)$ to afford the title compound as a white solid $(0.037 \mathrm{~g}, 51 \%)$.

${ }^{1} \mathbf{H}$ NMR $\left(500 \mathbf{M H z} \mathbf{C D C l}_{3}\right) \delta 8.32(\mathrm{~s}, 1 \mathrm{H}), 7.56(\mathrm{~d}, J=7.6 \mathrm{~Hz}, 2 \mathrm{H}), 7.30(\mathrm{t}, J=7.9 \mathrm{~Hz}, 2 \mathrm{H}), 7.08(\mathrm{t}, J=$ $7.4 \mathrm{~Hz}, 1 \mathrm{H}), 5.62(\mathrm{~d}, J=4.9 \mathrm{~Hz}, 1 \mathrm{H}), 4.65(\mathrm{dd}, J=7.9,2.5 \mathrm{~Hz}, 1 \mathrm{H}), 4.38(\mathrm{t}, J=2.5 \mathrm{~Hz}, 1 \mathrm{H}), 4.26(\mathrm{~d}, J=9.8 \mathrm{~Hz}, 1 \mathrm{H})$, $4.19(\mathrm{dd}, J=7.9,1.7 \mathrm{~Hz}, 1 \mathrm{H}), 2.84(\mathrm{dd}, J=16.4,9.9 \mathrm{~Hz}, 1 \mathrm{H}), 2.54(\mathrm{dd}, J=16.4,2.8 \mathrm{~Hz}, 1 \mathrm{H}), 1.52(\mathrm{~s}, 3 \mathrm{H}), 1.47$ (s, $3 \mathrm{H}), 1.36(\mathrm{~d}, J=3.8 \mathrm{~Hz}, 6 \mathrm{H})$.

${ }^{13}$ C NMR (126 MHz, $\left.\mathbf{C D C l}_{3}\right) \delta 169.1,138.4,129.0,124.1,119.9,109.8,109.7,109.5,96.7,72.8,70.8,70.6$, $65.1,38.7,26.1,25.1,24.5$.<smiles>CCCCCCCCC(=O)Nc1ccccc1</smiles>

$N$-phenylnonanamide (3o) Prepared according to general procedure using $1 \mathbf{a}(10 \mu \mathrm{L}, 0.1 \mathrm{mmol})$ and 20 (36 $\mu \mathrm{L}, 0.2 \mathrm{mmol})$. The crude materials were combined from two parallel experiments and were purified by silica gel chromatography (hexane/ethyl acetate $=90: 10, \mathrm{Rf}=0.19)$ to afford the title compound as a white solid $(0.038 \mathrm{~g}$, $82 \%)^{6}$

${ }^{1} \mathbf{H}$ NMR $(500$ MHz, CDCl $) \delta 7.51(\mathrm{~d}, J=8.0 \mathrm{~Hz}, 2 \mathrm{H}), 7.32(\mathrm{t}, J=7.8 \mathrm{~Hz}, 2 \mathrm{H}), 7.11-7.08(\mathrm{~m}, 2 \mathrm{H}), 2.35(\mathrm{t}$, $J=7.5 \mathrm{~Hz}, 2 \mathrm{H}), 1.76-1.70(\mathrm{~m}, 2 \mathrm{H}), 1.38-1.27(\mathrm{~m}, 10 \mathrm{H}), 0.88(\mathrm{t}, J=6.5 \mathrm{~Hz}, 3 \mathrm{H})$.

${ }^{13}$ C NMR (126 MHz, $\left.\mathbf{C D C l}_{3}\right) \delta 129.2,124.3,119.9,38.1,32.0,29.5,29.4,29.3,25.8,22.8,14.2$.

HRMS (ESI) Calcd. for $\mathrm{C}_{15} \mathrm{H}_{24} \mathrm{NO}\left([\mathrm{M}+\mathrm{H}]^{+}\right)$: 234.1858; Found: 234.1864 .

IR (neat) 3303, 2916, 2849, 1655, 1599, 1536, 1443, 754, 600, $503 \mathrm{~cm}^{-1}$.<smiles>CCC(C)c1ccc(NC(=O)CCCc2ccccc2)cc1</smiles> 
N-(4-(tert-butyl)phenyl)-4-phenylbutanamide (3p) Prepared according to general procedure using 1p $(0.018 \mathrm{~g}, 0.1 \mathrm{mmol})$ and $2 \mathrm{a}(32 \mu \mathrm{L}, 0.2 \mathrm{mmol})$. The crude materials were combined from two parallel experiments and were purified by silica gel chromatography (hexane/ethyl acetate $=90: 10, \mathrm{Rf}=0.09$ ) to afford the title compound as a brown solid $(0.054 \mathrm{~g}, 92 \%)$.

${ }^{1}$ H NMR (500 MHz, $\left.\mathbf{C D C l}_{3}\right) \delta 7.32(\mathrm{~d}, J=8.6 \mathrm{~Hz}, 2 \mathrm{H}), 7.24-7.18(\mathrm{~m}, 4 \mathrm{H}), 7.12-(\mathrm{m}, 3 \mathrm{H}), 7.01(\mathrm{~s}, 1 \mathrm{H}), 2.61$ (t, $J=7.4 \mathrm{~Hz}, 2 \mathrm{H}), 2.23$ (t, $J=7.4 \mathrm{~Hz}, 2 \mathrm{H}$ ), 1.97 (quintet, $J=7.4 \mathrm{~Hz}, 2 \mathrm{H}), 1.207 .09$ (s, 9H).

${ }^{13} \mathbf{C}$ NMR (126 MHz, CDCl $) \delta 170.9,147.3,141.5,135.4,128.7,128.6,126.2,125.9,119.8,36.9,35.2$, 31.5, 27.1.

HRMS (ESI) Calcd. for $\mathrm{C}_{20} \mathrm{H}_{26} \mathrm{NO}\left([\mathrm{M}+\mathrm{H}]^{+}\right)$: 296.2014; Found: 296.2013.

IR (neat) 3294, 2957, 1653, 1598, 1538, 1404, 1256, 834, 695, $472 \mathrm{~cm}^{-1}$.<smiles>O=C(CCCc1ccccc1)Nc1ccc(Br)cc1</smiles>

$N$-(4-bromophenyl)-4-phenylbutanamide (3q) Prepared according to general procedure using 1q $(0.02 \mathrm{~g}$, $0.1 \mathrm{mmol})$ and $2 \mathrm{a}(32 \mu \mathrm{L}, 0.2 \mathrm{mmol})$. The crude materials were combined from two parallel experiments and were purified by silica gel chromatography (hexane/ethyl acetate $=85: 15, \mathrm{Rf}=0.17$ ) to afford the title compound as a yellow solid $(0.049 \mathrm{~g}, 77 \%)$.

${ }^{1} \mathbf{H}$ NMR (500 MHz, $\left.\mathbf{C D C l}_{3}\right) \delta$ 7.43-7.39 (m, 4H), 7.32-7.27 (m, 2H), 7.21 (dd, $\left.J=13.4,7.2 \mathrm{~Hz}, 4 \mathrm{H}\right), 2.71$ (t, $J=7.4 \mathrm{~Hz}, 2 \mathrm{H}), 2.33(\mathrm{t}, J=7.5 \mathrm{~Hz}, 2 \mathrm{H}), 2.06$ (quintet, $J=7.4 \mathrm{~Hz}, 2 \mathrm{H}$ ).

${ }^{13} \mathbf{C}$ NMR (126 MHz, $\left.\mathbf{C D C l}_{3}\right) \delta 171.1,141.3,132.1,128.6,128.6,128.6,126.2,121.4,116.9,77.2,36.8$, $35.2,26.8$.

HRMS (ESI) Calcd. for $\mathrm{C}_{16} \mathrm{H}_{17} \mathrm{NOBr}\left([\mathrm{M}+\mathrm{H}]^{+}\right)$: 318.0494; Found: 318.0497.

IR (neat) 3289, 3060, 2866, 1657, 1587, 1516, 1485, 1392, 1283, 1130, 1007, 813, 740, 695, 695, $497 \mathrm{~cm}^{-}$ 1.<smiles>O=C(CCCc1ccccc1)Nc1ccc(I)cc1</smiles>

N-(4-iodophenyl)-4-phenylbutanamide (3r) Prepared according to general procedure using $1 \mathbf{r}(0.025 \mathrm{~g}$, $0.1 \mathrm{mmol})$ and $2 \mathrm{a}(32 \mu \mathrm{L}, 0.2 \mathrm{mmol})$. The crude materials were combined from two parallel experiments and were purified by silica gel chromatography (hexane/ethyl acetate $=90: 10, \mathrm{Rf}=0.19$ ) to afford the title compound as a yellow solid $(0.047 \mathrm{~g}, 64 \%)$.

${ }^{1}$ H NMR (500 MHz, CDCl $) \delta 7.60(\mathrm{~d}, J=8.6 \mathrm{~Hz}, 2 \mathrm{H}), 7.31-7.26(\mathrm{~m}, 5 \mathrm{H}), 7.23-7.18(\mathrm{~m} \mathrm{3H}), 2.70(\mathrm{t}, J=$ $7.3 \mathrm{~Hz}, 2 \mathrm{H}), 2.33$ (t, $J=7.4 \mathrm{~Hz}, 2 \mathrm{H}), 2.06$ (q, $J=7.2 \mathrm{~Hz}, 2 \mathrm{H})$.

${ }^{13}$ C NMR (126 MHz, CDCl $) \delta 171.1,141.3,132.1,128.6,128.6,128.6,126.2,121.4,116.9,77.2,36.8$, $35.2,26.8$.

HRMS (ESI) Calcd. for $\mathrm{C}_{16} \mathrm{H}_{17} \mathrm{NOI}\left([\mathrm{M}+\mathrm{H}]^{+}\right)$: $366.0355,366.0344$; Found: 366.0353 .

IR (neat) 3292, 3024, 2942, 2352, 1660, 1581, 1513, 1389, 1280, 1129, 1003, 812, 748, 695, $489 \mathrm{~cm}^{-1}$.<smiles>O=C(CCCc1ccccc1)Nc1ccc(F)cc1</smiles>

$N$-(4-fluorophenyl)-4-phenylbutanamide (3s) Prepared according to general procedure using $1 \mathrm{~s}(21 \mu \mathrm{L}$, $0.1 \mathrm{mmol})$ and 2a $(32 \mu \mathrm{L}, 0.2 \mathrm{mmol})$. The crude materials were combined from two parallel experiments and were purified by silica gel chromatography (hexane/ethyl acetate $=85: 15, \mathrm{Rf}=0.16$ ) to afford the title compound as a colorless oil (0.028 g, 54\%). 
${ }^{1}$ H NMR (500 MHz, CDCl $)$ ) $\delta 7.46-7.43(\mathrm{~m}, 2 \mathrm{H}), 7.30(\mathrm{t}, J=7.4 \mathrm{~Hz}, 2 \mathrm{H}), 7.21(\mathrm{t}, J=7.6 \mathrm{~Hz}, 2 \mathrm{H}), 7.10$ (bs, $1 \mathrm{H}), 7.00(\mathrm{t}, J=8.6 \mathrm{~Hz}, 2 \mathrm{H}), 6.95-6.91(\mathrm{~m}, 1 \mathrm{H}), 2.71(\mathrm{t}, J=7.4 \mathrm{~Hz}, 2 \mathrm{H}), 2.33(\mathrm{t}, J=7.4 \mathrm{~Hz}, 2 \mathrm{H}), 2.10-2.04(\mathrm{~m}, 2 \mathrm{H})$.

${ }^{13}$ C NMR (126 MHz, CDCl $) \delta 171.0,160.4,158.5,141.4,134.0,128.7,128.6,126.2,121.8,121.7,115.8$, 115.7, 36.7, 35.2, 26.9. Detailed CF splitting information can be found in SI page 40.

HRMS (ESI) Calcd. for $\mathrm{C}_{16} \mathrm{H}_{17} \mathrm{NOF}\left([\mathrm{M}+\mathrm{H}]^{+}\right)$: 258.1294; Found: 258.1292.

IR (neat) 3287, 3063, 3027, 1658, 1508, 1407, 1152, 831, 740, 696, $492 \mathrm{~cm}^{-1}$.<smiles>O=C(CCCc1ccccc1)Nc1cccc(Cl)c1</smiles>

N-(3-chlorophenyl)-4-phenylbutanamide (3t) Prepared according to general procedure using $1 \mathrm{t}$ ( $0.16 \mathrm{~g}$, $0.1 \mathrm{mmol})$ and $2 \mathrm{a}(32 \mu \mathrm{L}, 0.2 \mathrm{mmol})$. The crude materials were combined from two parallel experiments and were purified by silica gel chromatography (hexane/ethyl acetate $=90: 10, \mathrm{Rf}=0.08$ ) to afford the title compound as a yellow oil $(0.031 \mathrm{~g}, 57 \%)$ with around $10 \%$ inseparable impurities.

${ }^{1}$ H NMR (500 MHz, CDCl$) \delta 7.63(\mathrm{~s}, 1 \mathrm{H}), 7.33-7.28(\mathrm{~m}, 3 \mathrm{H}), 7.33-7.27(\mathrm{~m}, J=9.5,6.2 \mathrm{~Hz}, 4 \mathrm{H}), 7.17-$ $7.13(\mathrm{~m}, 2 \mathrm{H}), 2.71(\mathrm{t}, J=7.4 \mathrm{~Hz}, 2 \mathrm{H}), 2.34(\mathrm{t}, J=7.4 \mathrm{~Hz}, 2 \mathrm{H}), 2.07$ (quintet, $J=7.5 \mathrm{~Hz}, 2 \mathrm{H}$ ).

${ }^{13}$ C NMR (126 MHz, $\left.\mathbf{C D C l}_{3}\right) \delta 171.1,141.3,130.1,128.7,128.6,128.6,126.3,124.4,120.0,117.8,35.1$, 33.2, 26.8.

HRMS (ESI) Calcd. for $\mathrm{C}_{16} \mathrm{H}_{17} \mathrm{NOCl}\left([\mathrm{M}+\mathrm{H}]^{+}\right)$: 274.0999; Found: 274.1005.<smiles>COc1ccc(NC(=O)CCCc2ccccc2)cc1</smiles>

N-(4-methoxyphenyl)-4-phenylbutanamide (3u) Prepared according to general procedure using $1 \mathbf{u}(0.015$ $\mathrm{g}, 0.1 \mathrm{mmol})$ and $2 \mathrm{a}(32 \mu \mathrm{L}, 0.2 \mathrm{mmol})$. The crude materials were combined from two parallel experiments and were purified by silica gel chromatography (hexane/ethyl acetate $=80: 10, \mathrm{Rf}=0.20$ ) to afford the title compound as a brown solid (0.026 g, 47\%).

${ }^{1} \mathbf{H}$ NMR (500 MHz, CDCl$) \delta 7.39$ (d, $\left.J=8.9 \mathrm{~Hz}, 2 \mathrm{H}\right), 7.30(\mathrm{t}, J=7.5 \mathrm{~Hz}, 2 \mathrm{H}), 7.21$ (t, $\left.J=6.1 \mathrm{~Hz}, 3 \mathrm{H}\right)$, $7.10(\mathrm{~s}, 1 \mathrm{H}), 6.86-6.84(\mathrm{~m}, 2 \mathrm{H}), 3.78(\mathrm{~s}, 3 \mathrm{H}), 2.71(\mathrm{t}, J=7.4 \mathrm{~Hz}, 2 \mathrm{H}), 2.32(\mathrm{t}, J=7.5 \mathrm{~Hz}, 2 \mathrm{H}), 2.07$ (quintet, $J=7.4$ $\mathrm{Hz}, 2 \mathrm{H})$.

${ }^{13}$ C NMR (126 MHz, $\left.\mathbf{C D C l}_{3}\right) \delta 170.9,141.5,131.1,128.7,128.6,126.6,126.2,121.9,114.3,55.6,36.7$, $35.2,27.1$.

HRMS (ESI) Calcd. for $\mathrm{C}_{17} \mathrm{H}_{20} \mathrm{NO}_{2}\left([\mathrm{M}+\mathrm{H}]^{+}\right)$: 270.1494; Found: 270.1496 .

IR (neat) 3274, 3050, 2928, 1650, 1510, 1430, 1244, 1026, 824, 728, 695, $499 \mathrm{~cm}^{-1}$.<smiles>O=C(CCCc1ccccc1)Nc1ccc(OC(F)(F)F)cc1</smiles>

4-phenyl- $\mathrm{N}$-(4-(trifluoromethoxy)phenyl)butanamide (3v) Prepared according to general procedure using $1 \mathbf{v}(0.021 \mathrm{~g}, 0.1 \mathrm{mmol})$ and $2 \mathrm{a}(32 \mu \mathrm{L}, 0.2 \mathrm{mmol})$. The crude materials were combined from two parallel experiments and were purified by silica gel chromatography (hexane/ethyl acetate $=85: 15, \mathrm{Rf}=0.19$ ) to afford the title compound as a yellow solid $(0.048 \mathrm{~g}, 75 \%)$.

${ }^{1}$ H NMR (500 MHz, CDCl$) \delta 7.52(\mathrm{~d}, J=8.9 \mathrm{~Hz}, 2 \mathrm{H}), 7.30(\mathrm{t}, J=7.4 \mathrm{~Hz}, 2 \mathrm{H}), 7.21-7.15(\mathrm{~m}, 6 \mathrm{H}), 2.72(\mathrm{t}$, $J=7.4 \mathrm{~Hz}, 2 \mathrm{H}$ ), 2.35 (t, $J=7.5 \mathrm{~Hz}, 2 \mathrm{H}$ ), 2.07 (quintet, $J=7.4 \mathrm{~Hz}, 2 \mathrm{H}$ ).

${ }^{13}$ C NMR (126 MHz, $\left.\mathbf{C D C l}_{3}\right) \delta 171.4,145.6,141.6,136.9,128.9,126.5,123.0,122.1,121.9,121.2,119.8$, 37.0, 35.4, 27.1.(Unfortunately, no C-F coupling was observed)

HRMS (ESI) Calcd. for $\mathrm{C}_{17} \mathrm{H}_{17} \mathrm{NO}_{2} \mathrm{~F}_{3}\left([\mathrm{M}+\mathrm{H}]^{+}\right)$: 324.1211; Found: 324.1219 . 
<smiles>CSc1ccc(NC(=O)CCCc2ccccc2)cc1</smiles>

$\mathrm{N}$-(4-(methylthio)phenyl)-4-phenylbutanamide (3w) Prepared according to general procedure using $\mathbf{1 w}$ $(0.017 \mathrm{~g}, 0.1 \mathrm{mmol})$ and $2 \mathrm{a}(32 \mu \mathrm{L}, 0.2 \mathrm{mmol})$. The crude materials were combined from two parallel experiments and were purified by silica gel chromatography (hexane/ethyl acetate $=80: 10, \mathrm{Rf}=0.20$ ) to afford the title compound as a light pink solid $(0.042 \mathrm{~g}, 73 \%)$.

${ }^{1} \mathbf{H}$ NMR (500 MHz, $\left.\mathbf{C D C l}_{3}\right) \delta 7.43(\mathrm{~d}, J=8.6 \mathrm{~Hz}, 2 \mathrm{H}), 7.29(\mathrm{t}, J=7.5 \mathrm{~Hz}, 2 \mathrm{H}), 7.23-7-16(\mathrm{~m}, 5 \mathrm{H}), 7.16(\mathrm{~s}$, $1 \mathrm{H}), 2.71(\mathrm{~d}, J=7.4 \mathrm{~Hz}, 2 \mathrm{H}), 2.46(\mathrm{~s}, 2 \mathrm{H}), 2.33(\mathrm{t}, J=7.4 \mathrm{~Hz}, 2 \mathrm{H}), 2.06$ (quintet, $J=7.5 \mathrm{~Hz}, 2 \mathrm{H}$ ).

${ }^{13} \mathbf{C}$ NMR (126 MHz, $\left.\mathbf{C D C l}_{3}\right) \delta 171.0,141.4,135.7,133.6,128.7,128.6,128.2,126.2,120.6,36.8,35.2$, 26.9, 16.9.

HRMS (ESI) Calcd. for $\mathrm{C}_{17} \mathrm{H}_{20} \mathrm{NOS}\left([\mathrm{M}+\mathrm{H}]^{+}\right)$: 286.1266; Found: 286.1273 .

IR (neat) 3284, 3176, 2918, 1650, 1592, 1528, 1492, 1396, 1349, 1308, 1092, 821, 741, 696, $481 \mathrm{~cm}^{-1}$.<smiles>O=C(CCCc1ccccc1)Nc1ccc(O)cc1</smiles>

N-(4-hydroxyphenyl)-4-phenylbutanamide (3x) Prepared according to general procedure using pivalic acid protected $1 \mathbf{x}(0.017 \mathrm{~g}, 0.1 \mathrm{mmol})$ and $2 \mathrm{a}(32 \mu \mathrm{L}, 0.2 \mathrm{mmol})$. The crude materials were combined from two parallel experiments and were purified by silica gel chromatography (hexane/ethyl acetate $=70: 30, \mathrm{Rf}=0.08$ ) to afford the title compound as a reddish solid $(0.042 \mathrm{~g}, 51 \%)$.

${ }^{1} \mathbf{H}$ NMR $\left(500 \mathrm{MHz}, \mathbf{C D C l}_{3}\right) \delta 7.30(\mathrm{t}, J=7.8 \mathrm{~Hz}, 3 \mathrm{H}), 7.22-7.19(\mathrm{~m}, 3 \mathrm{H}), 6.96(\mathrm{~s}, 1 \mathrm{H}), 6.77(\mathrm{~d}, J=8.7 \mathrm{~Hz}$, $2 \mathrm{H}), 5.02(\mathrm{~s}, 1 \mathrm{H}), 2.72(\mathrm{t}, J=7.4 \mathrm{~Hz}, 2 \mathrm{H}), 2.33(\mathrm{t}, J=7.4 \mathrm{~Hz}, 2 \mathrm{H}), 2.10-2.04(\mathrm{~m}, 2 \mathrm{H})$.

${ }^{13}$ C NMR (126 MHz, $\left.\mathbf{C D C l}_{3}\right) \delta 171.0,141.5,131.0,128.7,128.6,126.2,115.8,36.7,35.2,27.1$.

HRMS (ESI) Calcd. for $\mathrm{C}_{16} \mathrm{H}_{18} \mathrm{NO}_{2}\left([\mathrm{M}+\mathrm{H}]^{+}\right): 256.1338$; Found: 256.1246 .<smiles>CC1(C)OB(c2ccc(NC(=O)CCCc3ccccc3)cc2)OC1(C)C</smiles>

4-phenyl- $N$-(4-(4,4,5,5-tetramethyl-1,3,2-dioxaborolan-2-yl)phenyl)butanamide $\quad(3 y) \quad$ Prepared according to general procedure using $1 \mathbf{y}(0.025 \mathrm{~g}, 0.1 \mathrm{mmol})$ and $2 \mathbf{a}(32 \mu \mathrm{L}, 0.2 \mathrm{mmol})$. The crude materials were combined from two parallel experiments and were purified by silica gel chromatography (hexane/ethyl acetate $=85$ : $15, \mathrm{Rf}=0.16)$ to afford the title compound as a brown solid $(0.051 \mathrm{~g}, 70 \%)$.

${ }^{1} \mathbf{H}$ NMR $\left(500 \mathrm{MHz}, \mathbf{C D C l}_{3}\right) \delta 7.76(\mathrm{~d}, J=8.1 \mathrm{~Hz}, 2 \mathrm{H}), 7.51(\mathrm{~d}, J=7.5 \mathrm{~Hz}, 2 \mathrm{H}), 7.31-7.28(\mathrm{~m}, 2 \mathrm{H}), 7.20-$ $7.17(\mathrm{~m}, 4 \mathrm{H}), 2.71(\mathrm{t}, J=7.4 \mathrm{~Hz}, 2 \mathrm{H}), 2.35(\mathrm{t}, J=7.4 \mathrm{~Hz}, 2 \mathrm{H}), 2.10-2.03(\mathrm{~m}, 2 \mathrm{H}), 1.34(\mathrm{~s}, 12 \mathrm{H})$.

${ }^{13}$ C NMR (126 MHz, CDCl $) \delta 171.3,141.5,140.7,136.0,134.2,128.7,128.6,126.2,118.6,83.9,37.0$, $35.2,26.9,25.0$.<smiles>O=C(CCCc1ccccc1)Nc1ccc2c(c1)OCO2</smiles>

$N$-(benzo $[d][1,3]$ dioxol-5-yl)-4-phenylbutanamide (3z) Prepared according to general procedure using pivalic acid protected $\mathbf{1 z}(0.017 \mathrm{~g}, 0.1 \mathrm{mmol})$ and $\mathbf{2 a}(32 \mu \mathrm{L}, 0.2 \mathrm{mmol})$. The crude materials were combined from two parallel experiments and were purified by silica gel chromatography (hexane/ethyl acetate $=80: 20, R f=0.17$ ) to afford the title compound as a reddish solid $(0.024 \mathrm{~g}, 52 \%)$. 


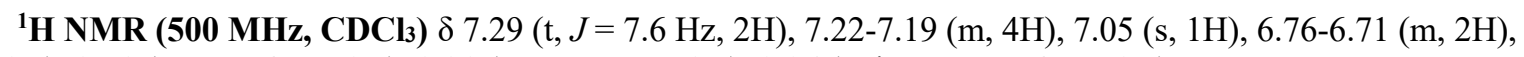
$5.93(\mathrm{~s}, 2 \mathrm{H}), 2.71(\mathrm{t}, J=7.4 \mathrm{~Hz}, 2 \mathrm{H}), 2.31(\mathrm{t}, J=7.5 \mathrm{~Hz}, 2 \mathrm{H}), 2.06$ (quintet, $J=7.4 \mathrm{~Hz}, 2 \mathrm{H})$.

${ }^{13}$ C NMR (126 MHz, CDCl $) \delta 170.9,141.5,132.2,128.7,128.6,128.5,126.2,113.1,108.2,103.0,101.4$, $36.8,35.2,27.0$.

HRMS (ESI) Calcd. for $\mathrm{C}_{17} \mathrm{H}_{18} \mathrm{NO}_{3}\left([\mathrm{M}+\mathrm{H}]^{+}\right)$: 284.1287 ; Found: 284.1292 .

IR (neat) 3273, 3060, 3025, 2919, 1650, 1502, 1487, 1447, 1209, 1130, 1097, 1035, 924, 695, $487 \mathrm{~cm}^{-1}$. 


\section{Mechanistic Studies}

\subsection{Stoichiometric Experiment}

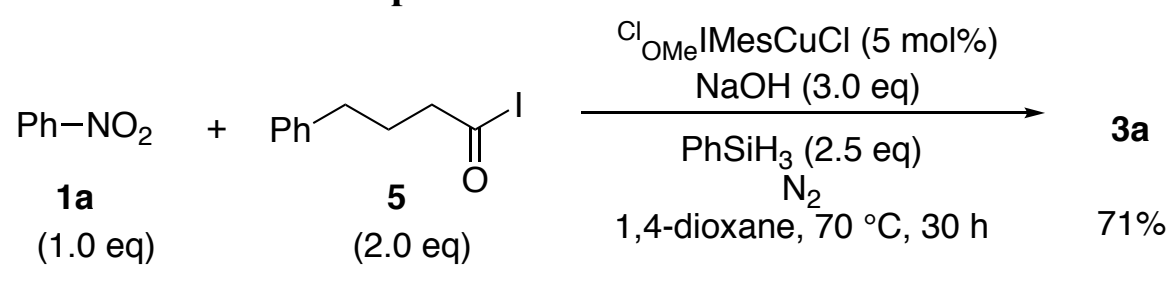

By Subjecting acyl iodide $5(0.055 \mathrm{~g}, 0.2 \mathrm{mmol}, 2.0 \mathrm{eq})$ to the optimized conditions under $\mathrm{N}_{2}$ atmosphere without $\mathrm{CO}, 71 \%$ amide compound was observed by ${ }^{1} \mathrm{H} \mathrm{NMR}$ in $\mathrm{CDCl}_{3}$.

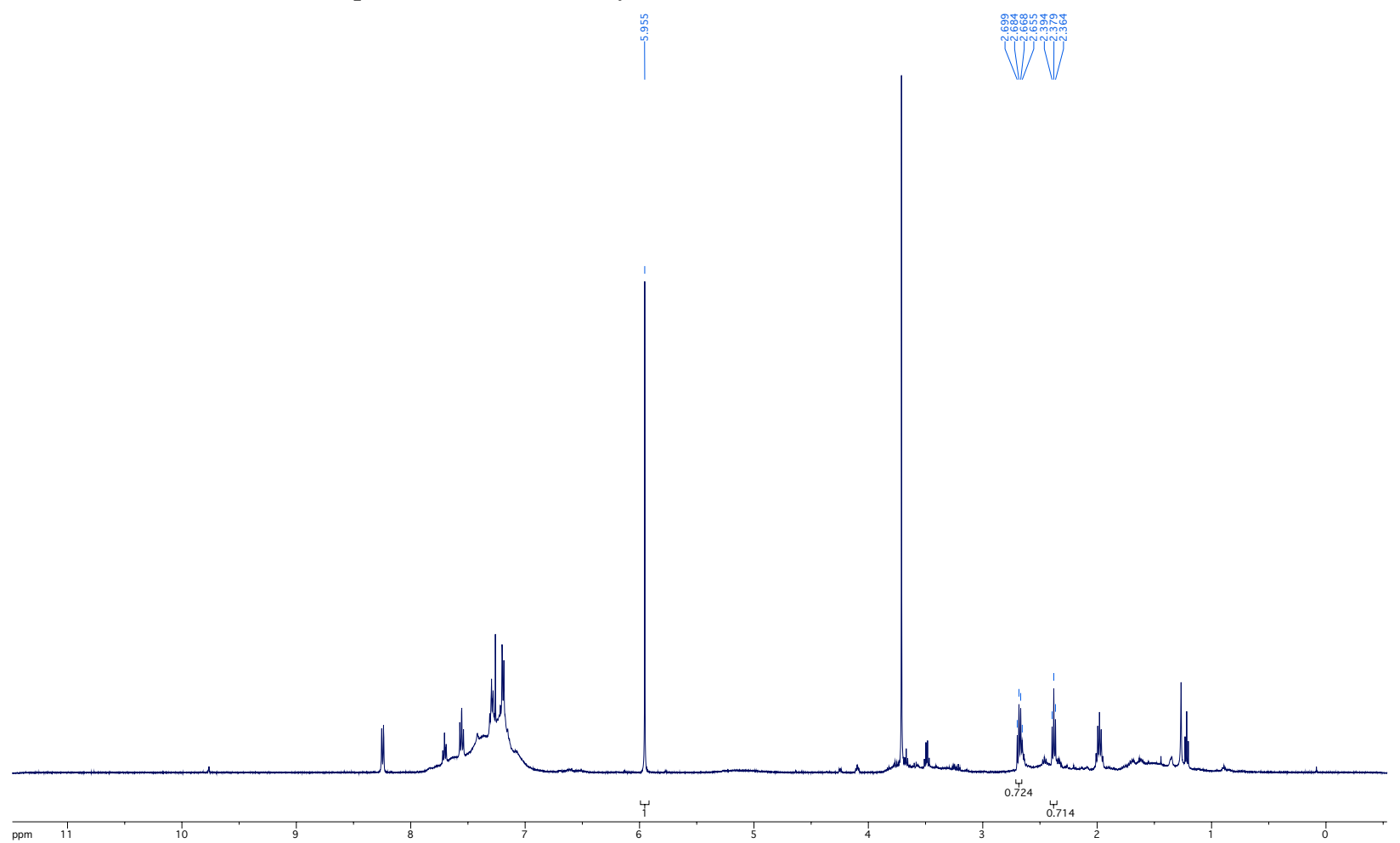

\subsection{Radical Clock Experiments}
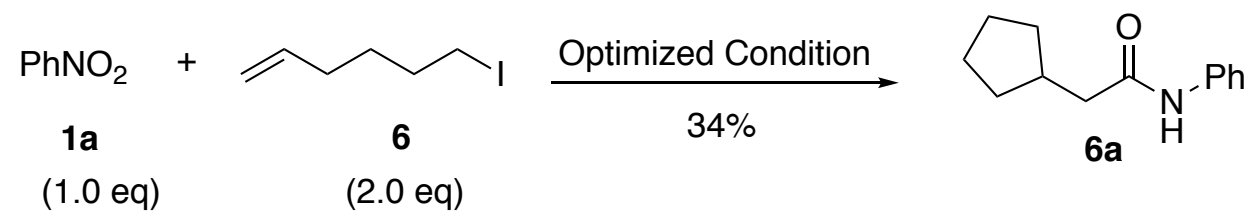

6-iodohex-1-ene (6) (0.042 g, $0.2 \mathrm{mmol}, 2.0 \mathrm{eq})$ was added to the reaction, and the ring-closed compound $\mathbf{6 a}$ was observed, and yield was determined by ${ }^{1} \mathrm{H}$ NMR in DMSO- $d_{6}$ according to reported literature. ${ }^{7}$ 


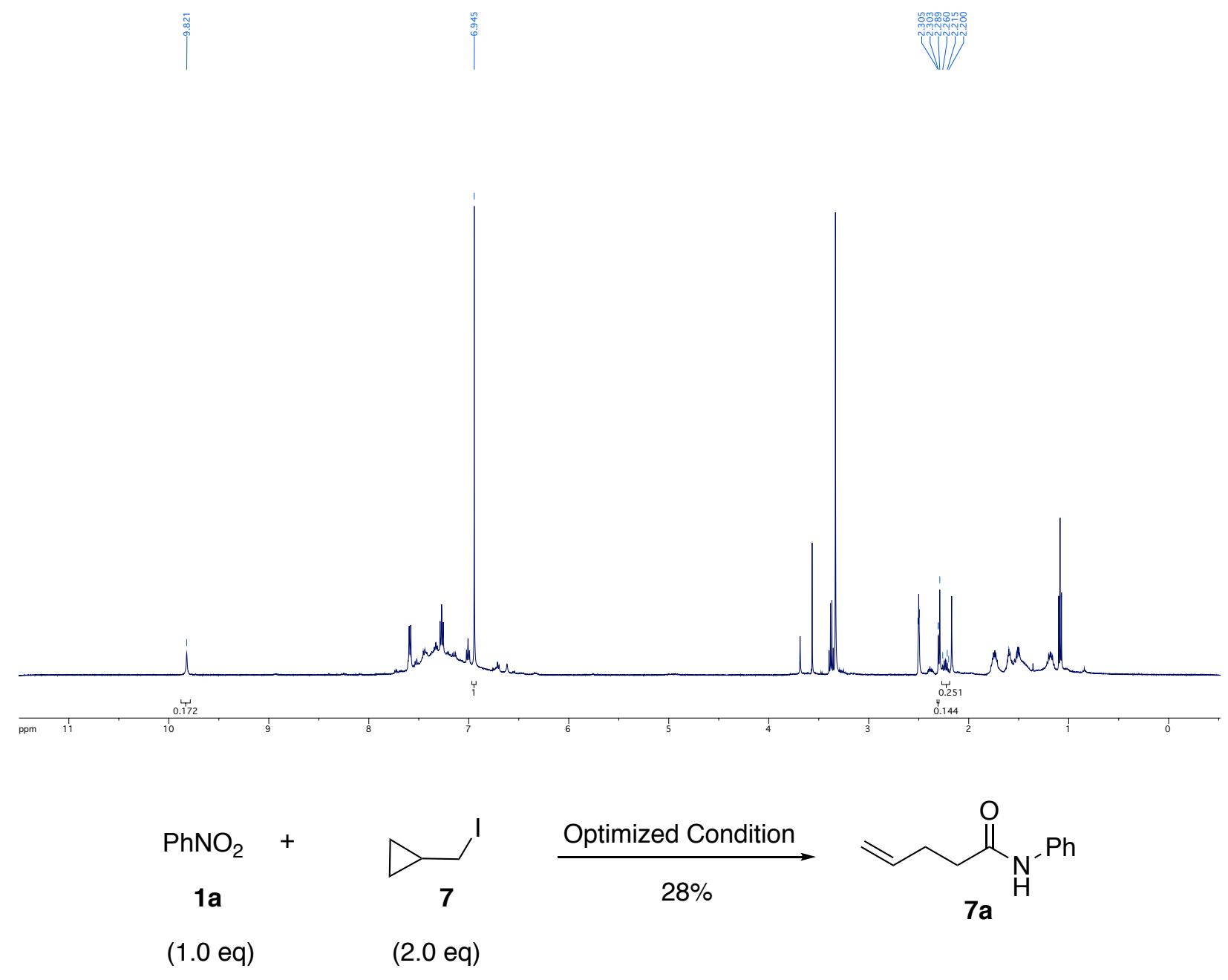

(iodomethyl)cyclopropane (7) ( $0.036 \mathrm{~g}, 0.2 \mathrm{mmol}, 2.0 \mathrm{eq})$ was added to the reaction, and the ring-opened compound 7a was observed, and yield was determined by ${ }^{1} \mathrm{H} \mathrm{NMR}$ in $\mathrm{CDCl}_{3}$ according to the reported literature. ${ }^{8}$

\subsection{TEMPO Experiment}

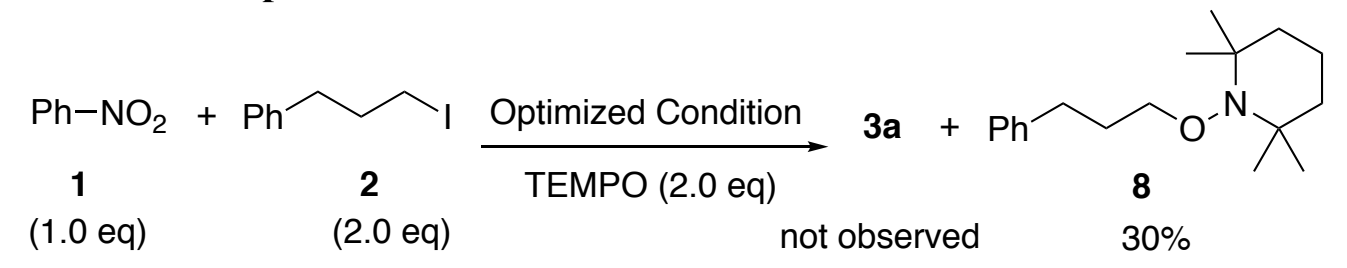

(2,2,6,6-tetramethylpiperidin-1-yl)oxidanyl ( $0.03 \mathrm{~g}, 0.2 \mathrm{mmol}, 2.0 \mathrm{eq})$ was added to the standard reaction, and no amide was observed. Instead TEMPO adduct compound $\mathbf{8}$ was observed and isolated. NMR spectrum was consistent with previous literature. ${ }^{4}$ 


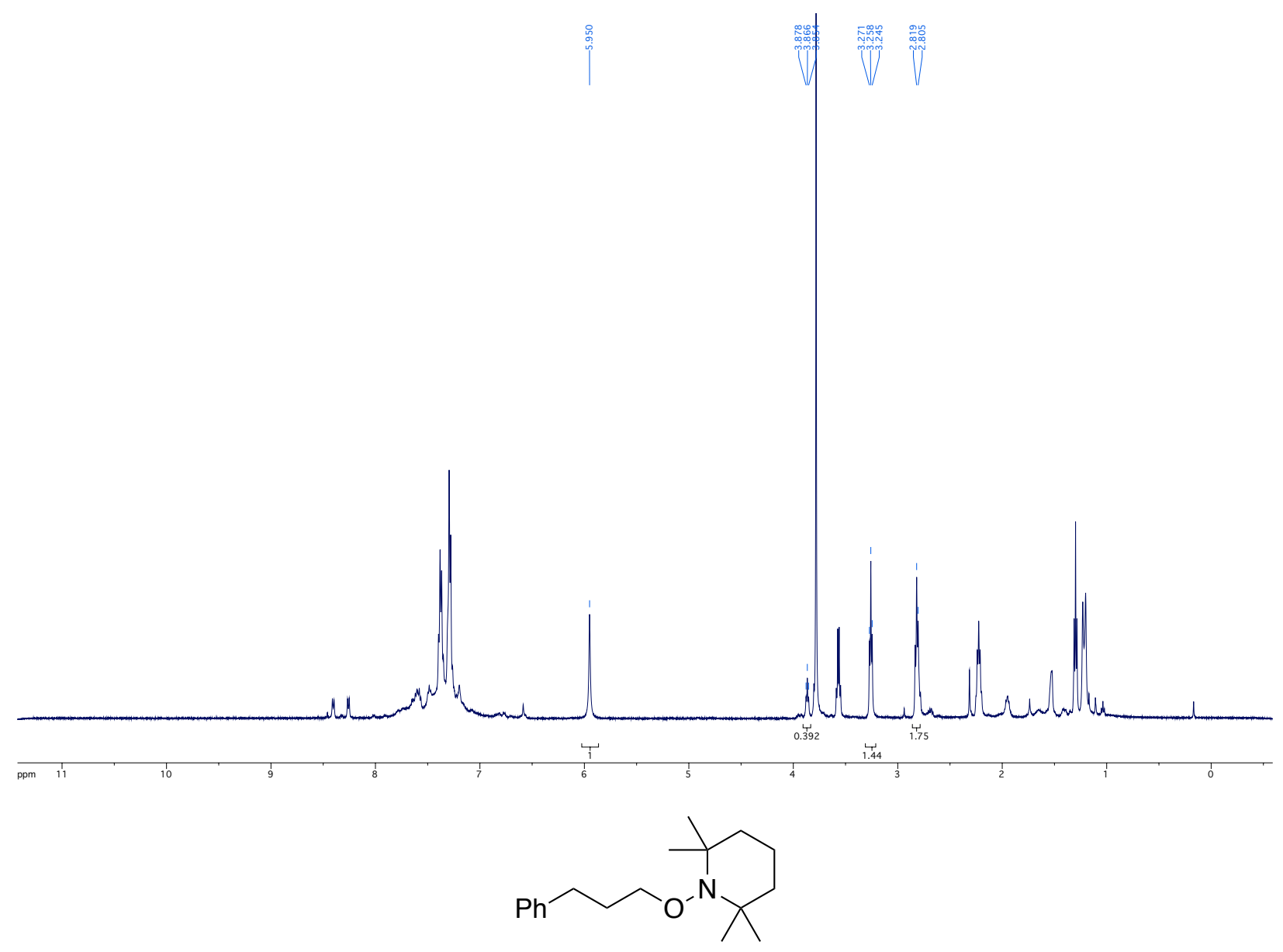

${ }^{1} \mathbf{H}$ NMR (500 MHz, $\left.\mathbf{C D C l}_{3}\right) \delta$ 7.29-7.26 (m, 2H), 7.22-7.20 (m, 2H), 7.19-7.16 (m, 1H), $3.77(\mathrm{t}, J=6.5 \mathrm{~Hz}$, 2H), $2.71(\mathrm{t}, J=8.0 \mathrm{~Hz}, 2 \mathrm{H}), 1.89-1.83(\mathrm{~m}, 2 \mathrm{H}), 1.55(\mathrm{~m}, 1 \mathrm{H}), 1.44(\mathrm{~d}, J=5.1 \mathrm{~Hz}, 4 \mathrm{H}), 1.32(\mathrm{~m}, 1 \mathrm{H}), 1.12(\mathrm{~d}, J=$ $14.1 \mathrm{~Hz}, 12 \mathrm{H})$.

${ }^{13}$ C NMR (126 MHz, $\left.\mathbf{C D C l}_{3}\right) \delta 128.51,128.41,125.8,59.8,39.8,33.2,32.9,30.7,20.3,17.3$

HRMS (ESI) Calcd. for $\mathrm{C}_{18} \mathrm{H}_{30} \mathrm{NO}\left([\mathrm{M}+\mathrm{H}]^{+}\right)$: 276.2327; Found: 276.2321 .

\subsection{Possible Reduction Intermediates from Nitroarene Reduction}

Beside the experiments shown in Scheme 2c, more control experiments with possible intermediates were carried out with 2.0 eq acyl iodide in the absence of hydrosilanes. Azoxybenzene provide none of the target compound. The rest of them performed the aminocarbonylation to some extent, although nitrosobenzene and azobenzene gave poor yields. Combining the results in scheme 2c, we cannot exclude other intermediates being relevant to amide formation by minor pathways. However, we still conclude aniline serve as the major intermediate in this reductive aminocarbonylation. 


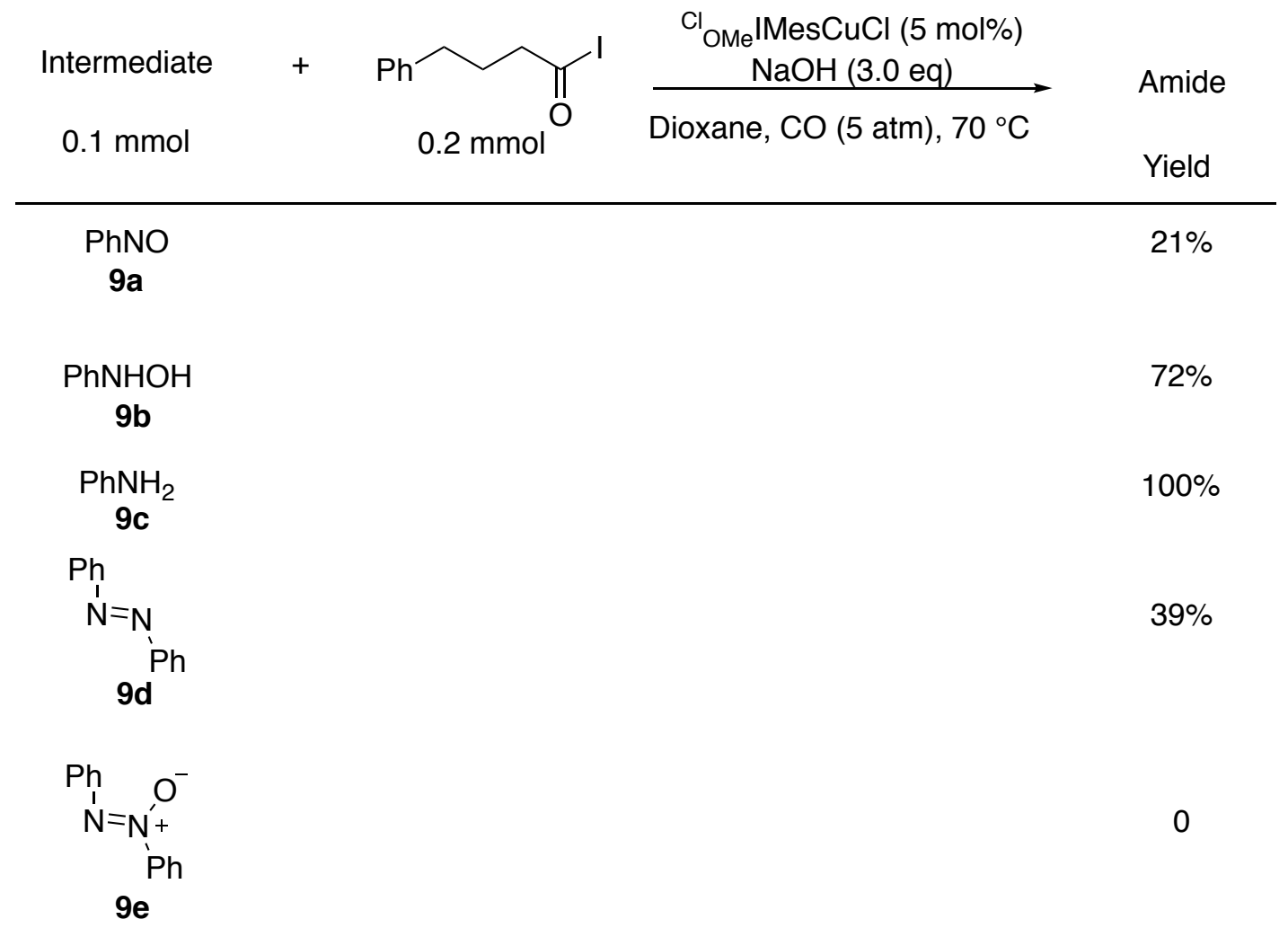

\subsection{Control Experiment without $\mathrm{Cu}$ Catalyst}

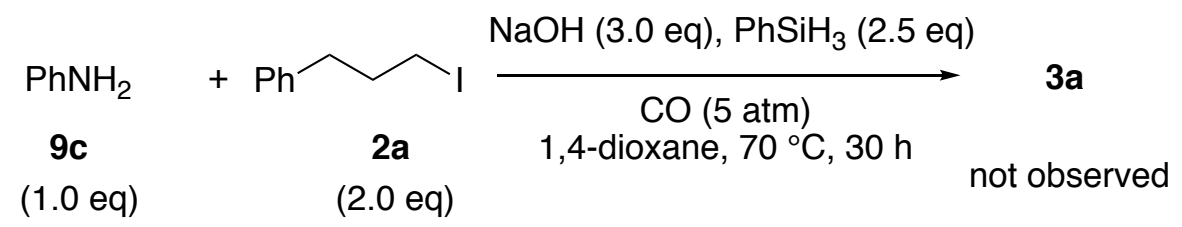

Aniline $(0.1 \mathrm{mmol}, 9 \mu \mathrm{L}, 1.0 \mathrm{eq})$ was added to the reaction shown above without the presence of copper catalyst. The targeted amide compound $\mathbf{3 a}$ was not observed. 


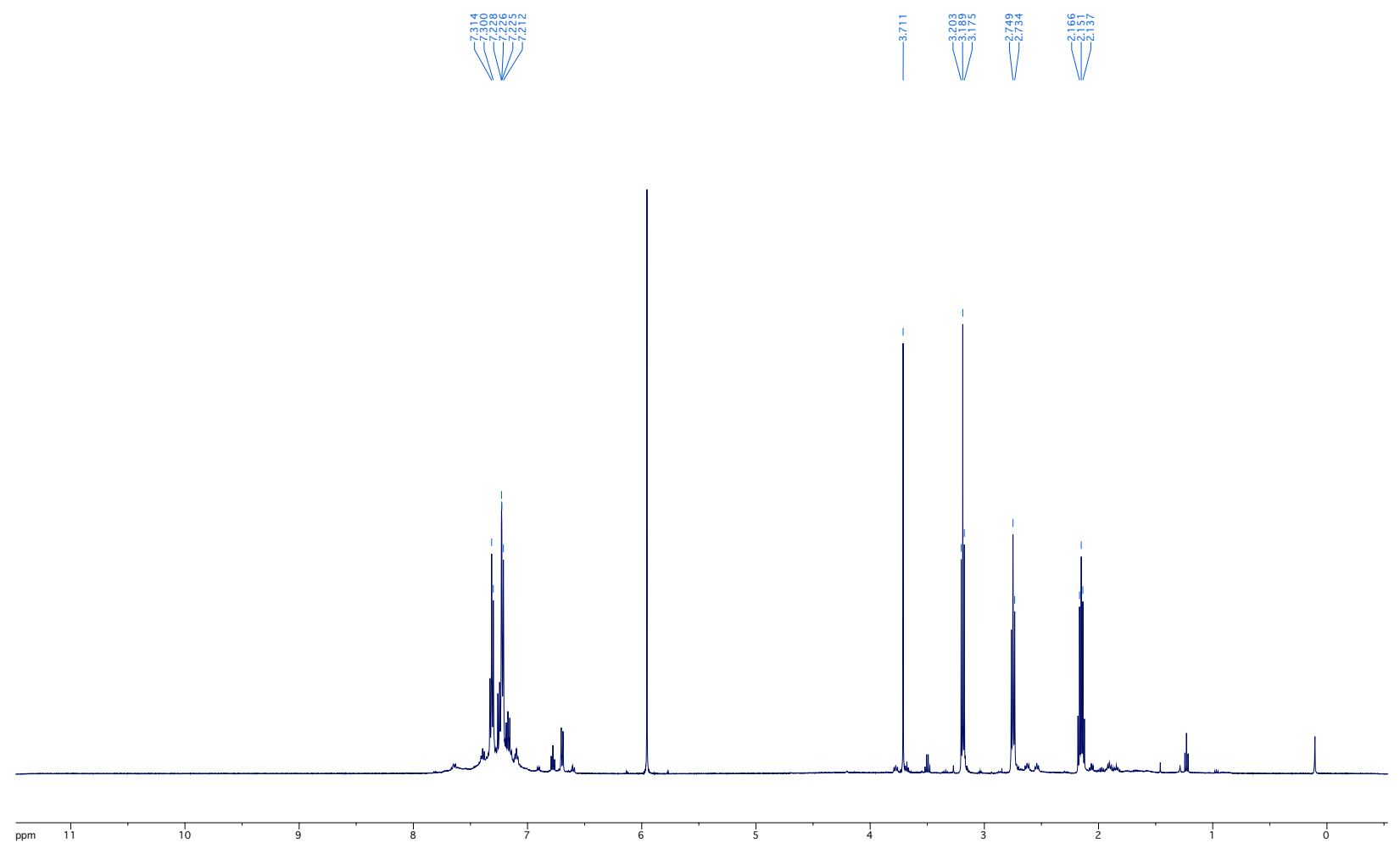

\subsection{Experiment with Potential Copper Amide Intermediate}<smiles>[C+]C(C)CNc1ccccc1</smiles>

10

(1.0 eq)

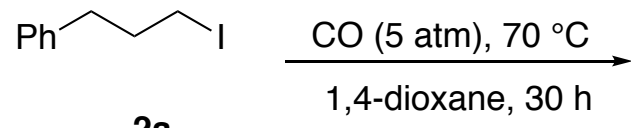

2a

$(1.0 \mathrm{eq})$
$3 a$

not observed

${ }^{\mathrm{Cl}} \mathrm{IMes}$ copper phenyl amide ( $\left.0.028 \mathrm{mmol}, 0.015 \mathrm{~g}, 1.0 \mathrm{eq}\right)$ was subjected to 3-phenyl-1-iodopropane (2a) $(0.028 \mathrm{mmol}, 5 \mu \mathrm{L}, 1.0 \mathrm{eq}) \mathrm{in} 1 \mathrm{ml}$ dioxane, and let the mixture stir for 30 hours at $70{ }^{\circ} \mathrm{C}$ and under $5 \mathrm{~atm}$ carbon monoxide. However, there was no formation of amide compound 3a. 


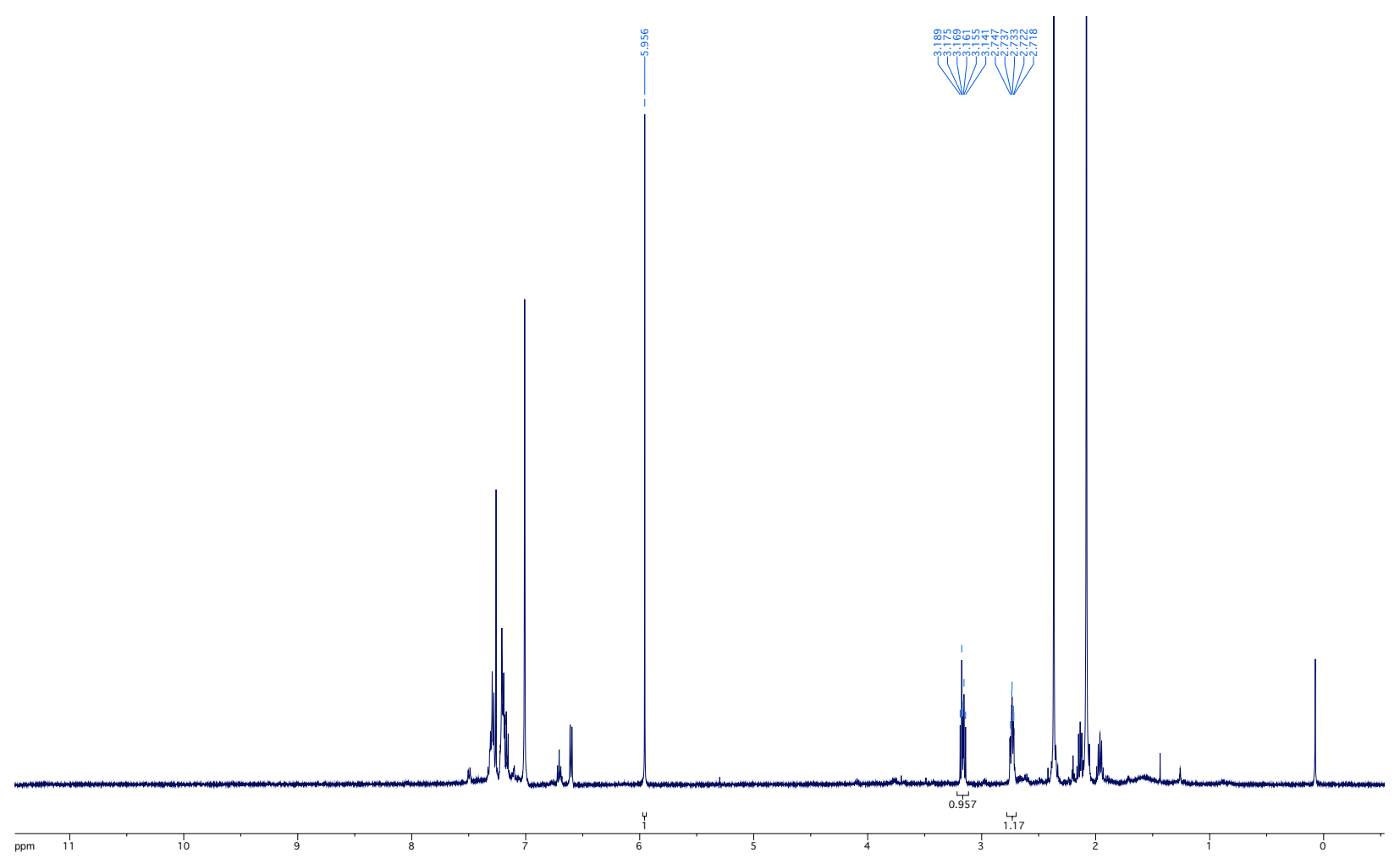


5. ${ }^{1} \mathrm{H}$ and ${ }^{13} \mathrm{C}$ Spectra

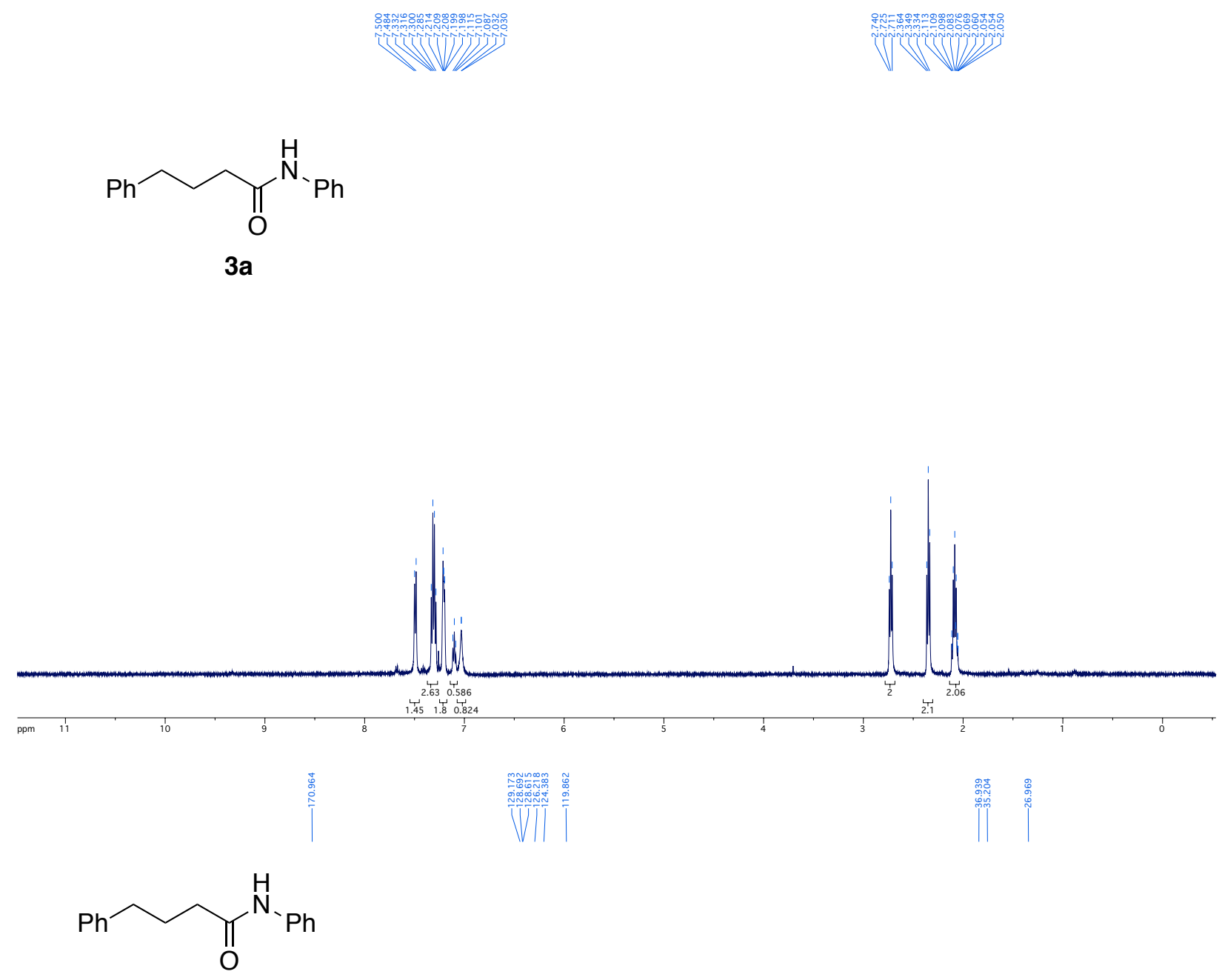

3a

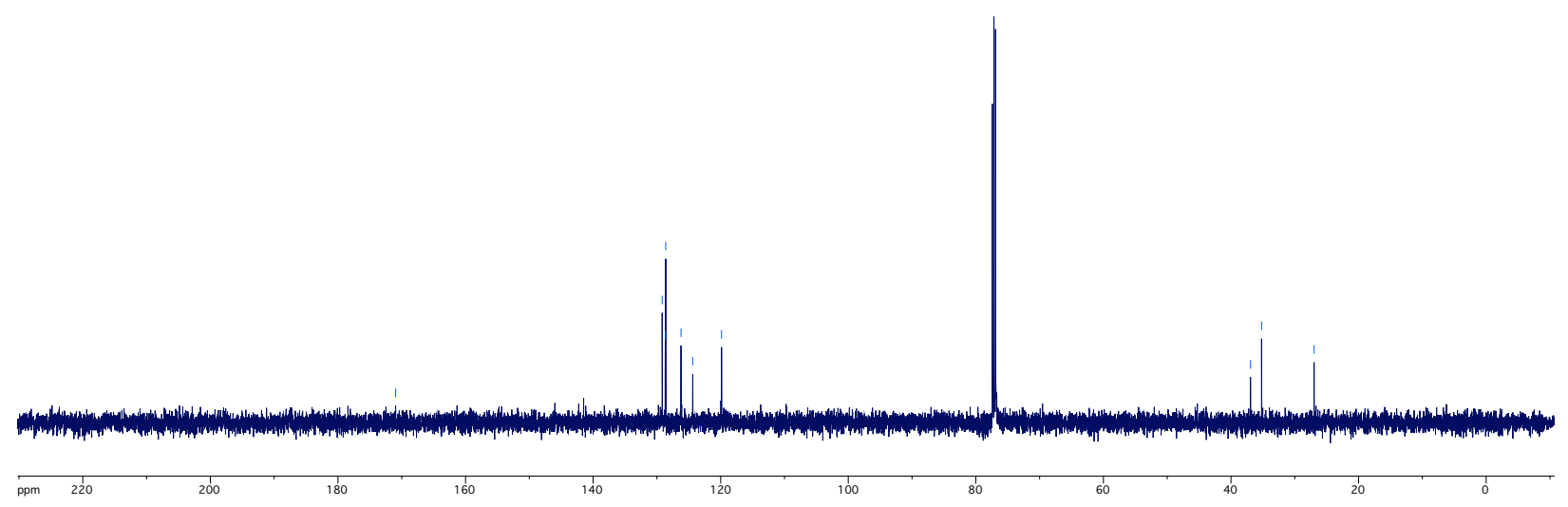




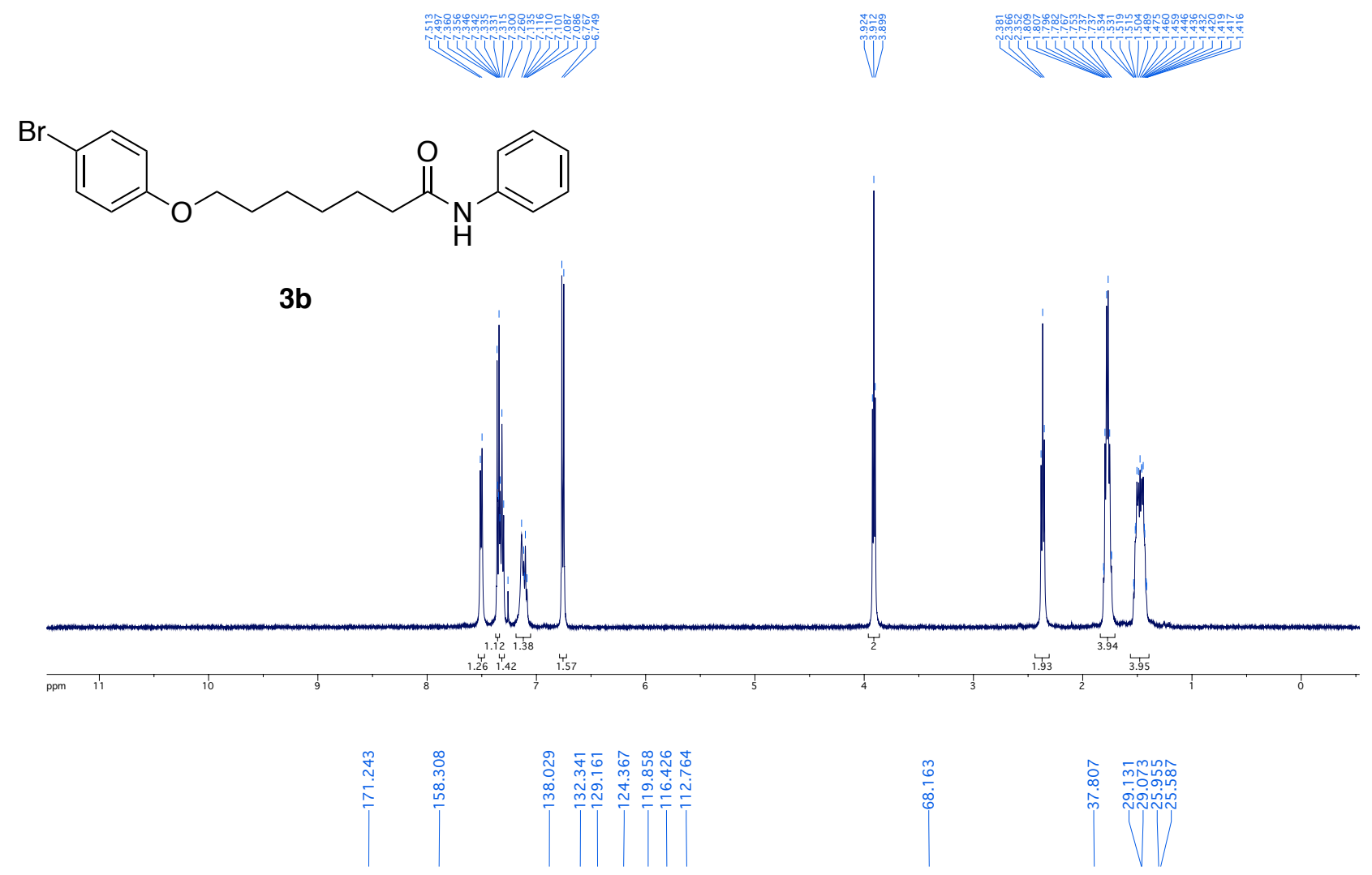

(N)

$3 b$

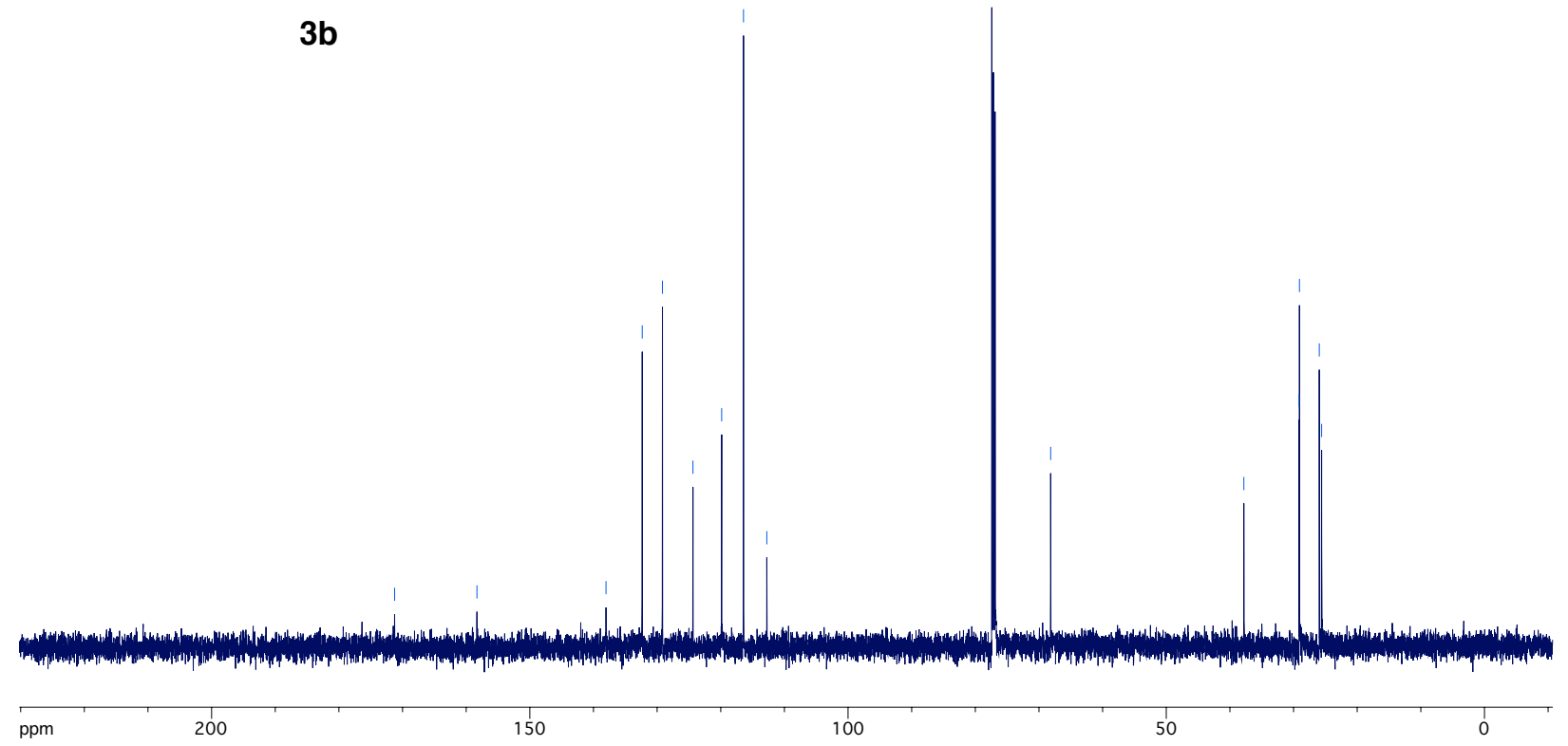



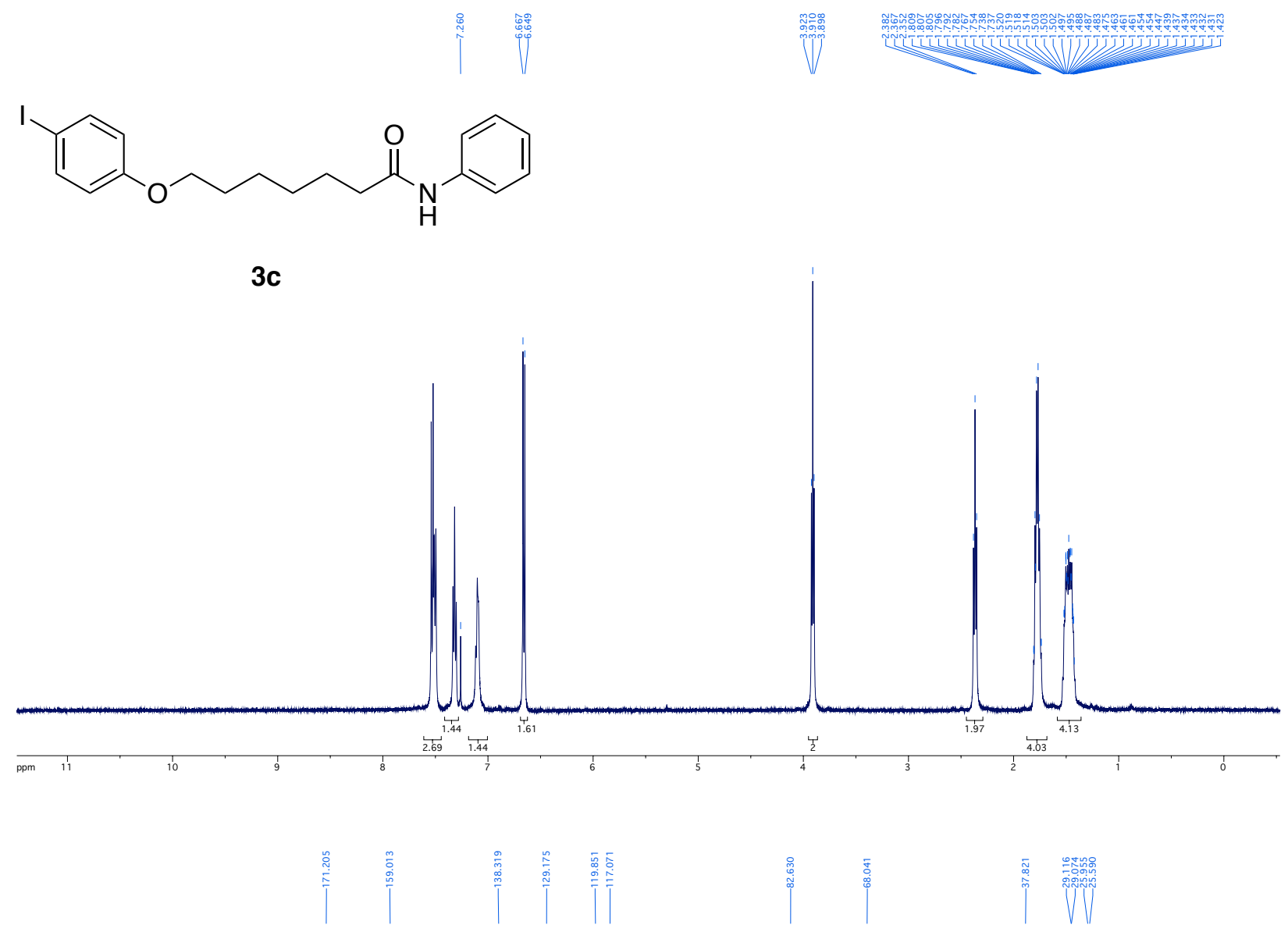<smiles>O=C(CCCCCCOc1ccc(I)cc1)Nc1ccccc1</smiles>

$3 c$

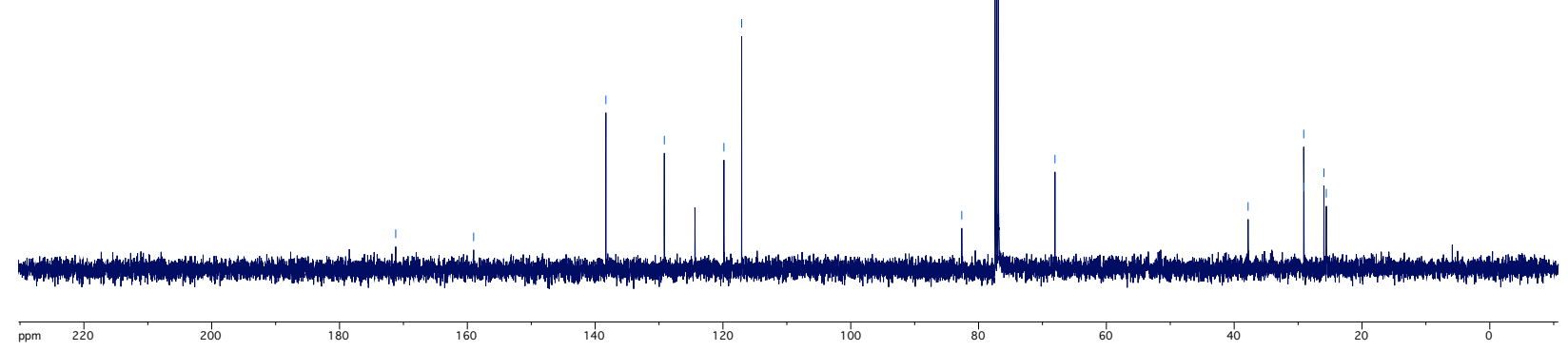


(c)

3d
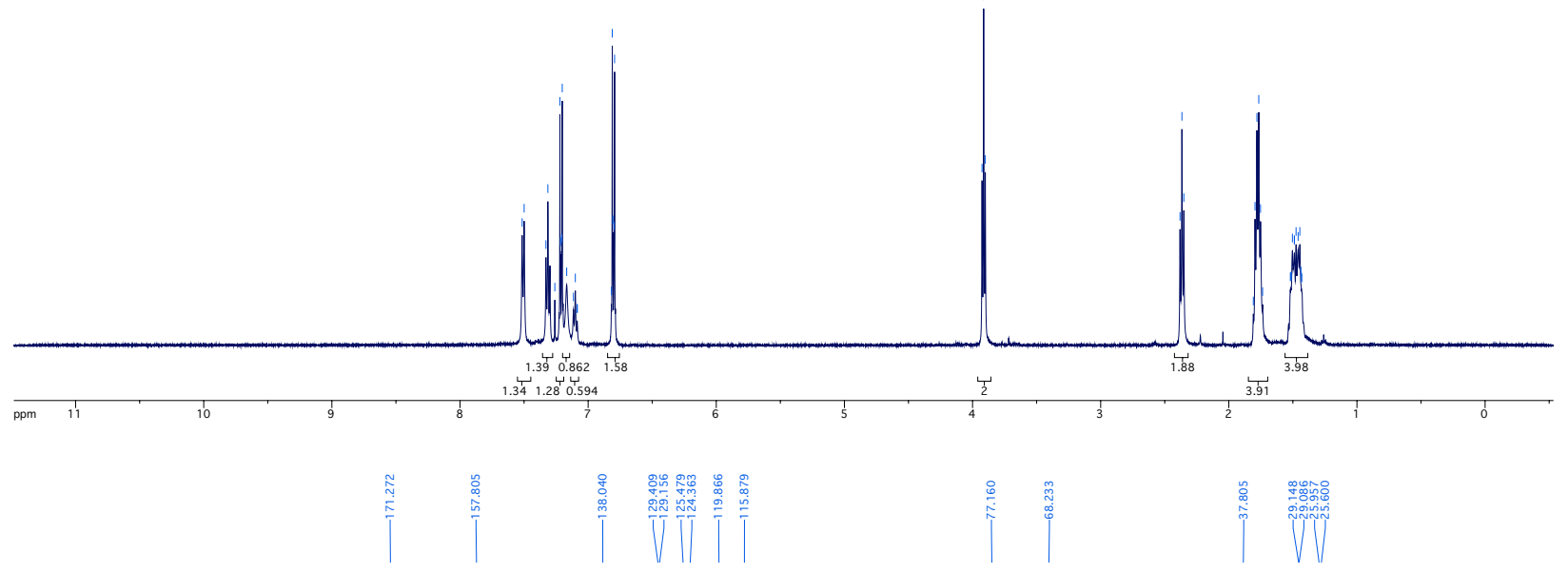<smiles>O=C(CCCCCCOc1ccc(Cl)cc1)Nc1ccccc1</smiles>

3d

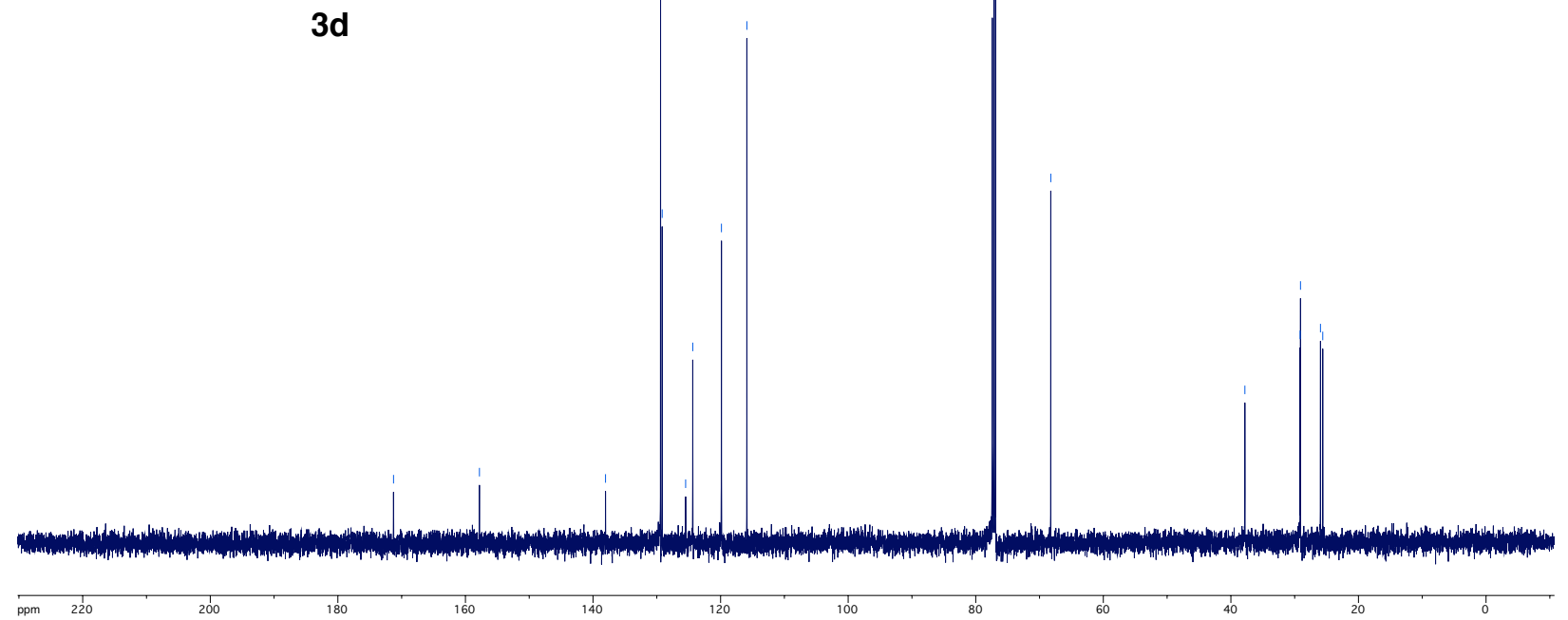




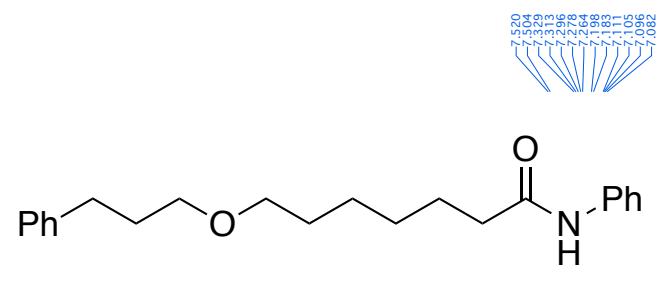

$3 e$
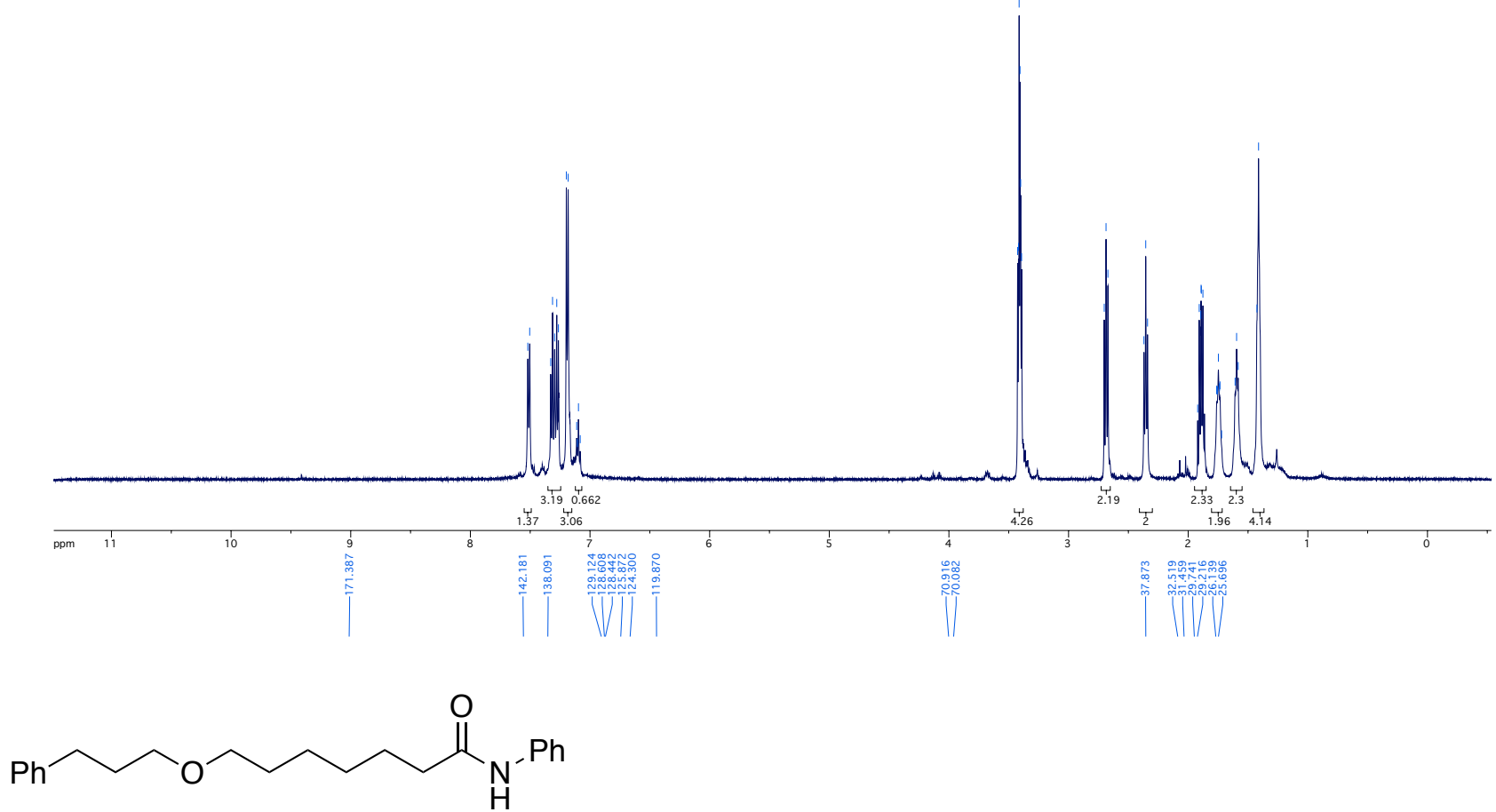

$3 e$

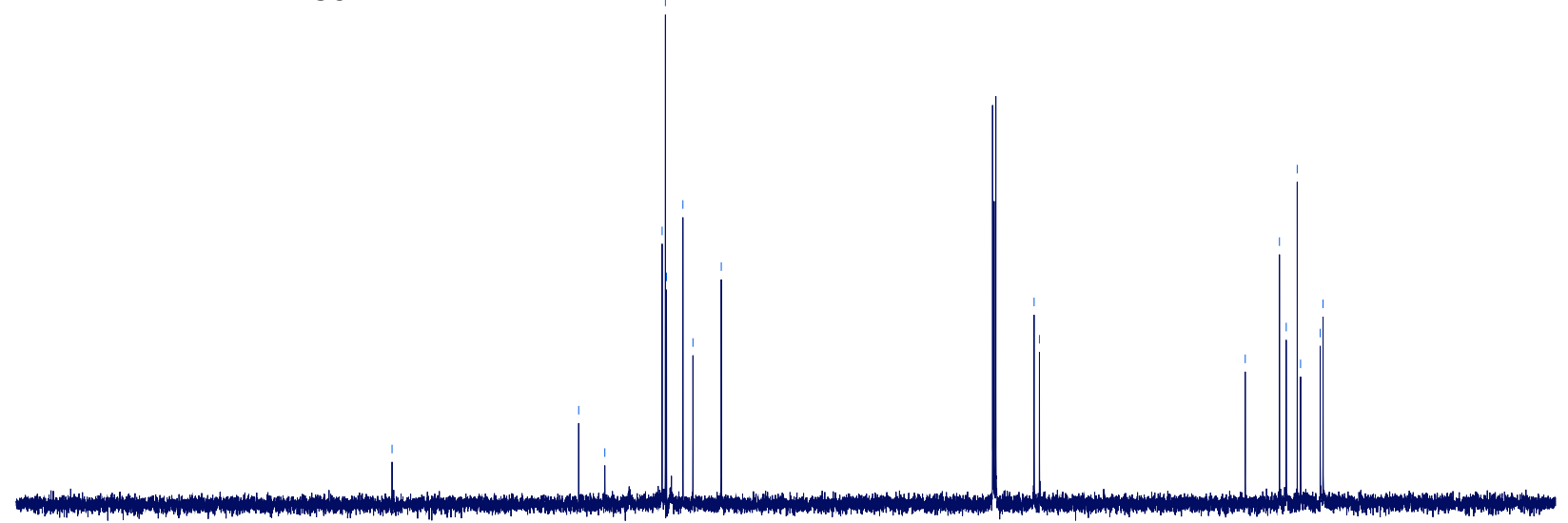

ppm 22 180 140 80 

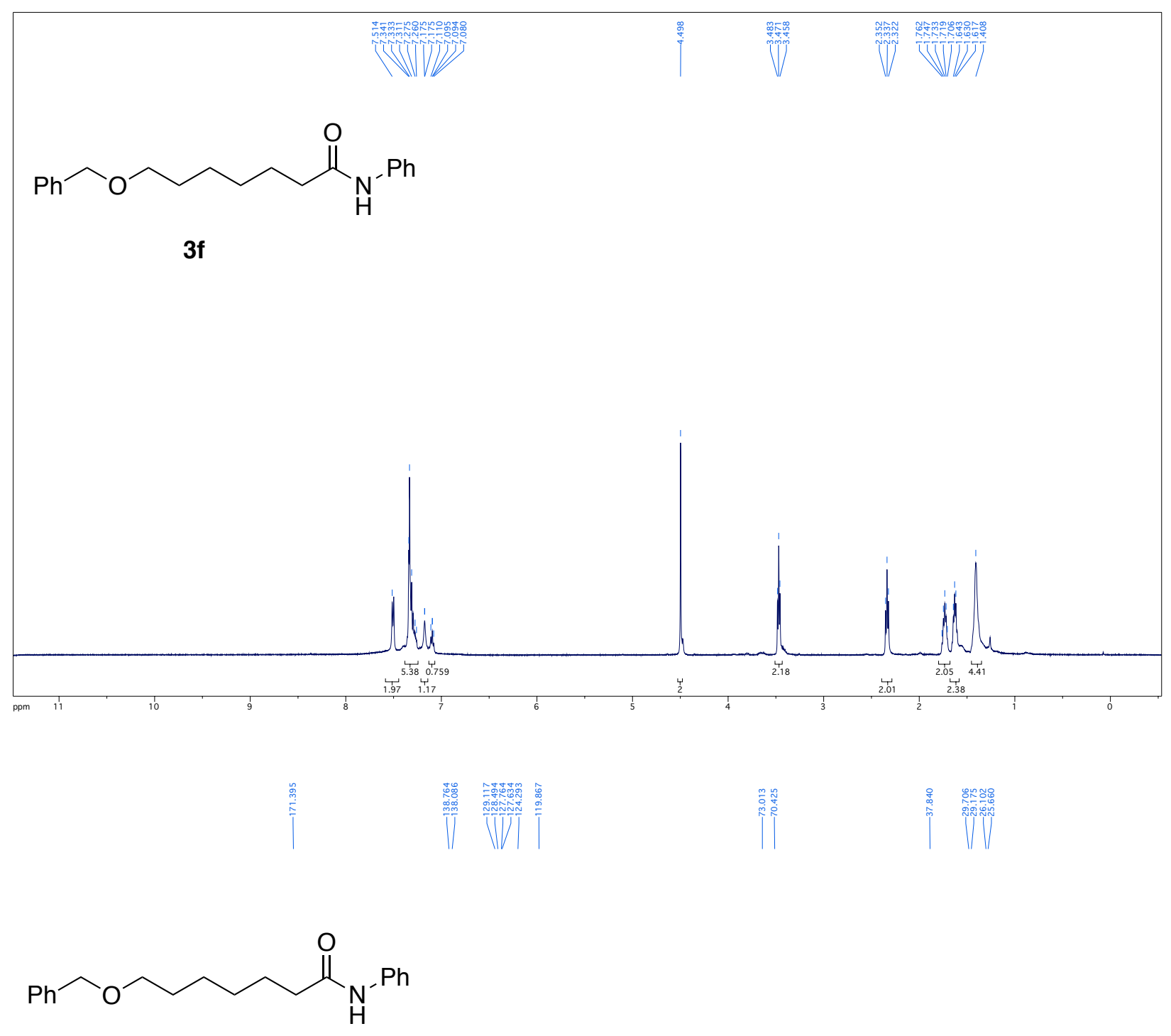

$3 f$

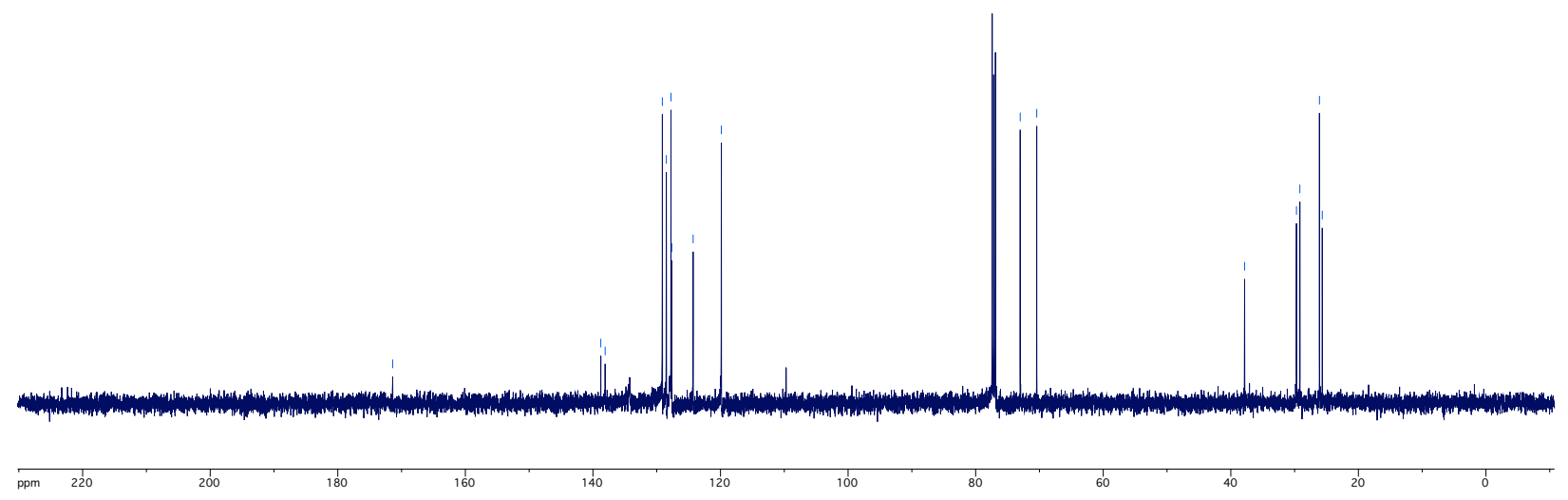


$\mathrm{C}_{\mathrm{H}^{-}}^{\mathrm{PCh}}$

$3 g$
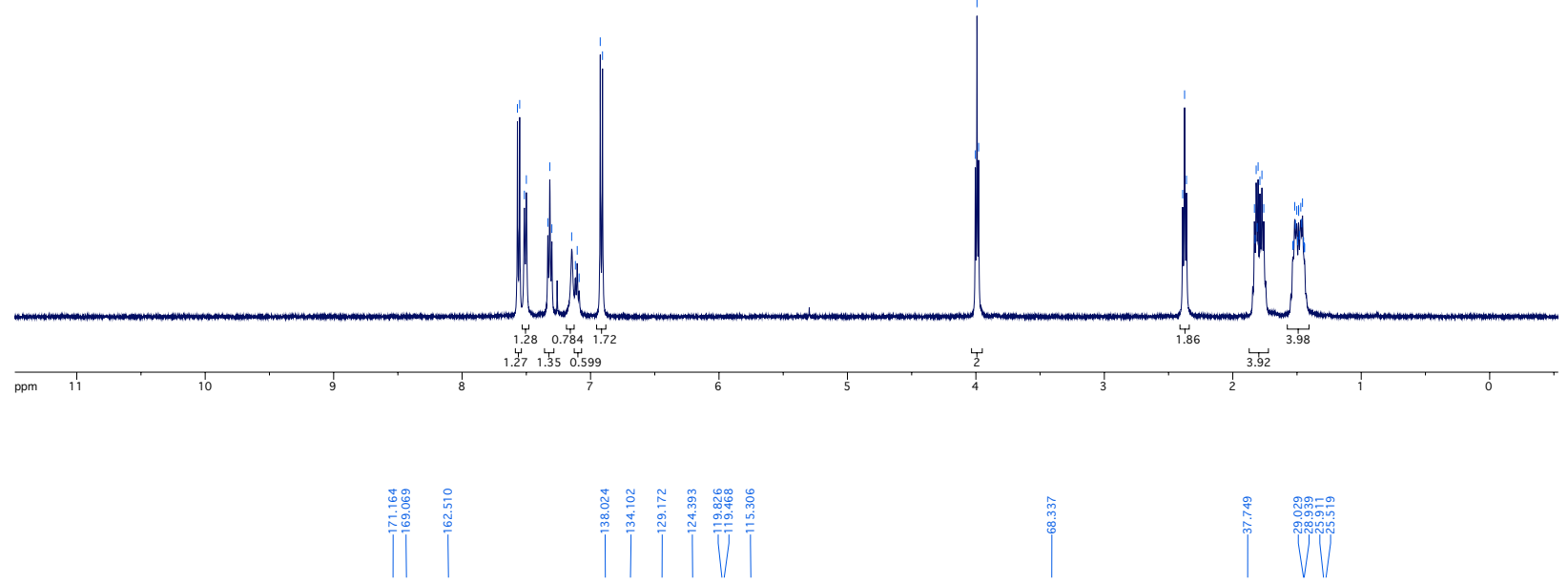<smiles>N#Cc1ccc(OCCCCCCC(=O)Nc2ccccc2)cc1</smiles>

$3 g$

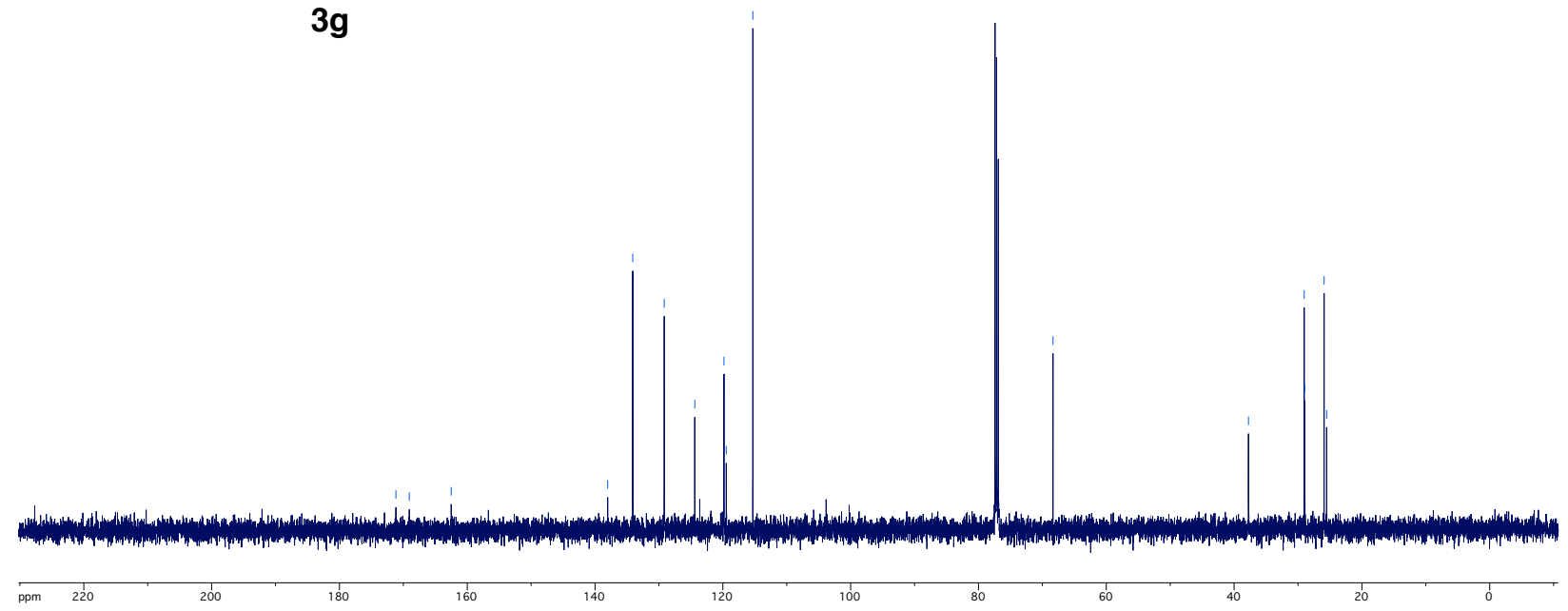



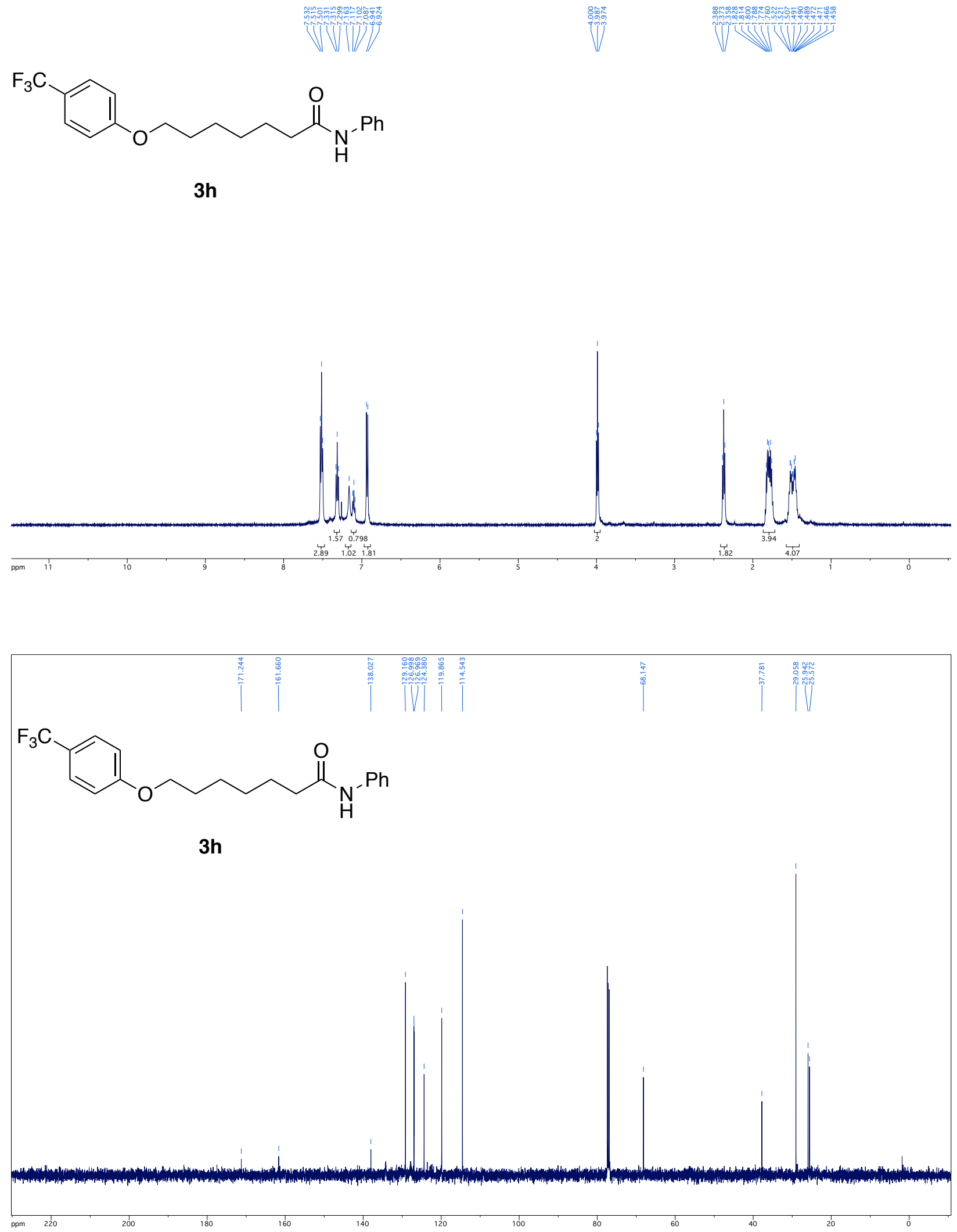


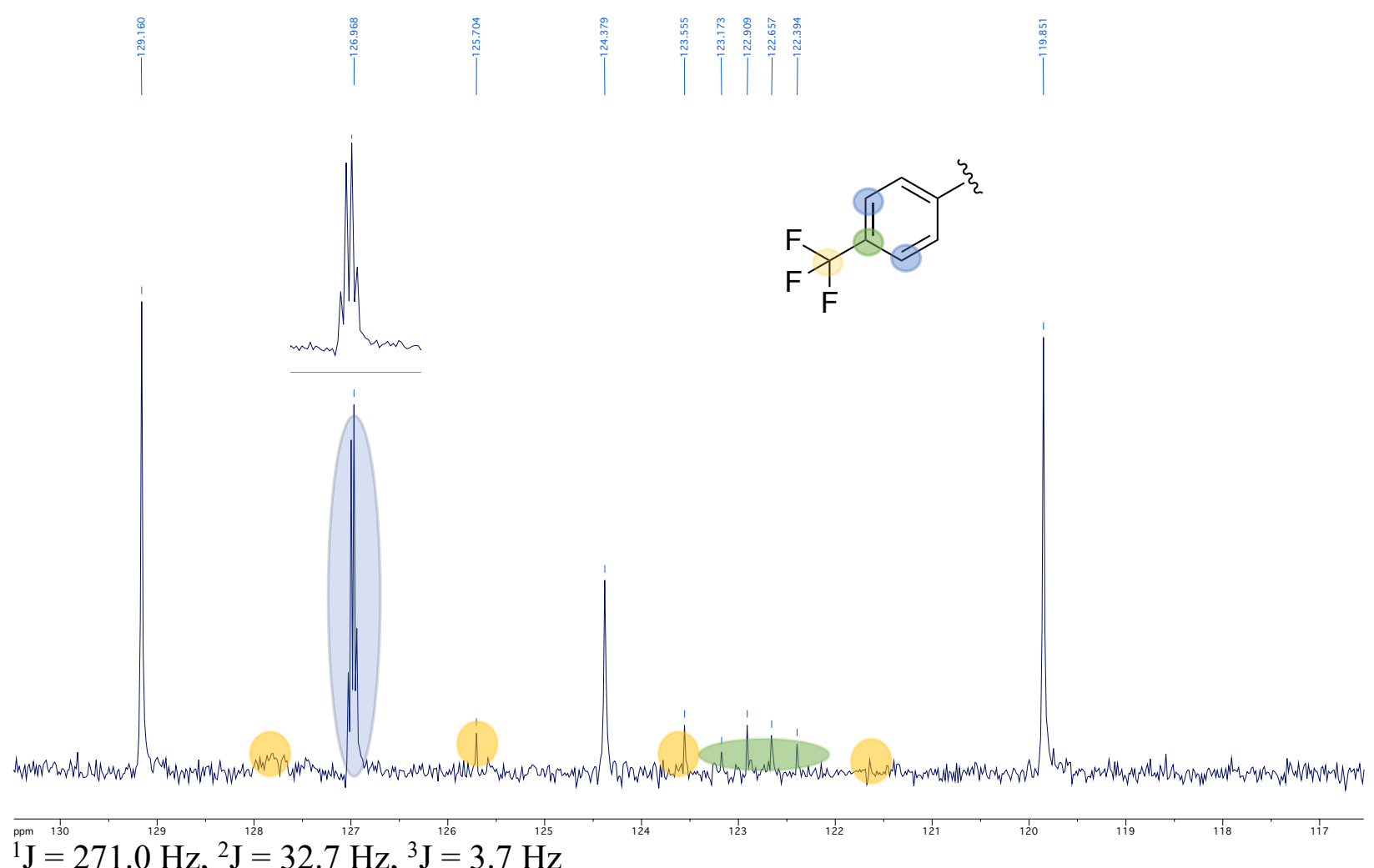

${ }^{1} \mathrm{~J}=271.0 \mathrm{~Hz},{ }^{2} \mathrm{~J}=32.7 \mathrm{~Hz},{ }^{3} \mathrm{~J}=3.7 \mathrm{~Hz}$ 

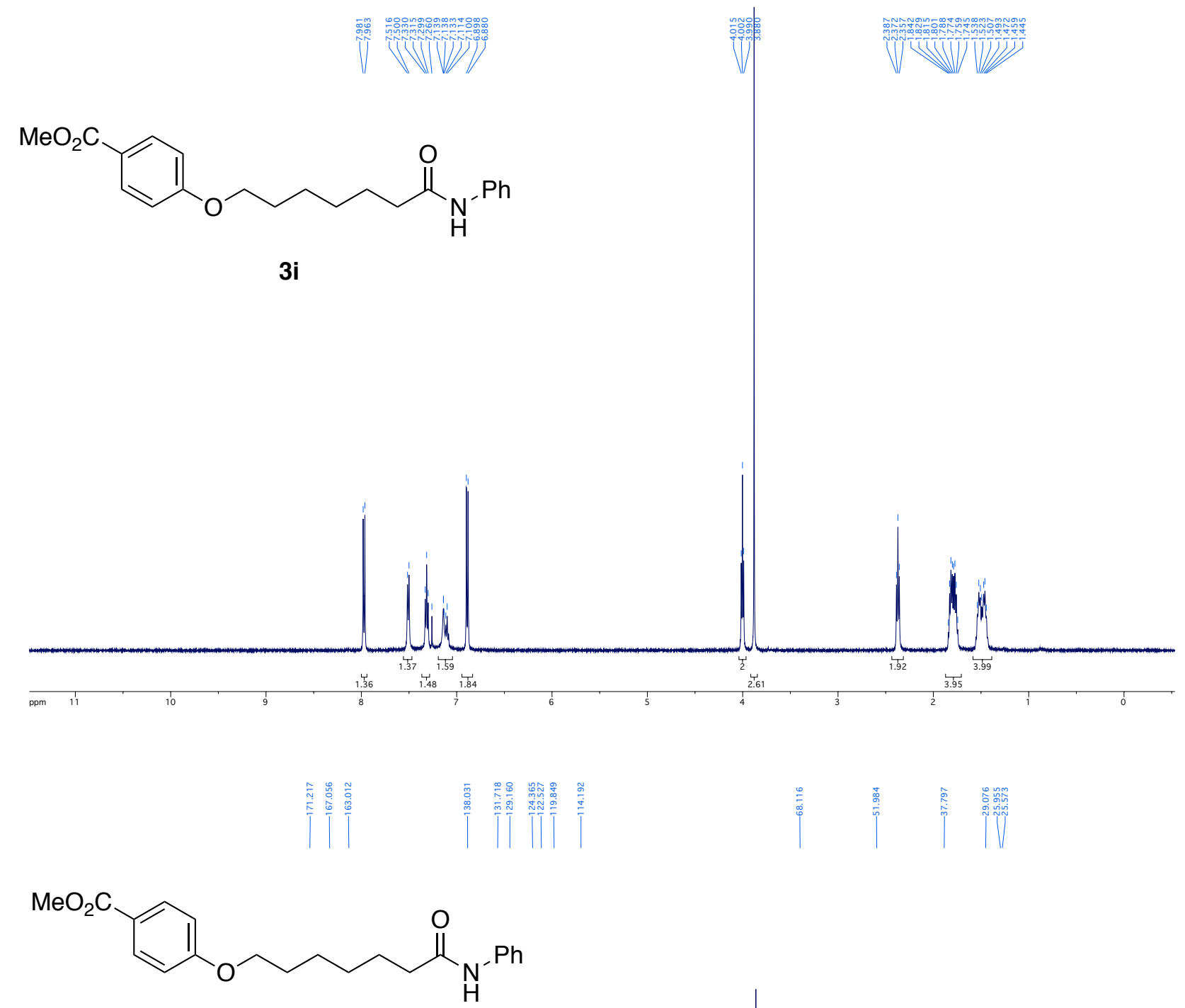

$3 \mathbf{i}$

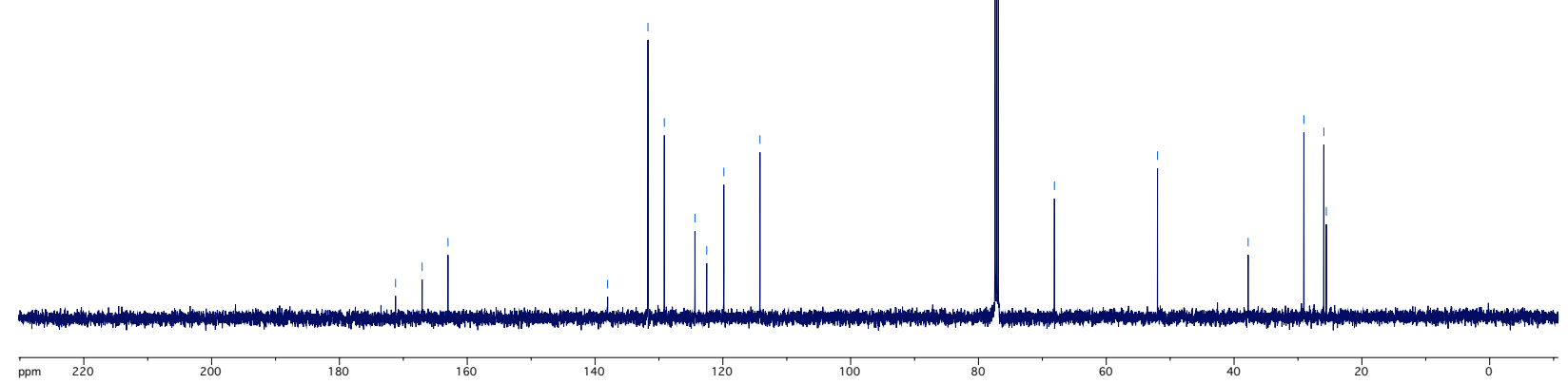




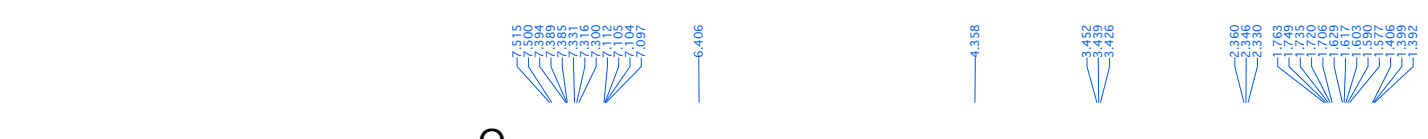<smiles>O=C(CCCCCCOCc1ccco1)Nc1ccccc1</smiles>

3j
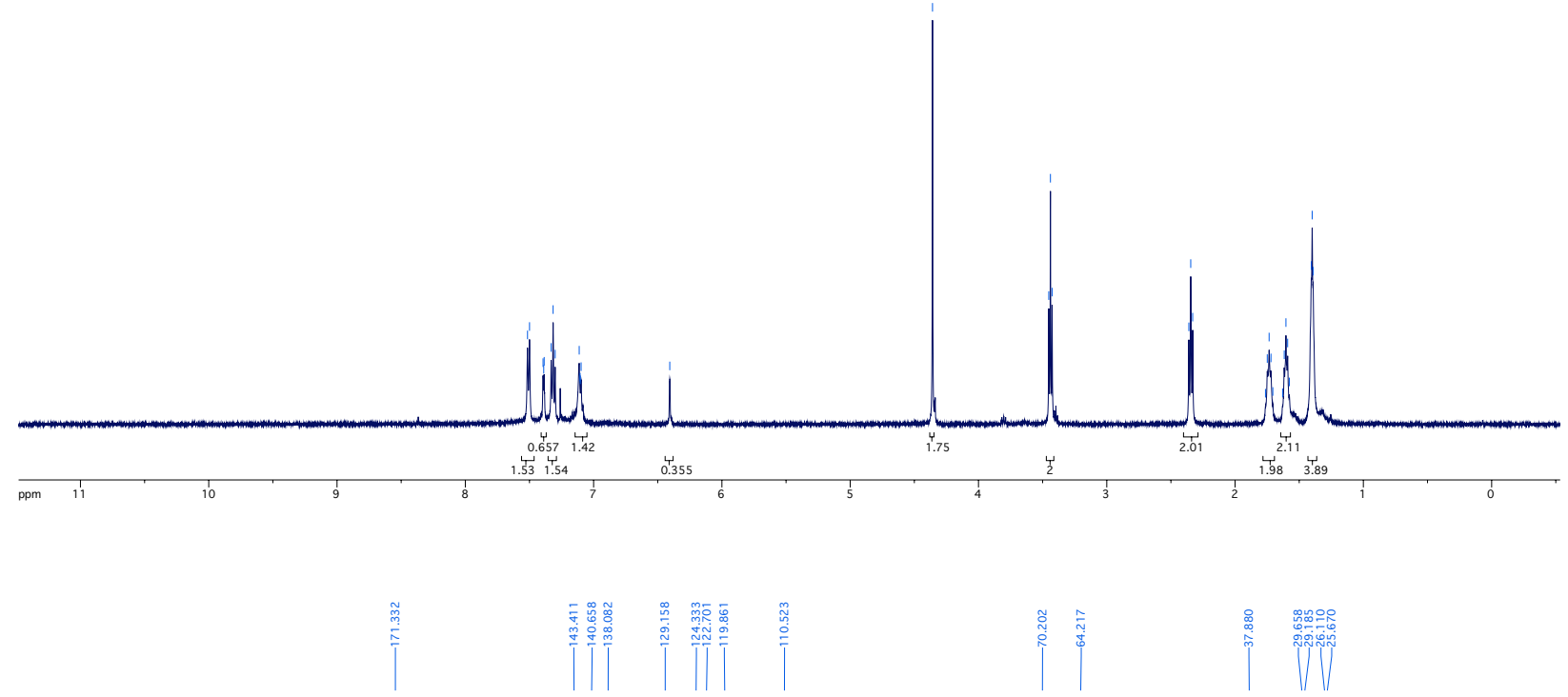<smiles>O=C(CCCCCCOCc1ccco1)Nc1ccccc1</smiles>

3j 

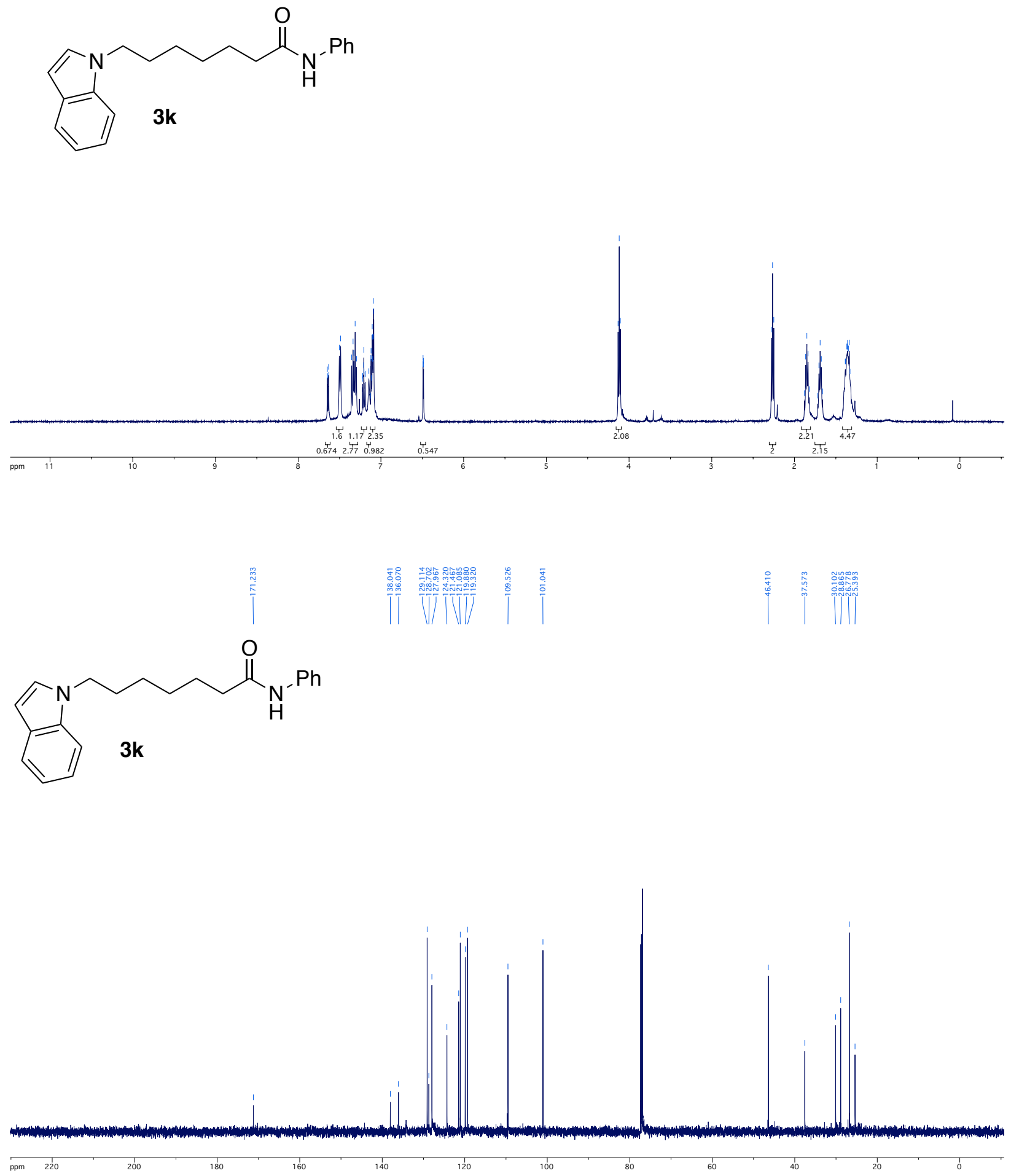
<smiles>O=C(Nc1ccccc1)C1CCCCC1</smiles>

3I

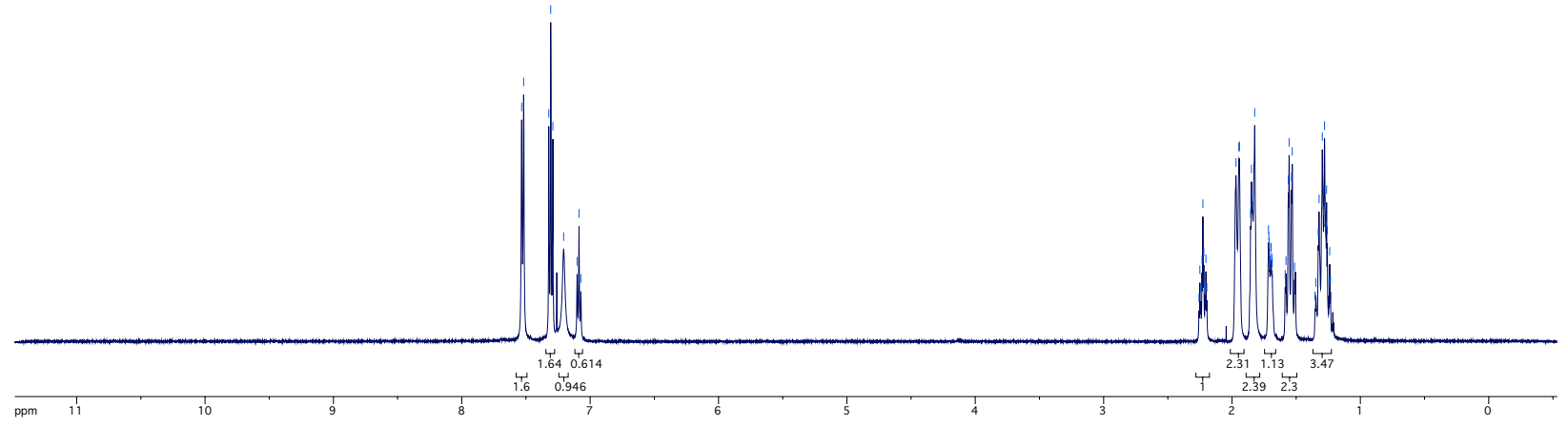<smiles>O=C(Nc1ccccc1)C1CCCCC1</smiles>

3!

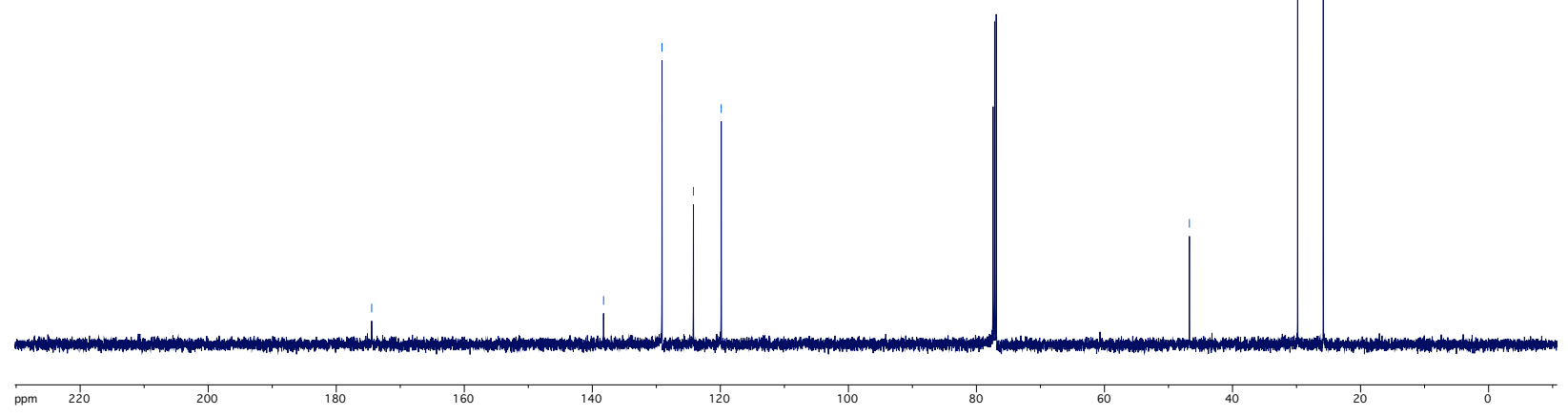




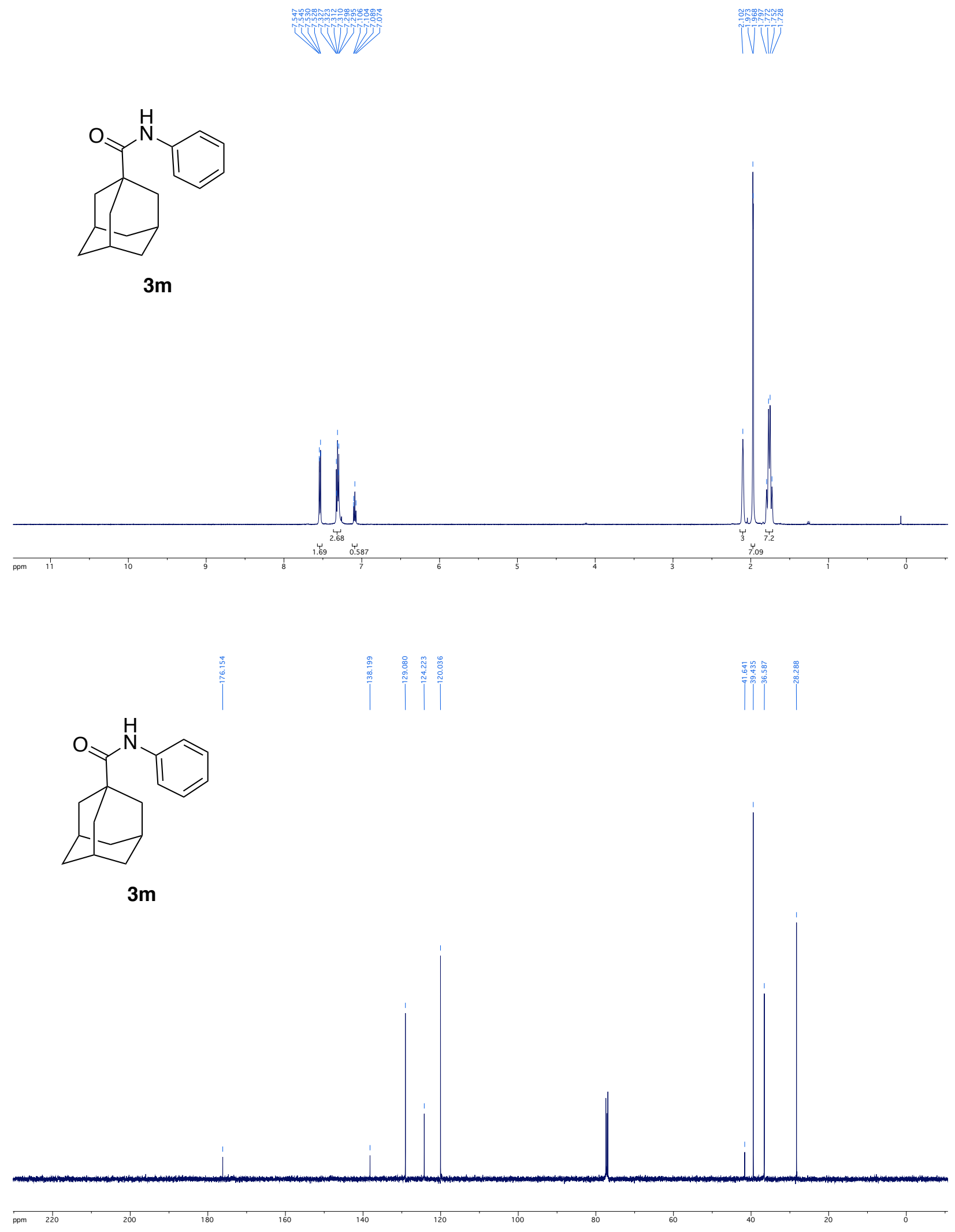




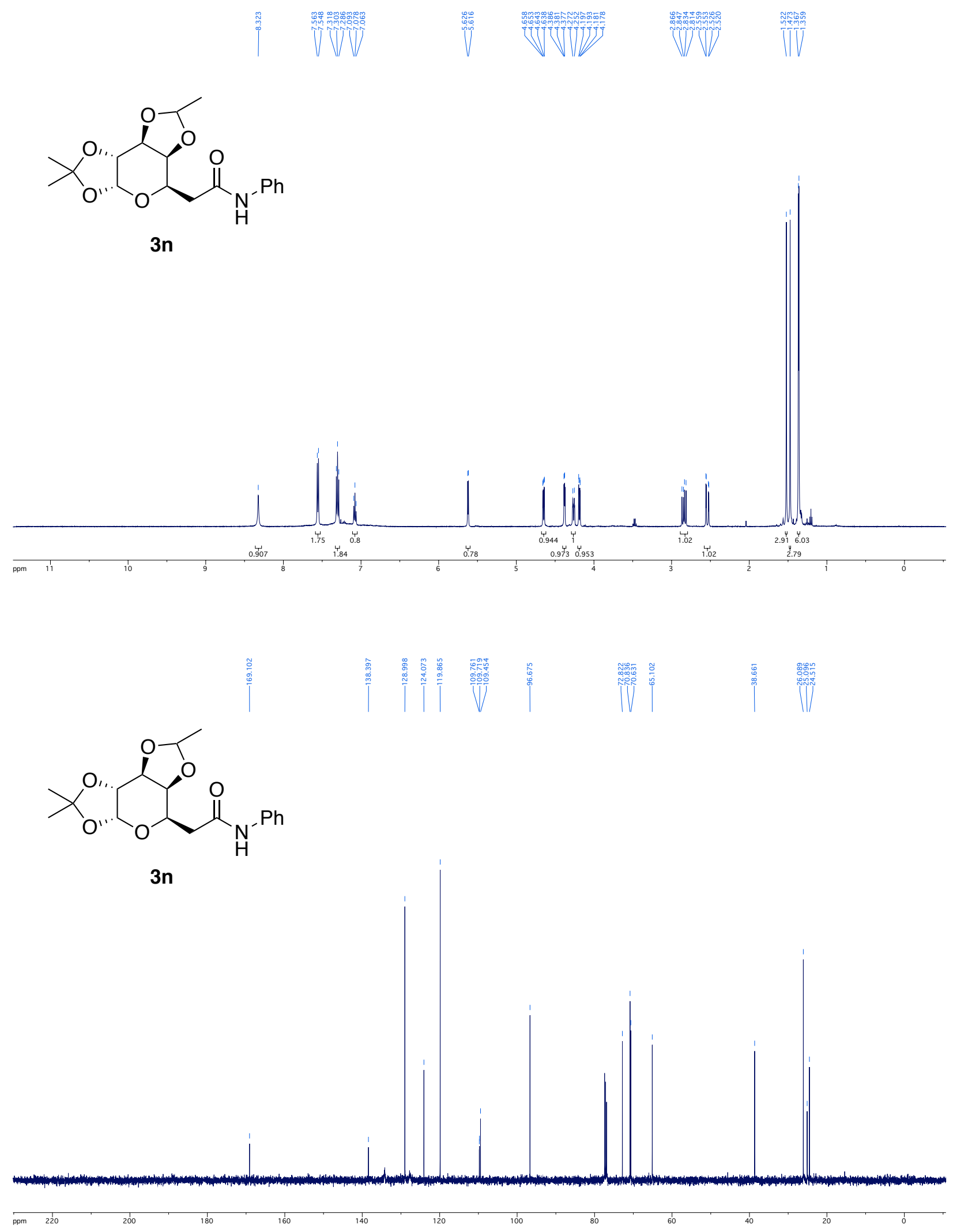



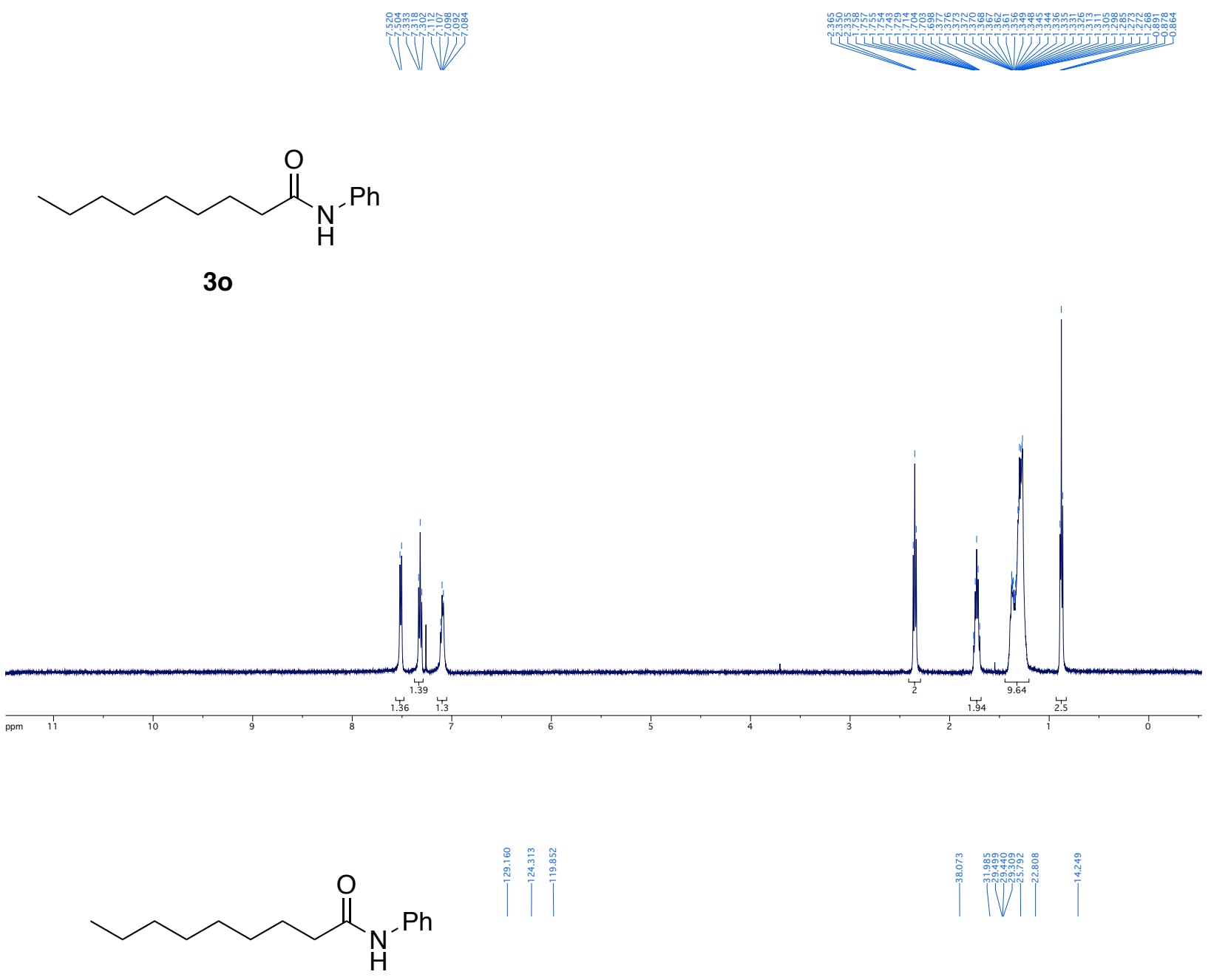

30

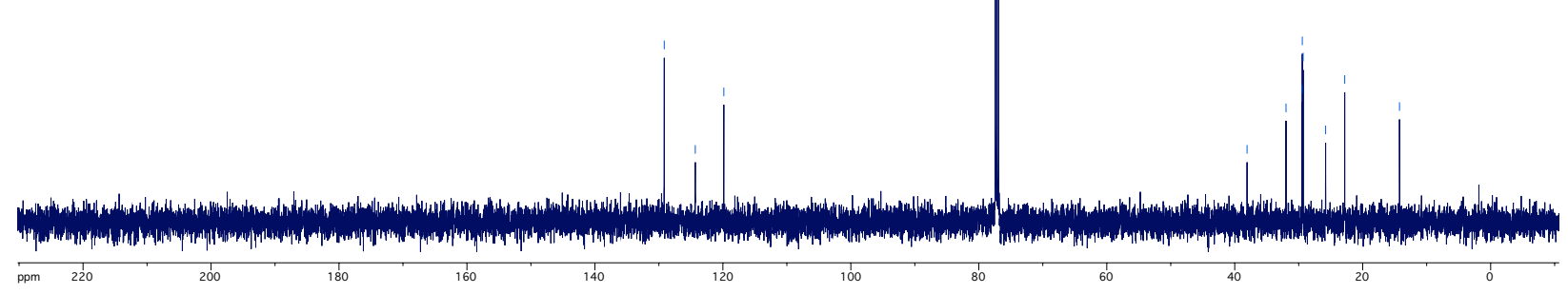




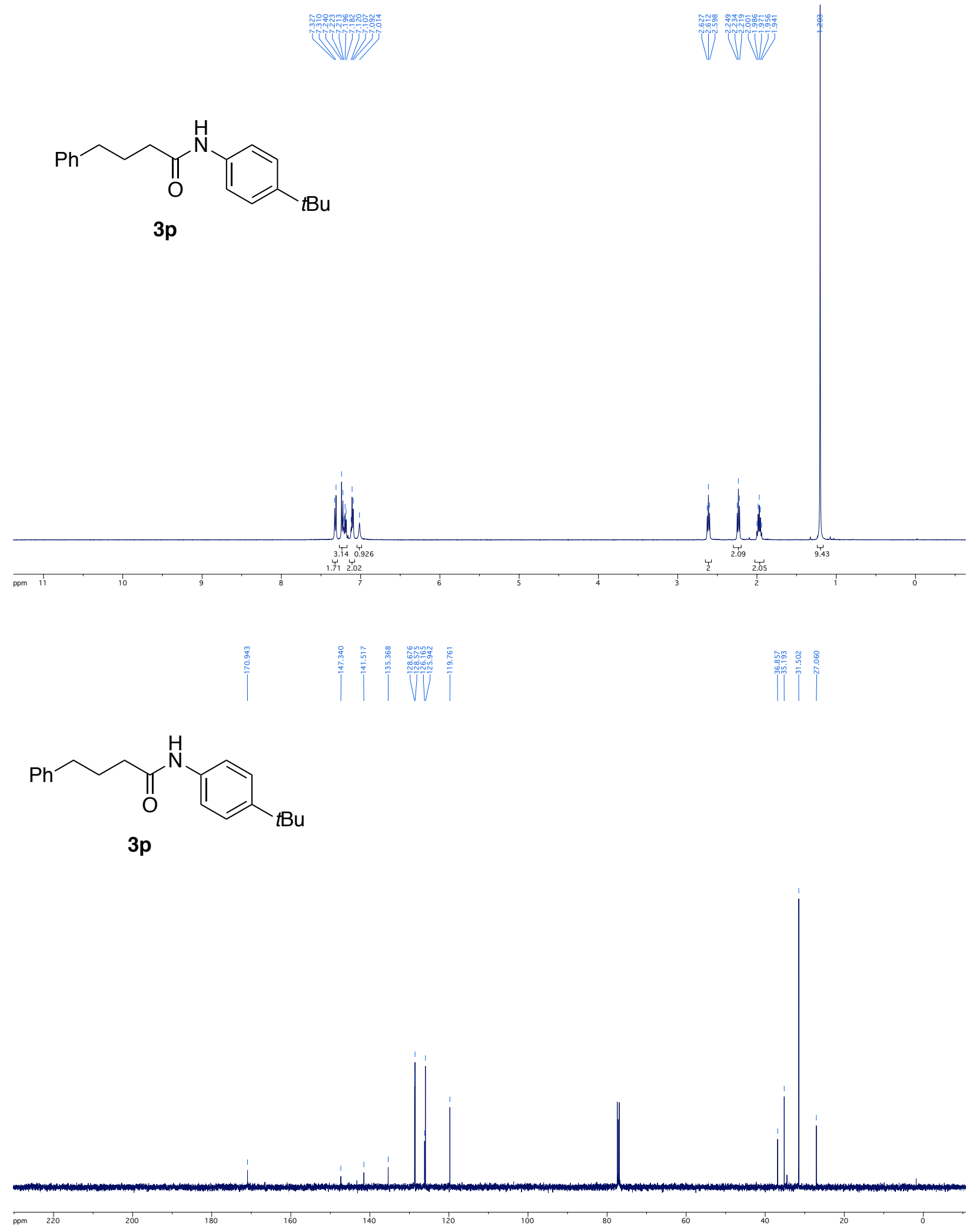



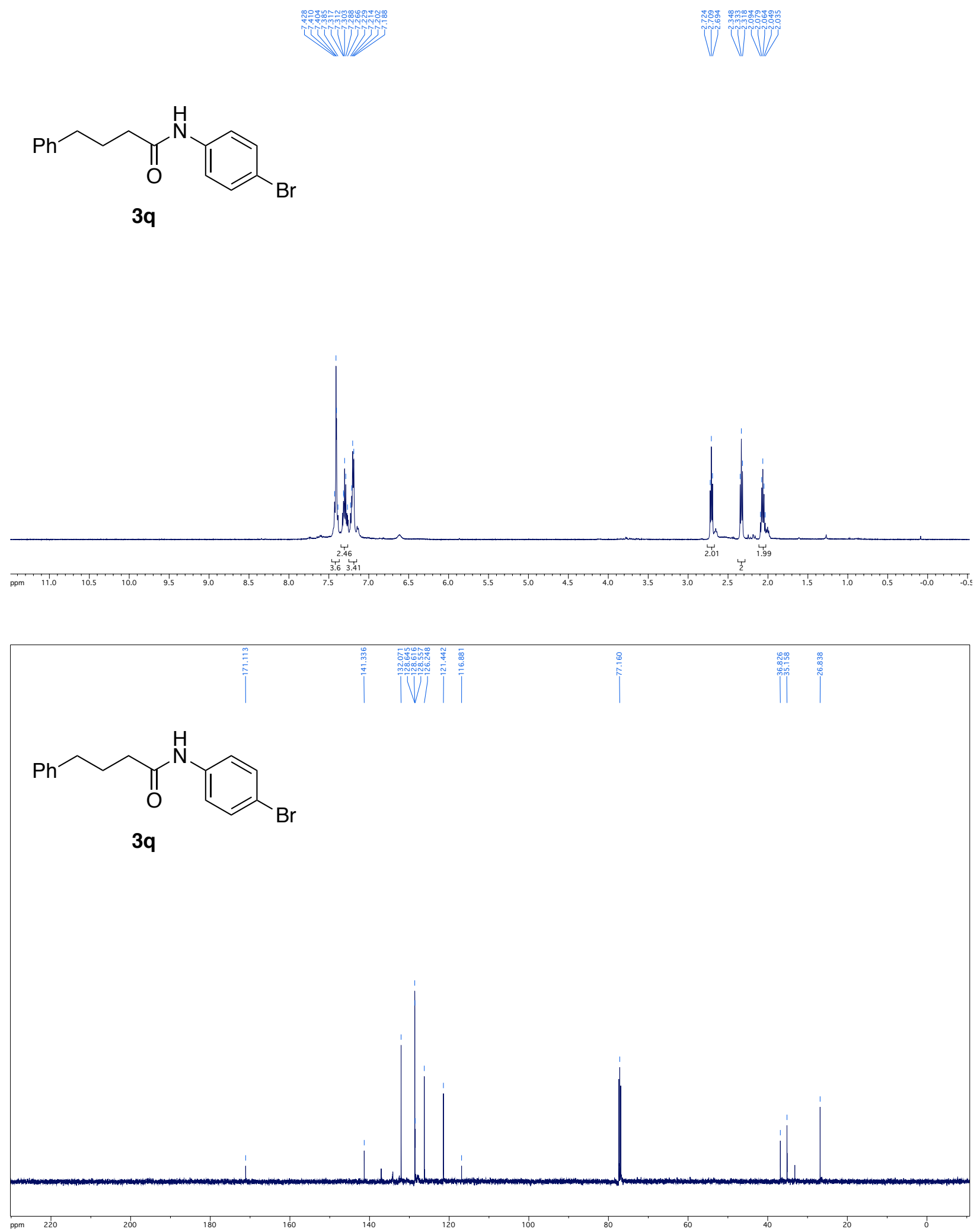

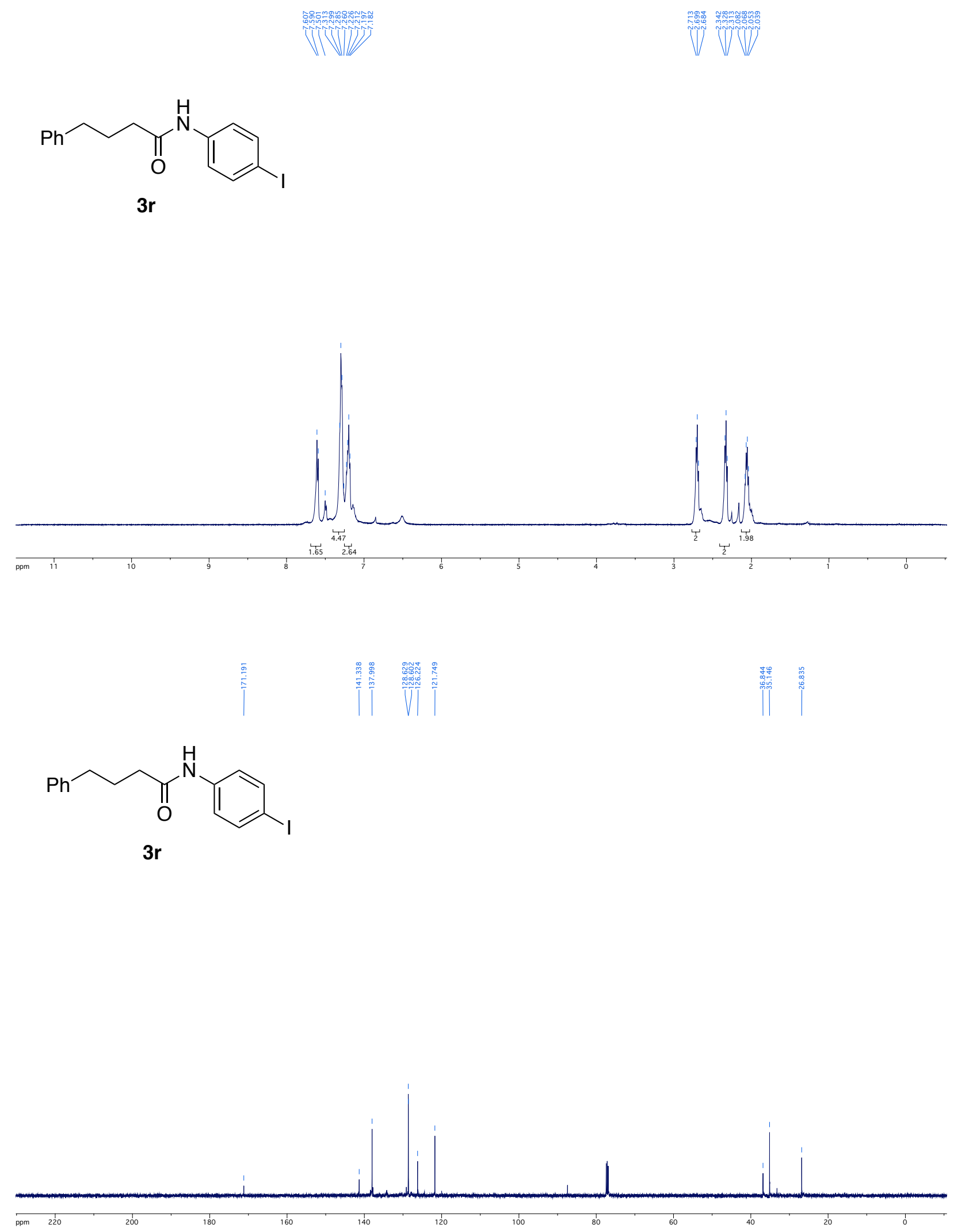


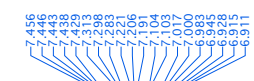

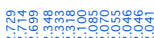

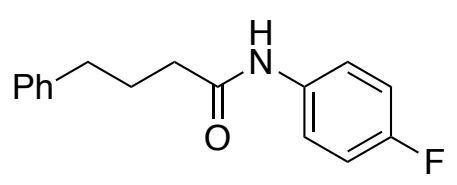

3s
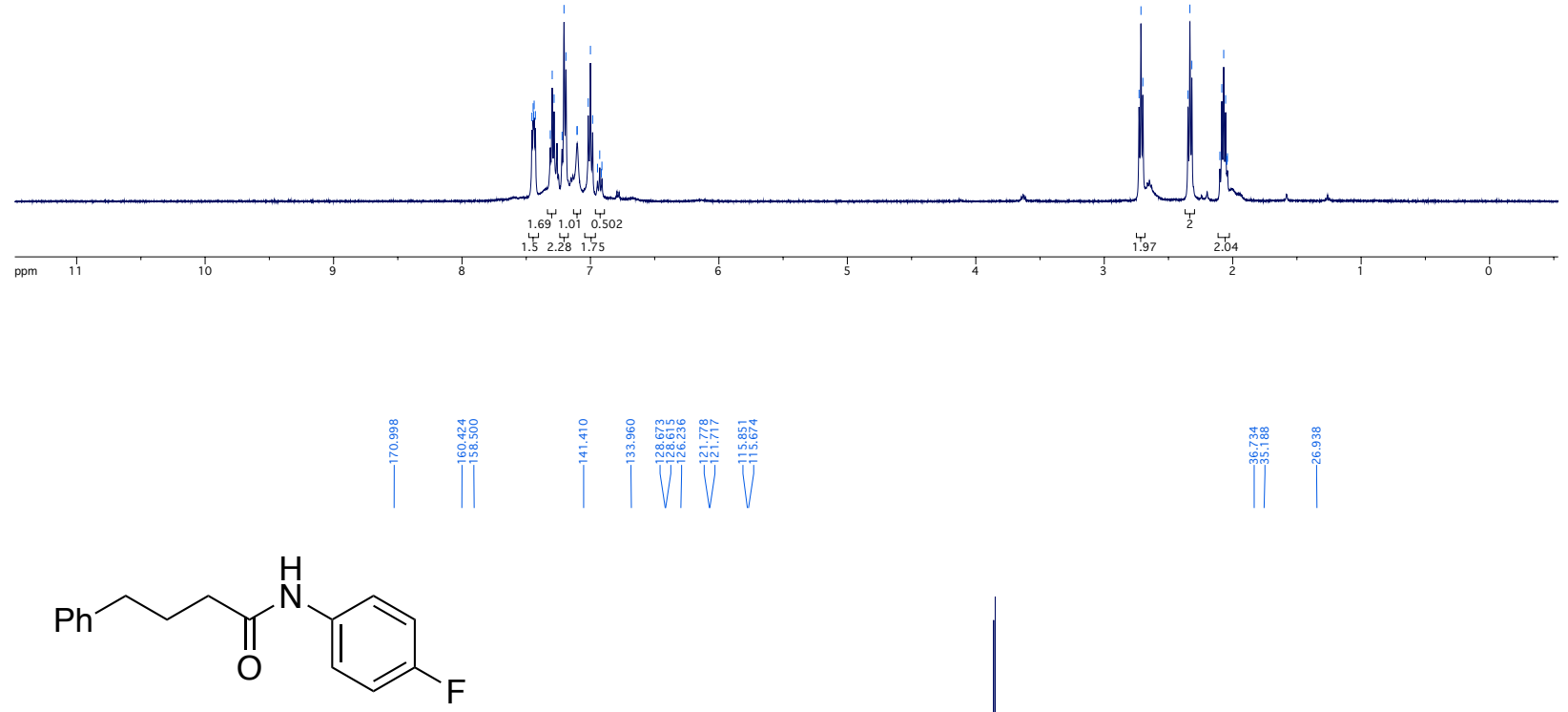

$3 s$

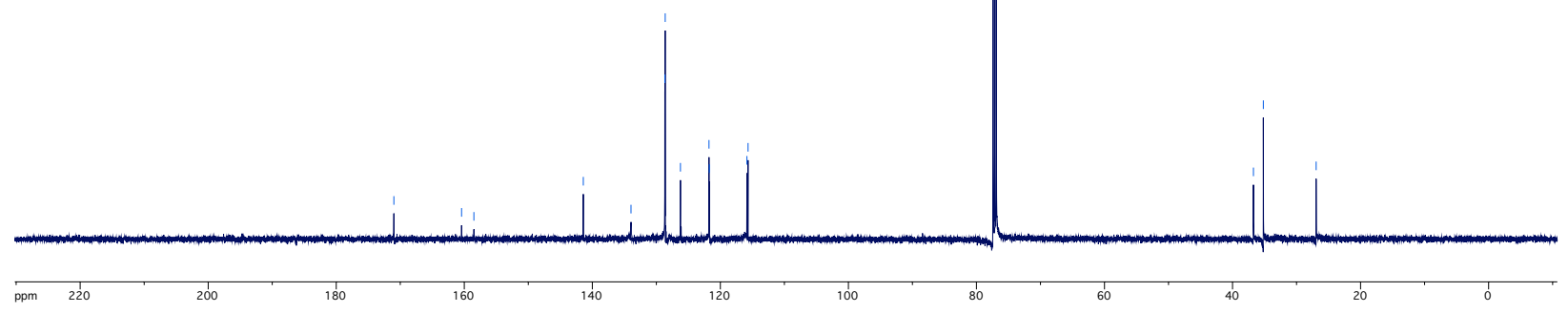



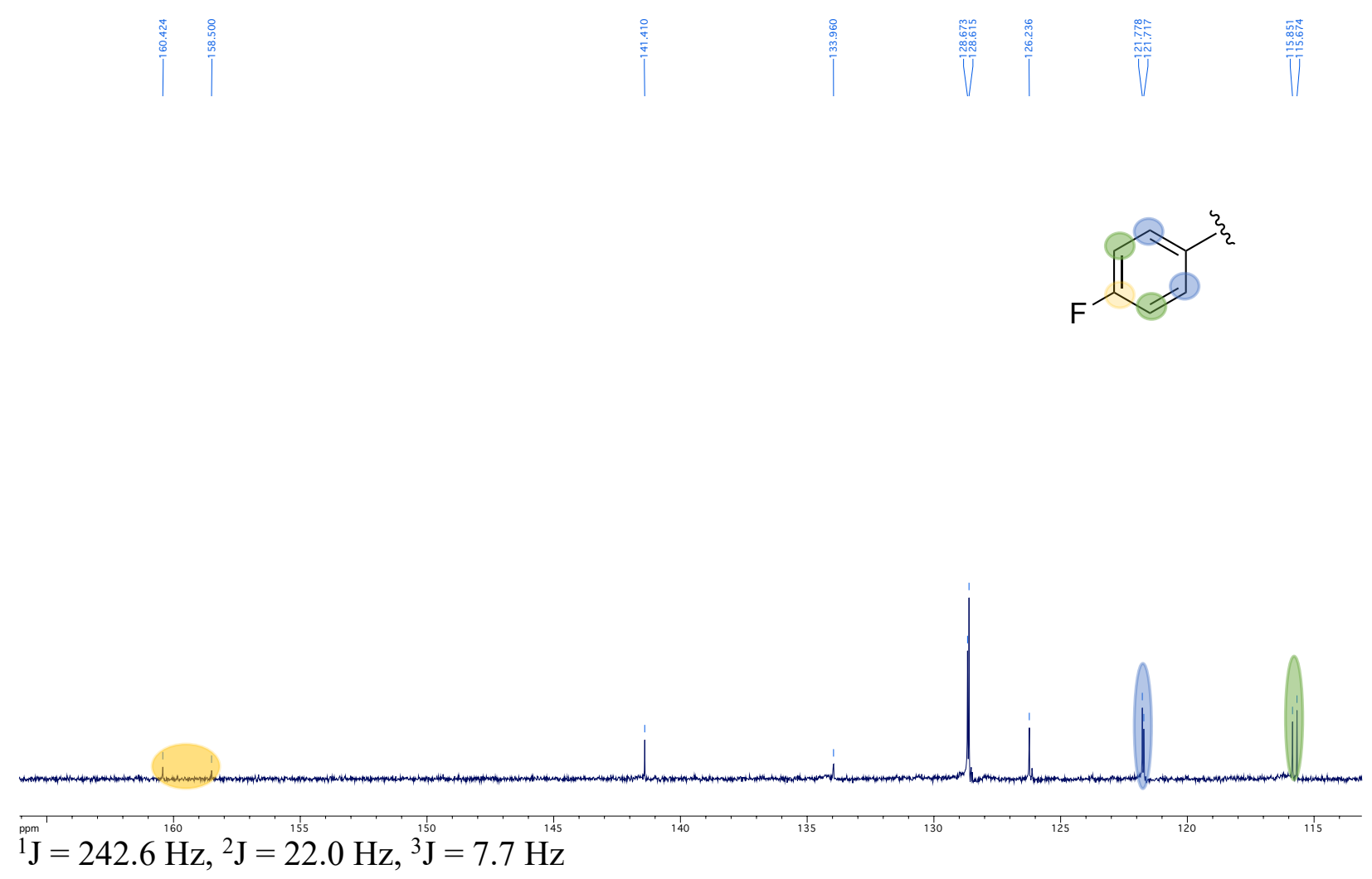

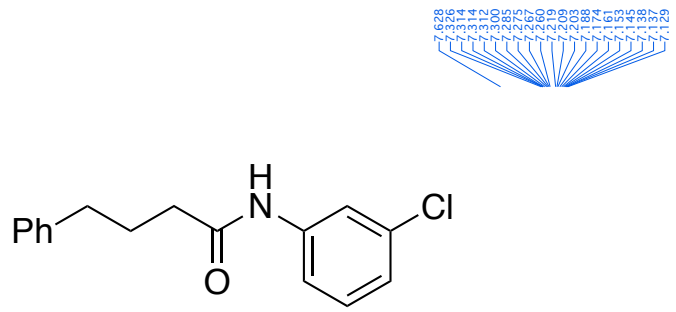

3t
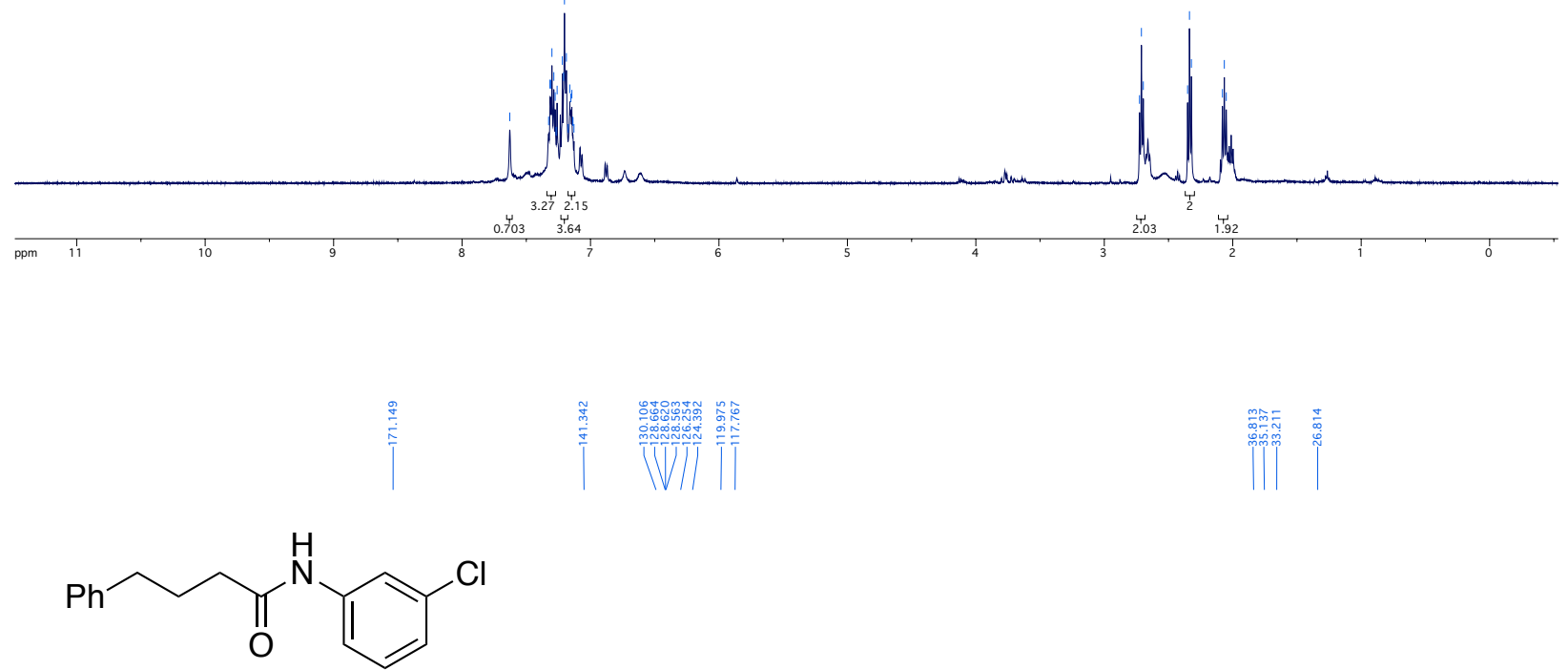

3t

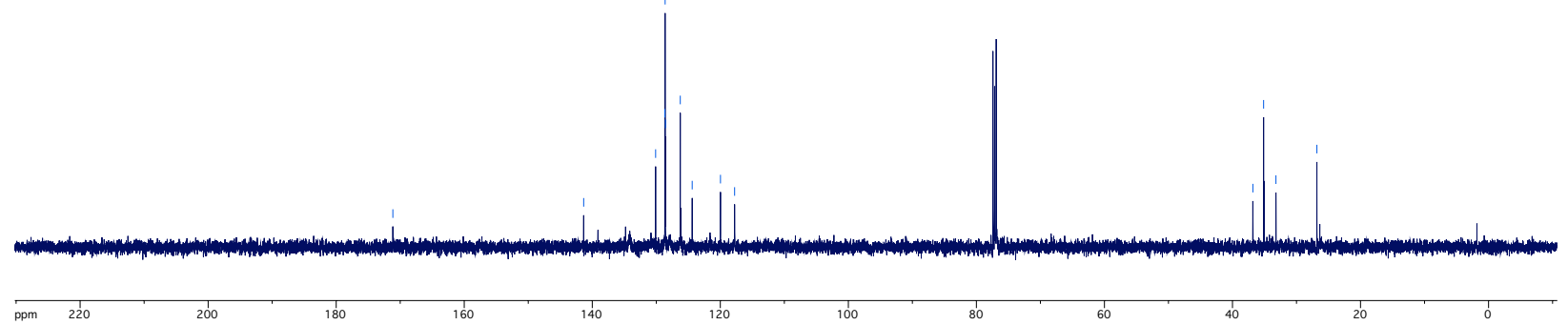



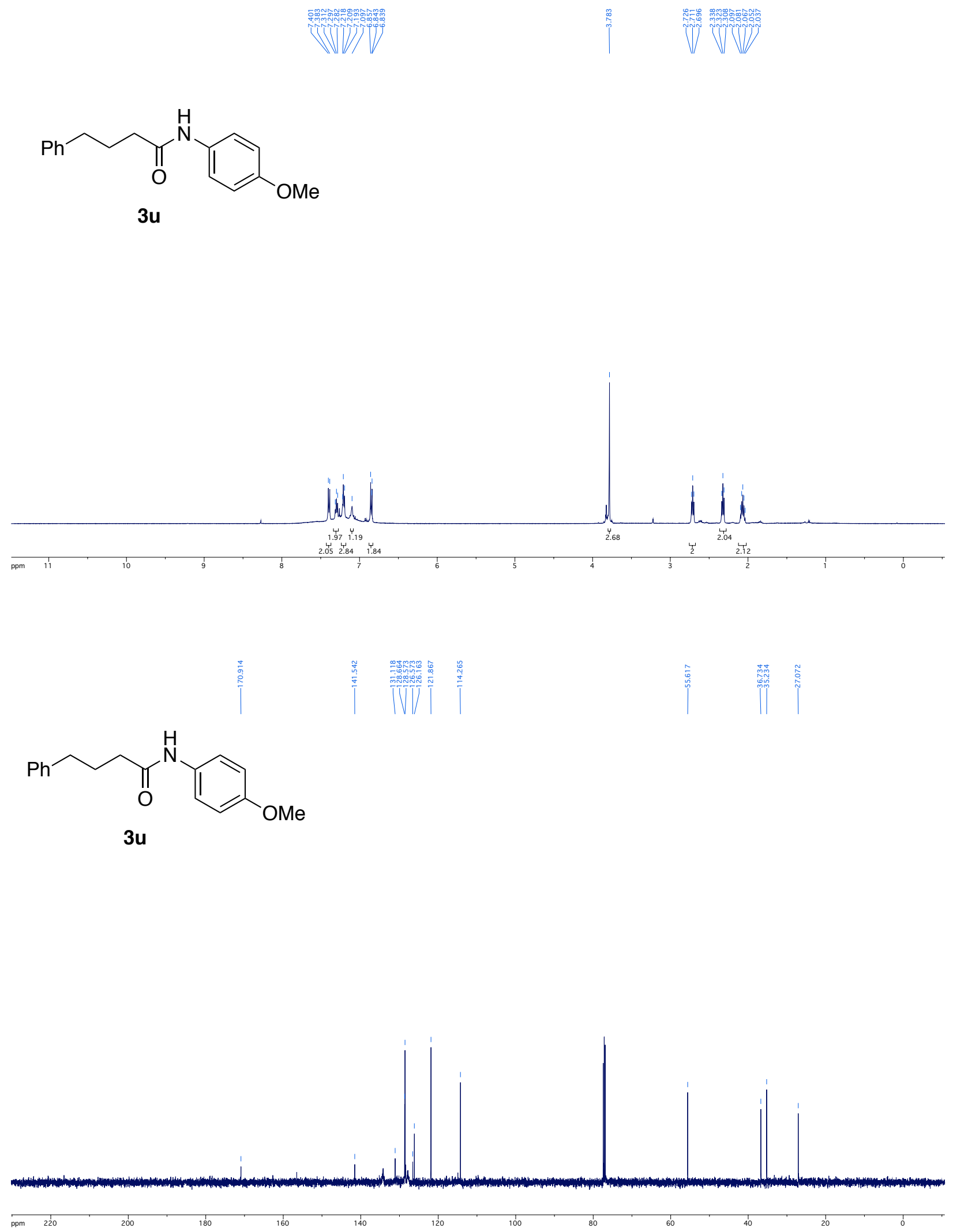

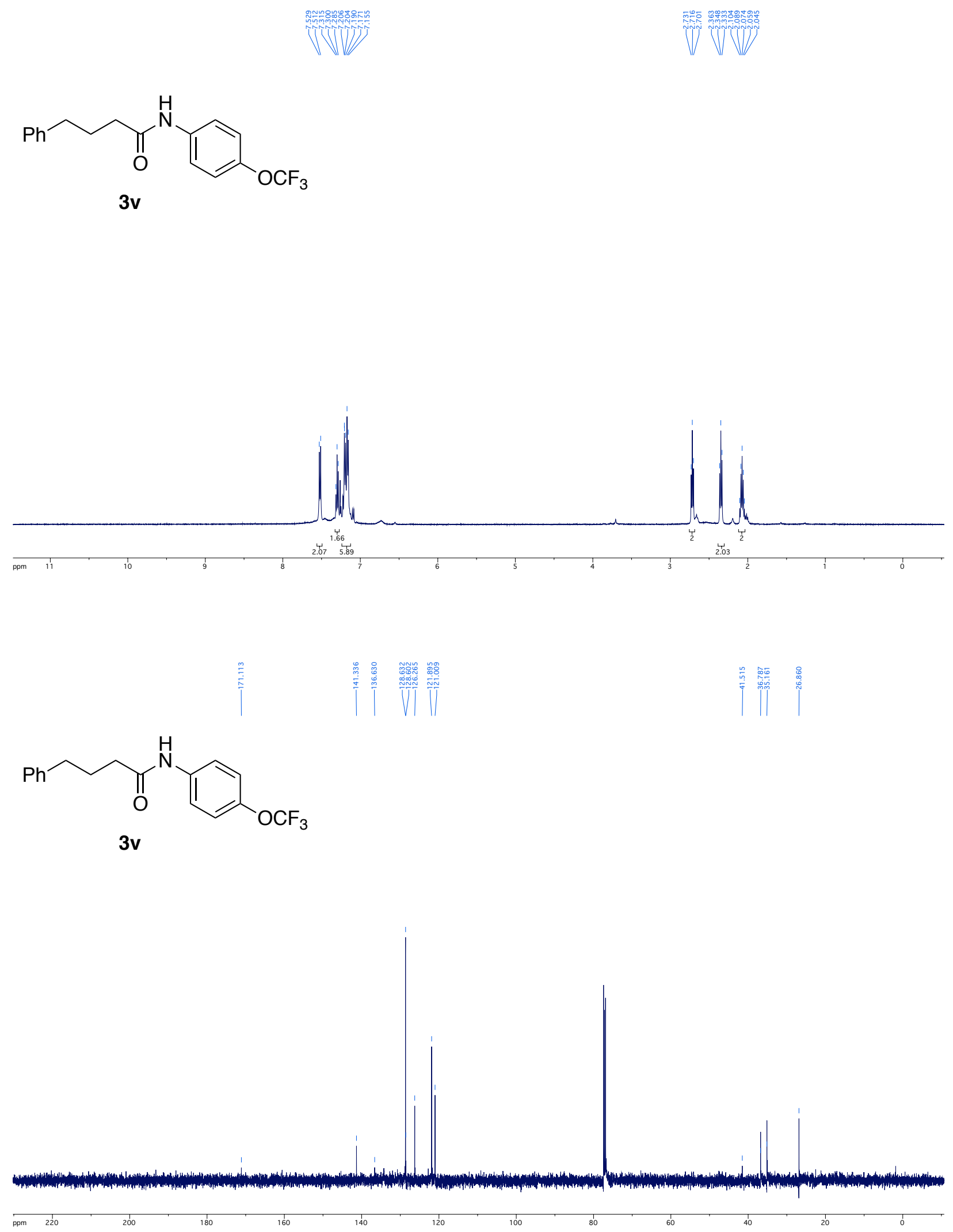


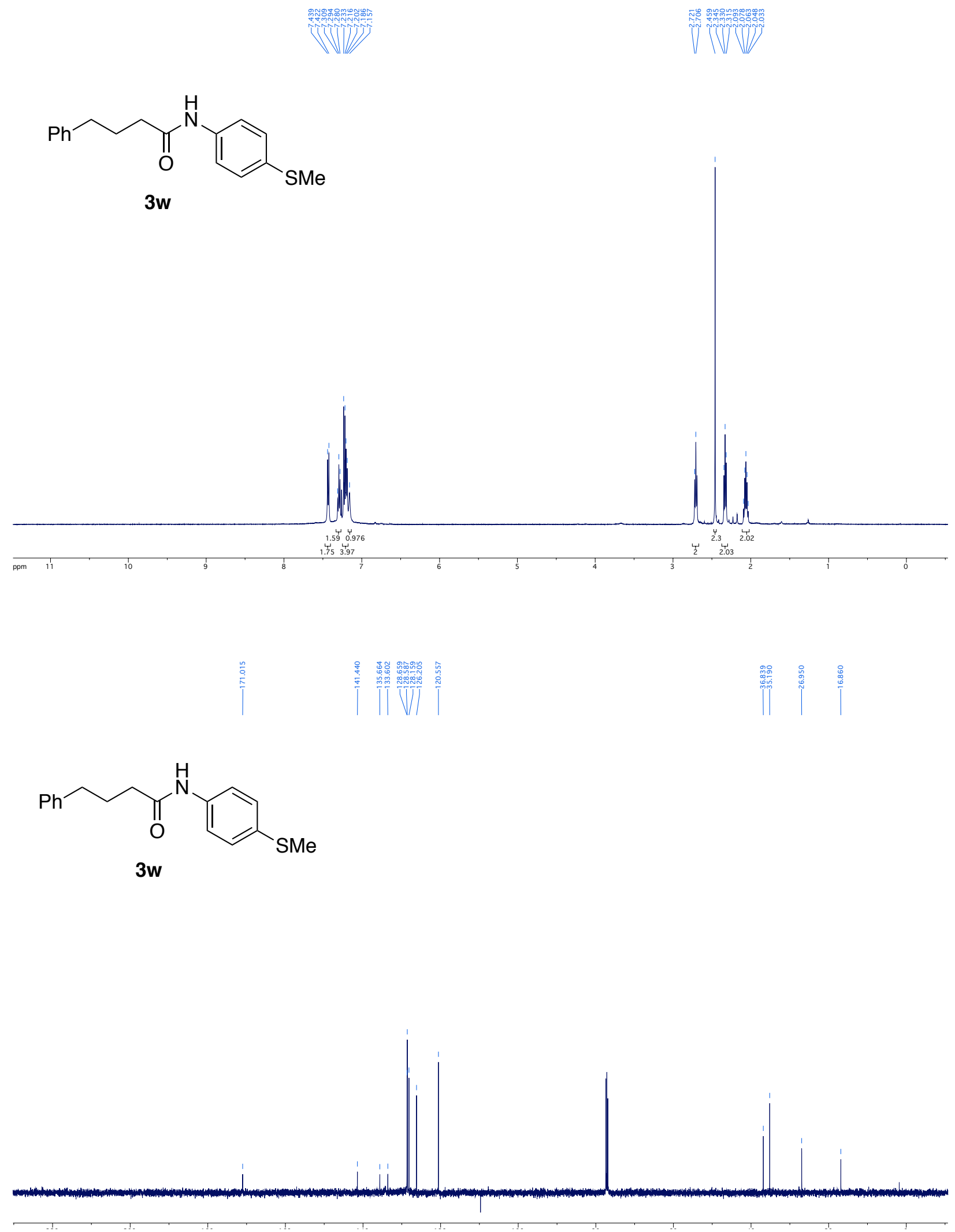



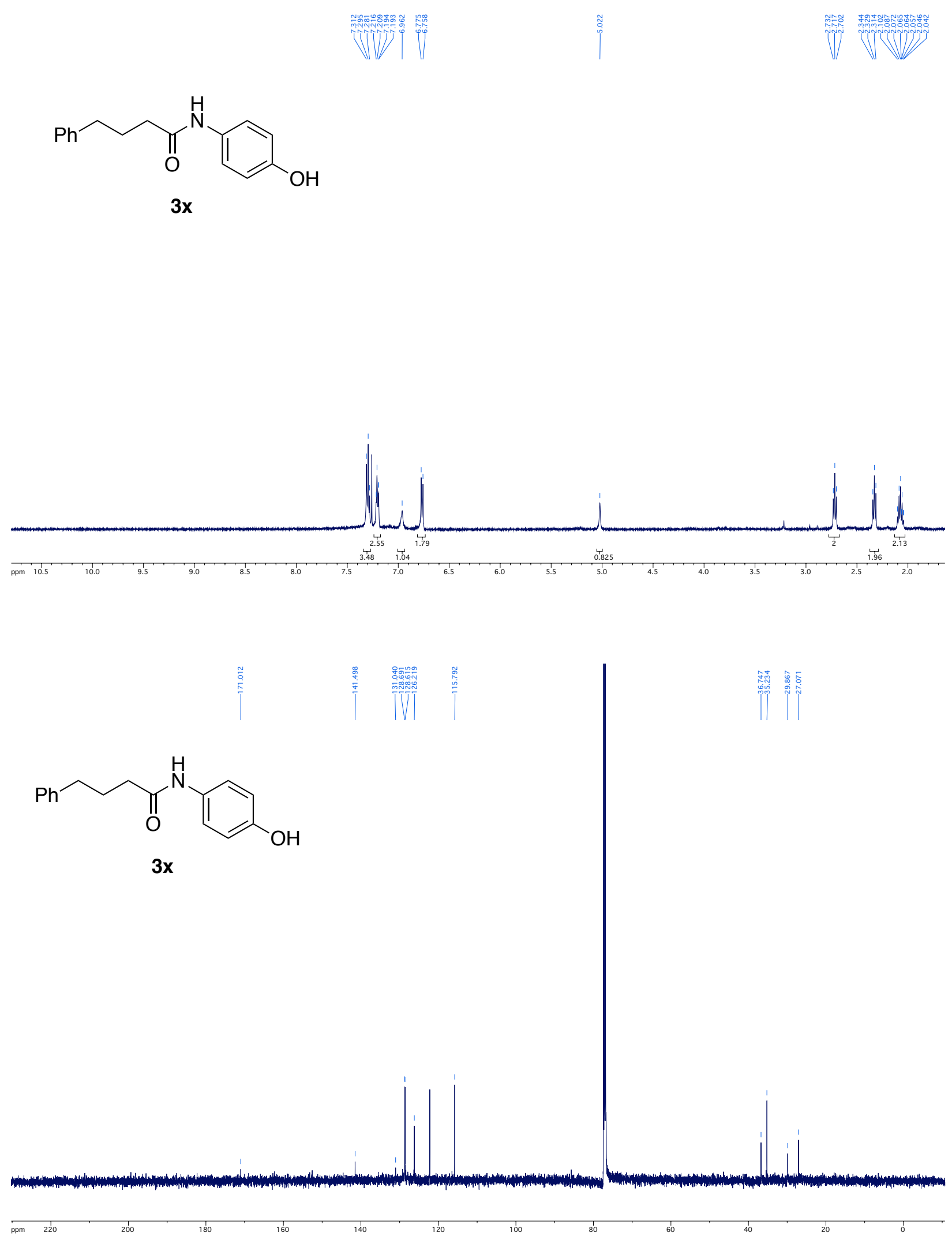


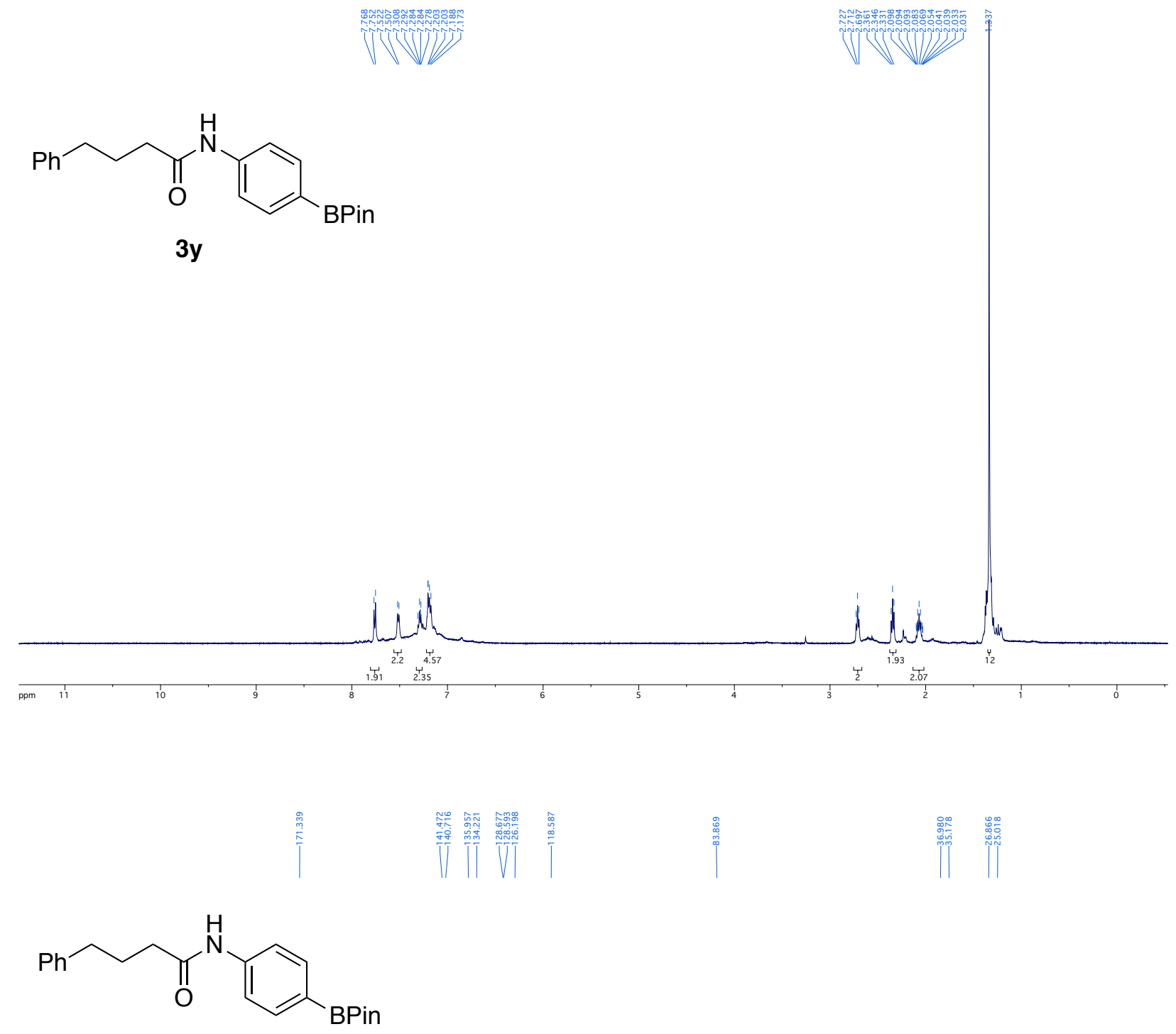

3y

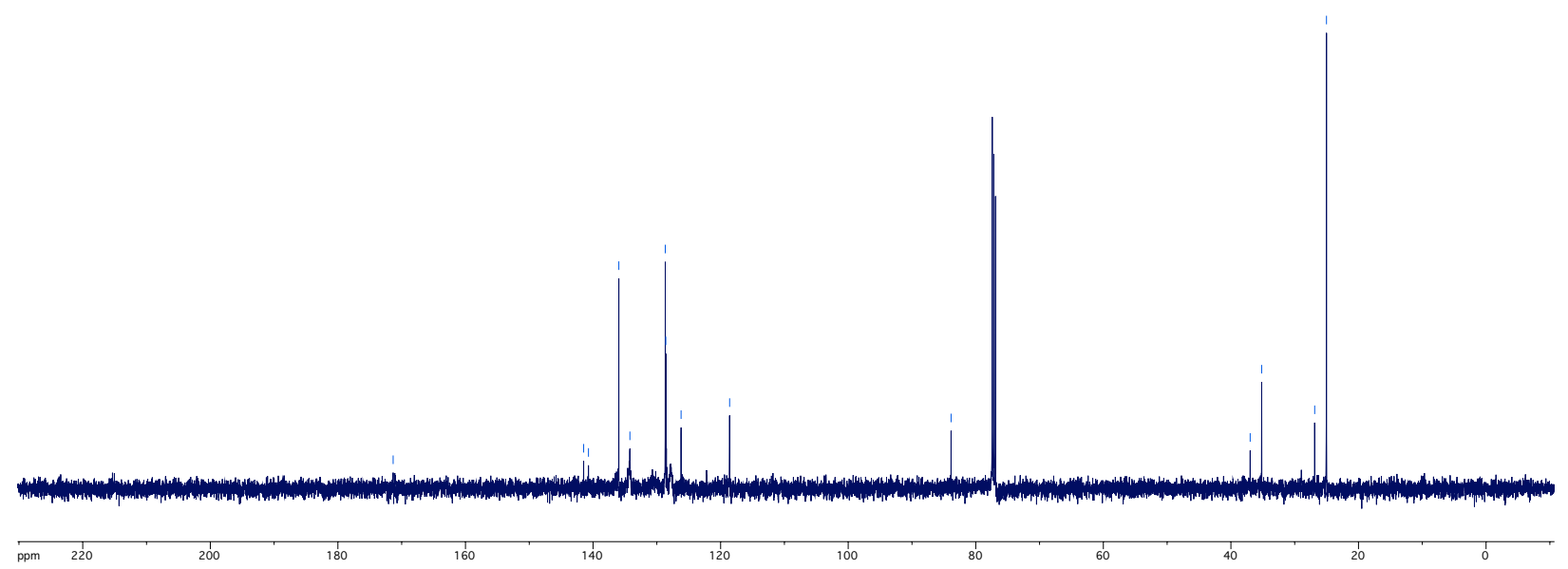




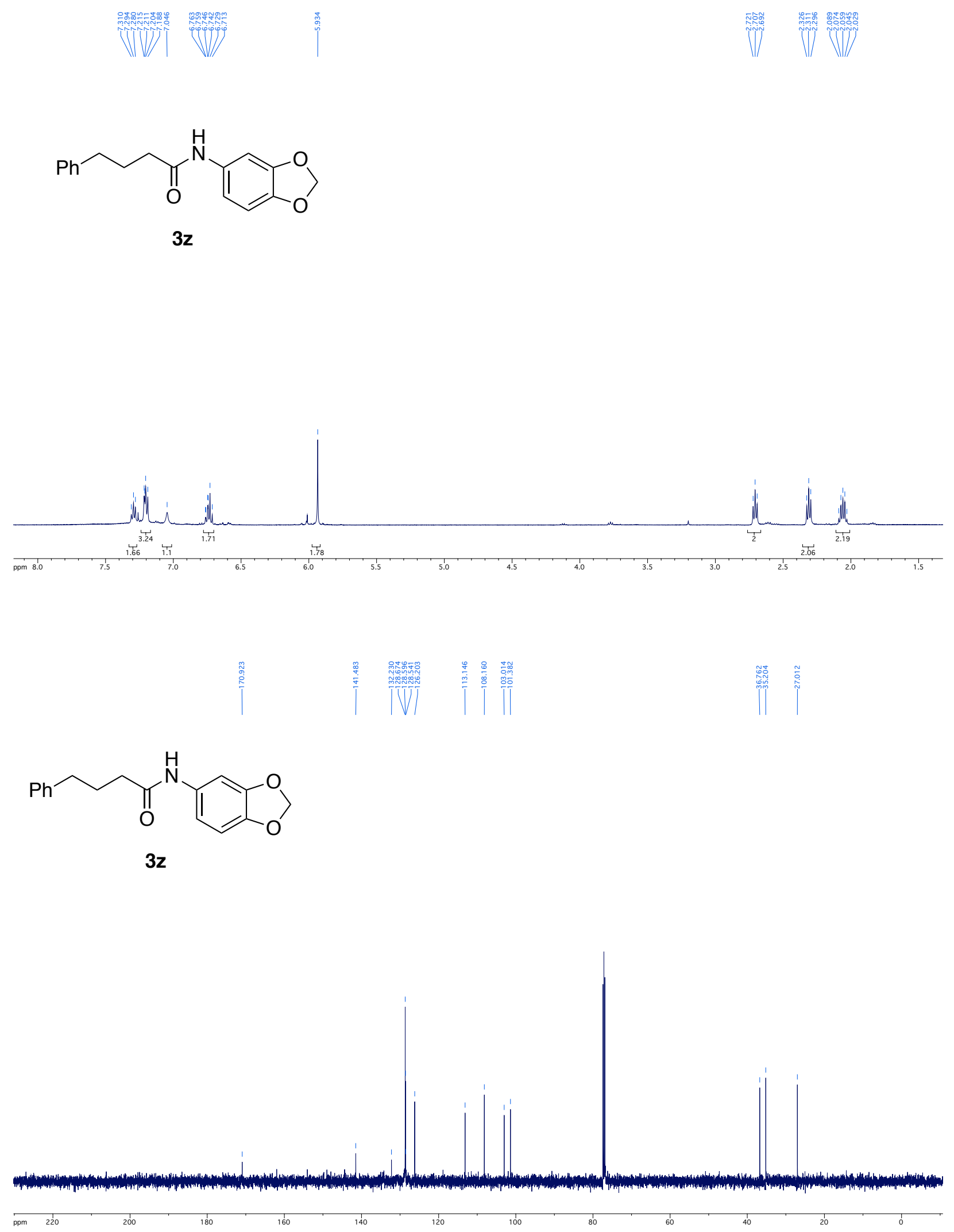




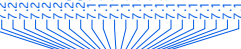

( VIII/IIJ

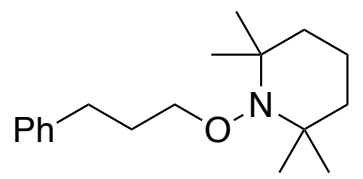

8
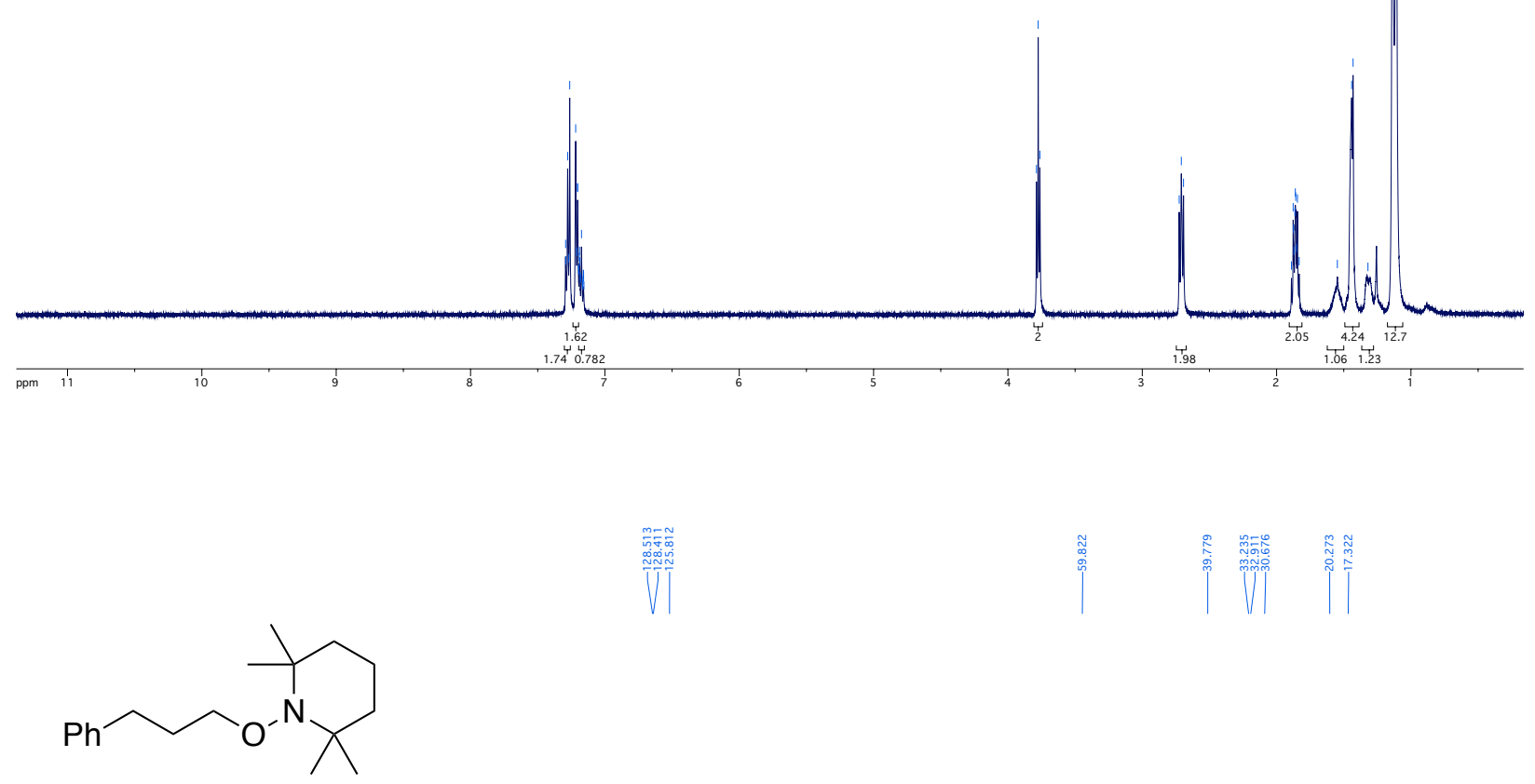

8
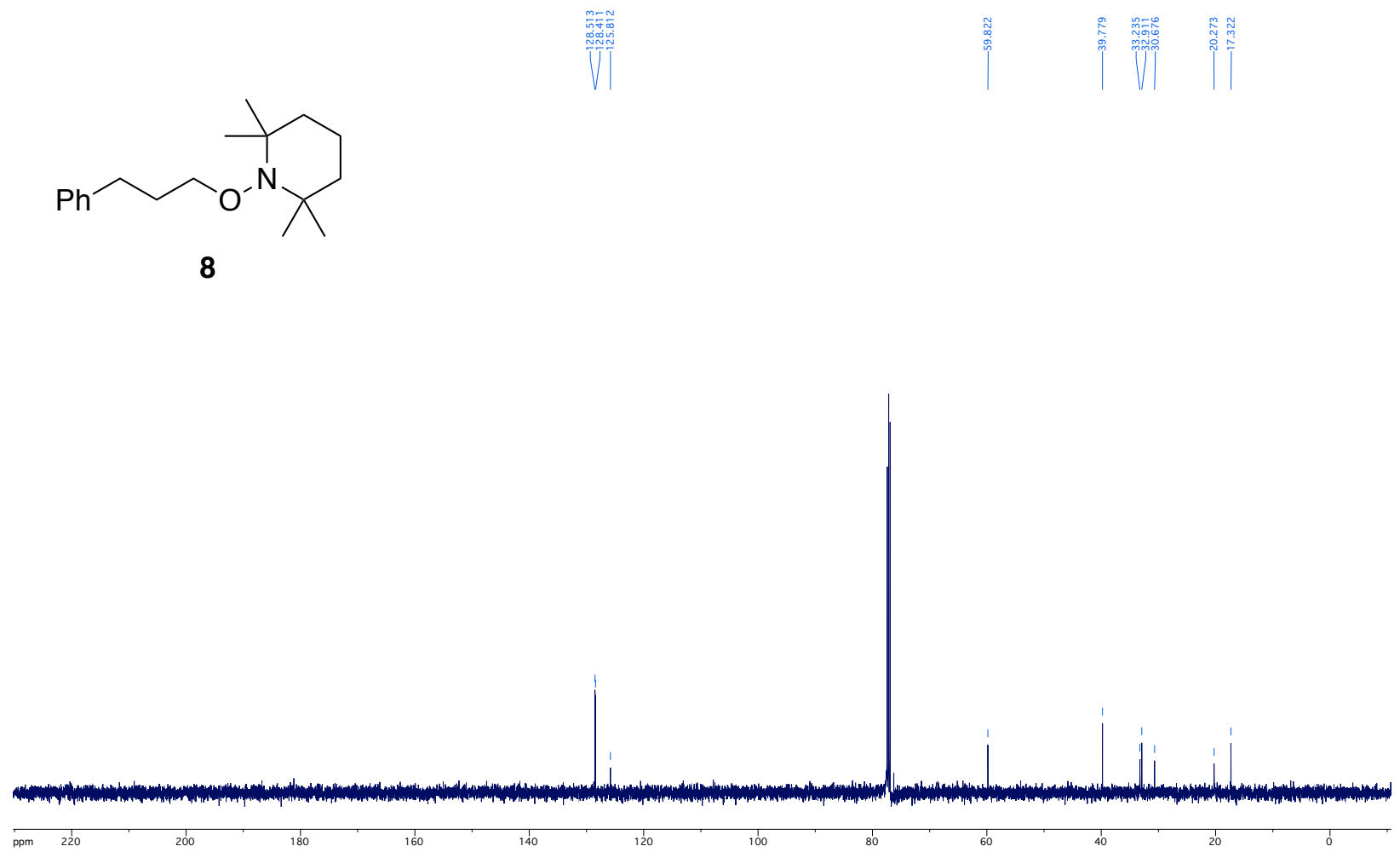


\section{References}

1 Xie, W.; Chang, S. [Cu(NHC)]-Catalyzed C-H Allylation and Alkenylation of both Electron-Deficient and Electron-Rich (Hetero)arenes with Allyl Halides. Angew.Chem., Int. Ed. 2016, 55, 1876.

${ }^{2}$ Santoro, O.; Collado, A.; Slawin, A. M. Z.; Nolan, S. P.; Cazin, C. S. J. A general synthetic route to $[\mathrm{Cu}(\mathrm{X})(\mathrm{NHC})]$ $(\mathrm{NHC}=\mathrm{N}$-heterocyclic carbene, $\mathrm{X}=\mathrm{Cl}, \mathrm{Br}$, I) complexes. Chem. Commun. 2013, 49, 10483.

${ }^{3}$ Semba, K.; Fujihara, T.; Xu, T.; Terao, J.; Tsuji, Y. Copper-Catalyzed Highly Selective Semihydrogenation of NonPolar Carbon-Carbon Multiple Bonds using a Silane and an Alcohol. Adv. Synth. Catal. 2012, 354, 1542.

${ }^{4}$ Zhao, S.; Mankad, N. P. Cu-Catalyzed Hydroxymethylation of Unactivated Alkyl Iodides with CO to Provide One Carbon Extended Alcohols. Angew. Chem., Int. Ed. 2018, 57, 5867.

${ }^{5}$ Fang, X.; Jackstell, R.; Beller, M. Selective Palladium-Catalyzed Aminocarbonylation of Olefins with Aromatic Amines and Nitroarenes. Angew. Chem., Int. Ed. 2013, 52, 4089.

${ }^{6}$ Liu, S.; Wang, H.; Dai, X.; Shi, F. Organic ligand-free carbonylation reactions with unsupported bulk Pd as catalyst. Green Chem., 2018, 20, 3457.

${ }^{7}$ Polucci, P.; Magnahi, P.; Angiolini, M.; Asa, D. et al. Alkylsulfanyl-1,2,4-triazoles, a New Class of Allosteric Valosine Containing Protein Inhibitors. Synthesis and Structure-Activity Relationships. J. Med. Chem. 2013, 56, 437.

${ }^{8}$ Hernandz, E.; Hoberg, H. Stoichiometric and catalytic carbon-carbon coupling between 1,3-butadiene and phenyl isocyanate in nickel(0) complexes. J. Organomet. Chem. 1987, 327, 429. 